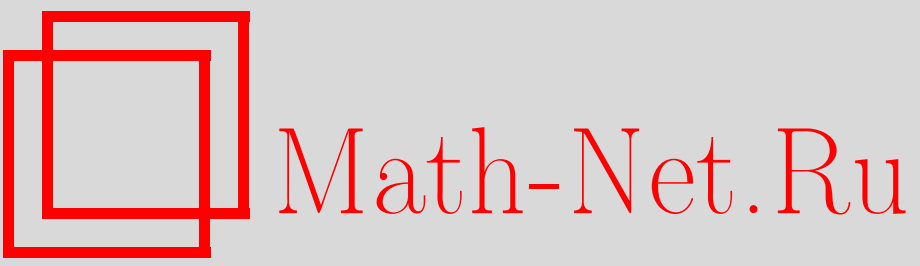

А. Ю. Китаев, Квантовые вычисления: алгоритмы и исправление ошибок, УМН, 1997, том 52, выпуск 6, 53-112

DOI: https://doi.org/10.4213/rm892

Использование Общероссийского математического портала Math-Net.Ru подразумевает, что вы прочитали и согласны с пользовательским соглашением

http://www.mathnet.ru/rus/agreement

Параметры загрузки:

IP : 54.92 .164 .108

26 апреля 2023 г., 10:45:29 


\section{КВАНТОВЫЕ ВЫЧИСЛЕНИЯ: АЛГОРИТМЫ И ИСПРАВЛЕНИЕ ОШИБОК}

А. Ю. КИТАЕВ

СОДЕРЖАНИЕ

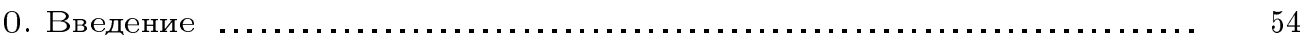

1. Абелева задача о стабилизаторе ............................... 57

2. Классические модели вычислений ................................ 59

2.1. Булевы схемы и последовательности операций ................... 59

2.2. Обратимше вычисления ................................... 61

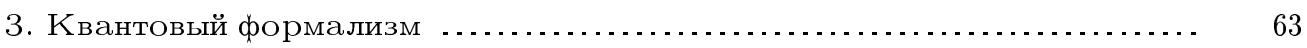

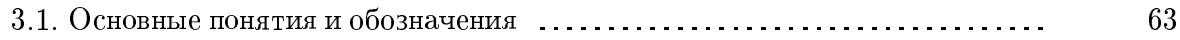

3.2. Преобразования смешанных состояний …..................... 67

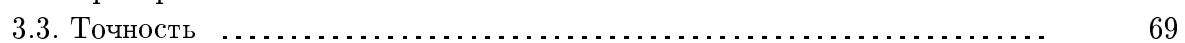

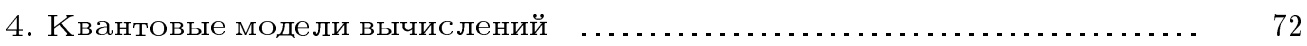

4.1. Определения и основные свойства . .......................... 72

4.2. Построение различных операторов из элементов базиса . . . . . . . . . . $\quad 74$

4.3. Обобщенное квантовое управление и универсальные схемы ...........

5. Измеряющие операторы ....................................... 80

6. Полиномиальный квантовый алгоритм для задачи о стабилизаторе ... 82

7. Вычисления с возмущениями: выбор модели ....................... 84

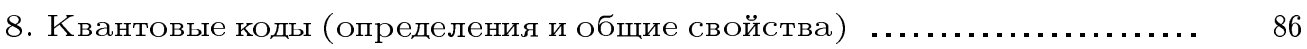

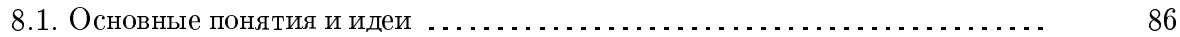

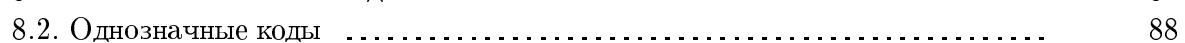

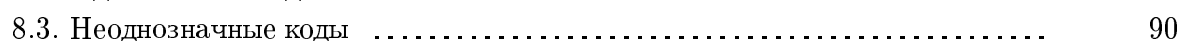

9. Симплектические (аддитивные) коды …........................ 92

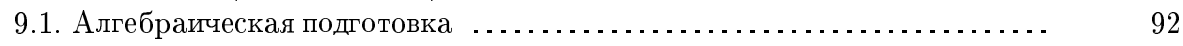

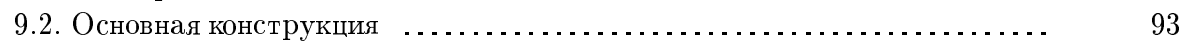

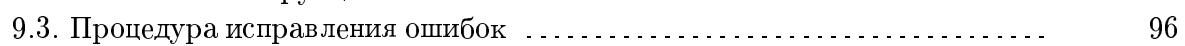

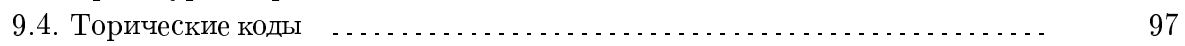

10. Исправление ошибок в процессе вычисления: общие принципы ...... 98

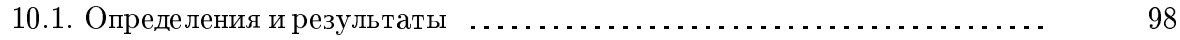

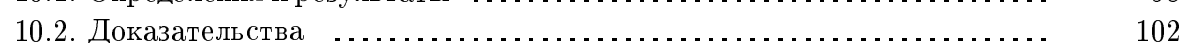

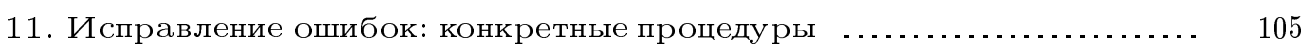

11.1. Симплекто-классический случай . . . . . . . . . . . . . . . . . . . . . . . 105

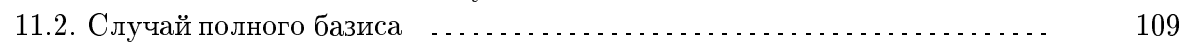

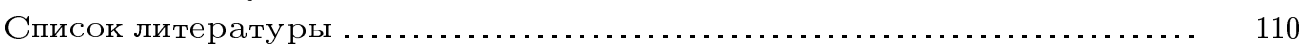

(C) А. Ю. КитАев 1997 
Первая часть статьи (разделы 1-6) подчинена, в основном, одной цели - показать вычислительную мошь квантового компьютера на примере задачи о стабилизаторе в групше $\mathbf{Z}^{k}$. K этой задаче сводятся дискретный логарифм и разложение на простые множители. Формальное определение квантового компютера (точнее, квантовой схемы) появляется в разделе 4. Все необходимые сведения из теории вычислений и квантовой механики содержатся в разделах 2 и 3.

Чтение второй части можно начать с теории однозначных квантовых кодов (разделы 8.1, 8.2 и 9). Эта теория достаточно замкнута, проста и содержательна сама по себе. Однако в настояшей статье она рассматривается как средство для решения проблемы возмущений - построения квантового компютера из ненадежных (неточных, подверженных возмущениям) Элементов. Этой теме посвящены разделы $7,8.3$, 10 и 11. Содержательные результаты получены в разделах 10.1 и 11; все остальное довольно утомительная техническая подготовка. (До сих пор данная проблема рассматривалась лишш на физическом уровне строгости. Формальный подход выявил одно тонкое место, где наивные рассуждения могут привести к ошибке - см. раздел 10.1.) Изначальная постановка проблемы нечеткая и опирается на интуитивное представление о том, как могут работать реальные вычислительные устройства. Это представление формализовано в разделе 7 , но окончательная формулировка задачи (так называемая полиномиальная система исправления ошибок, см. определение 10.4) появляется лиш в середине раздела 10.1 .

\section{0. Введение}

Строение атомов, химическая связь в молекулах, разнообразные физические свойства твердых тел - все это, в принципе, вытекает из фундаментальных законов квантовой механики, открытых 70 лет назад. Многие из указанных явлений поняты и поддаются численным расчетам; физики-теоретики придумали различные приближенные методы решения квантовомеханических задач. Однако в общем случае моделирование квантовых процессов требует неприемлемо большого объема вычислений.

Лишь недавно стало ясно, что эта трудность имеет и обратную, положительную сторону. Если нельзя (за приемлемое время) моделировать квантовую механику на обычном компьютере, то можно попытаться использовать какую-нибудь квантовую систему для решения трудных вычислительных задач. Прежде всего, конечно, нужно сформулировать простую и достаточно общую математическую модель квантовых вычислений. Эта идея, по-видимому, впервые была высказана Ю.И. Маниным в книге [1]. Ряд авторов показали, как проводить обычные вычисления на квантовых устройствах [2]-[4]. Но только в 1985 году Deutsch дал общее определение так назьваемой квантовой машины Тьюринга [5]. В 1989 году он сфформулировал другую, более удобную модель - квантовые схемы [6]. Эквивалентность этих двух моделей доказал Yао [7].

Вычислительную задачу принято считать трудной, если для нее не сушествует полиномиального алгоритма [8]. Алгоритм назьвается полиномиальным, если число шагов вычисления растет не быстрее, чем некоторая степень длины входа задачи. Очевидно, что это определение зависит от используемой модели вычислений. Оказывается, что большинство абстрактных моделей вьчислений (машина Тьюринга, машина с прямым доступом к памяти (RAM) и др.) полиномиально эквивалентны друг другу. Иными словами, одна машина может моделировать другую, в худшем случае, с поли- 
номиальным замедлением. Например, если какое-либо вычисление требует $t$ шагов на машине с прямым доступом к памяти, то его можно выполнить за $O\left(t^{2}\right)$ шагов на машине Тьюринга.

Заметим, что упомянутые вычислительные модели могут быть реализованы физически. (Практически любой из сушествуюших в настоящее время компьютеров можно рассматривать как реализацию RAM.) Обратно, классическую механику можно моделировать на компютере за полиномиальное по числу степеней свободы время. Однако с квантовой механикой дело обстоит иначе. Рассмотрим систему с двумя базисными состояниями, $|0\rangle$ и $|1\rangle$. Такая система, независимо от ее физической реализации, назьвается квантовым битом, или кубитом. Общее квантовое состояние кубита есть суперпозиция (линейная комбинация) $c_{0}|0\rangle+c_{1}|1\rangle$ с комплексными коэффициентами $c_{0}, c_{1}$. Система из $n$ кубитов имеет $2^{n}$ базисных состояний; общее состояние есть вектор в $2^{n}$-мерном комплексном пространстве. Преобразование (эволюция) состояния за определенньй промежуток времени описьвается унитарной матрицей размера $2^{n} \times 2^{n}$. Естественньй способ моделирования состоит в умножении таких матриц, что требует экспоненциального объема вычислений.

Квантовое вычисление - это последовательность элементарных унитарных преобразований (операций), каждое из которых затрагивает небольшое число кубитов. При этом входное слово кодируется базисньм вектором, а результат вычисления определяется вероятностно при помощи операторов проектирования (см. определение 4.1). В этой новой вычислительной модели любую квантовую систему можно моделировать за полиномиальное время. В принципе, квантовьй компьютер можно реализовать физически, однако вряд ли это будет сделано в ближайшее время. (По поводу некоторых экспериментальных успехов в этом направлении см. [9], [10].) Настояшая статья посвящена двум обшим проблемам, не зависяшим от конкретной физической реализации:

1) Может ли квантовый компьютер решать “классические” трудные задачи (из области комбинаторики, теории чисел и т. д.)?

2) В какой степени квантовьй компьютер устойчив к возмущениям и неточности реализации элементов? Можно ли организовать процесс вычисления так, чтобы умеренное возмущение не влияло на результат?

В 1994 году Shor придумал полиномиальные квантовые алгоритмы для дискретного логарифма и разложения целого числа на простые множители [11]. Обе задачи считаются трудными, ${ }^{1}$ и это предположение положено в основу так назьваемых криптосистем с открытым ключом [12]. (Таким образом, при помоши квантового компьютера можно “взламьвать” соответствуюшие шифры.) Однако эффективного квантового алгоритма для NP-полной задачи пока не найдено.

Напомним определение дискретного логарифма. Пусть $p$ - простое число, $\zeta$ - первообразньй корень в поле вычетов по модулю $p$. Тогда отображение $x \mapsto \zeta^{x}$ является изоморфизмом аддитивной групш вычетов по модулю $p-1$ в мультипликативную группу вычетов по модулю $p$. Обратное отображение назьвается дискретным логарифмом.

Мы опишем полиномиальный квантовьй алгоритм для гораздо более общей задачи - нахождение стабилизатора заданного элемента относительно действия абелевой

\footnotetext{
${ }^{1}$ Ни про одну естественную вьчислителшную задачу пока не доказано, что она является трудной. Однако есть немало задач, для которых полиномиальных алгоритмов найти не удалось.
} 
групшы $G$ на конечном множестве. Случай $G=\left(\mathbf{Z}_{p}\right)^{k}$ был рассмотрен Д. Григорьевым [14]; в общем виде задача решена в работе автора [15]. Ключевым моментом является процедура измерения собственного значения унитарного оператора (см. раздел 5). Используется также метод Simon'a [13] для порождения случайного элемента групшы характеров.

Проблема возмушений имеет первостепенное значение для практической реализации квантового компьютера. В общем случае, последовательность из $L$ операций дает правильньй результат с вероятностью $\geqslant 1-\varepsilon$, если каждая операция выполнена с точностью $\varepsilon /(2 L)$ (см. предложение 4.2 или статью [16]). В реальных условиях возмущение будет действовать на кубиты, даже если с ними ничего не делать. В результате квантовое состояние будет портиться. Такая ситуация не слишком обнадеживает. Было бы желательно уметь сохранять квантовое состояние и проводить сколь угодно длинные квантовые вычисления с операциями фиксированной точности. Другими словами, нужно научиться моделировать точные операции неточными.

Для классических вычислений проблема возмушений давно решена. Простейший метод повьшения надежности заключается в том, чтобы хранить несколько копий каждого бита, периодически их сверяя. К сожалению, этот метод неприменим в квантовом случае, поскольку квантовое состояние невозможно скопировать (оператор копирования $|\xi\rangle \mapsto|\xi\rangle \otimes|\xi\rangle$ не является линейным).

Более обший способ борьбы с возмушениями основан на кодах, исправляюших ошибки. Классическая теория таких кодов весьма обширна [17]. Простейший классический код $\operatorname{REP}(n)$ основан на повторении: 0 представляется словом из $n$ нулей, а 1 представляется словом из $n$ единиц. Квантовым кодом типа $(n, m)$ называется унитарное вложение пространства состояний $m$ кубитов в пространство состояний $n$ кубитов. Первьй пример квантового кода, исправляюшего ошибки, придумал Shor [18]. Вскоре Calderbank и Shor [19], а также Steane [20] предложили довольно обший метод построения квантовых кодов. (В статье [19] доказано также сушествование так называемых "хороших" квантовых кодов). Различные примеры квантовых кодов содержатся в работах [21]-[28]. Наконец, Calderbank, Rains, Shor и Sloan [29] определили очень широкий и естественный класс квантовых кодов, включаюший в себя все ранее известные примеры. Мы будем называть эти коды симплектическими, покольку они связаны с симплектической группой над полем $\mathbf{F}_{2}$; более традиционное название - аддитивные коды. Пример неаддитивного кода построен в статье [30].

В настоящей статье не рассматриваются такие вопросы как пропускная способность квантового канала [31]-[34], оптимальность квантового кодирования [19], [25], [35], [36], энумераторы квантовых кодов [37], [38], использование "запутанных" состояний (entangled states) и "квантовая телепортация" [39]-[41]. Мы ограничимся всего двумя далеко не оптимальными, но интересньми примерами. В разделе 9.4 построена бесконечная последовательность так назьваемых торических кодов $\operatorname{TOR}(k)$ [42]. Важное свойство этих кодов состоит в том, что они являются кодами с локальньми проверками.

Подчеркнем, что мы собираемся использовать коды не для передачи информации (или квантовых состояний), а для борьбы с ошибками в процессе вычисления. Это диктует весьма специфические требования к самим кодам и к связанньм с ними вычислительньм процедурам. В частности, процедура исправления ошибок должна быть устойчива к новьм ошибкам, которые могут случиться в процессе ее вьполнения. 
Кроме того, нужно научиться производить операции с закодированными кубитами, не раскодируя их явно (иначе они окажутся не зашищенными от возмущений). Далеко не любые коды позволяют это делать.

Принципиальное решение проблемы возмушений для квантовых вычислений получено Shor'oм [43]. Он показал, что вычисление длины $L$ может быть вьполнено при точности каждой операции $(\log L)^{-c}(c=$ const). В настоящей статье этот результат усилен. Мы покажем, что достаточно фиксированной точности. Для этого используются так называемые каскадные коды. Аналогичные (но технически отличные) конструкции были предложены в работах [44]-[46]. Другие результаты на эту тему содержатся в статьях [47], [48].

\section{1. Абелева задача о стабилизаторе}

Пусть $G$ - группа, действующая на конечном множестве $M$. Предположим, что это действие и групповые операщии в $G$ легко вычислимы. Вычислить стабилизатор заданного элемента $a \in M$. Эта задача (которую еше предстоит уточнить) включает в себя много интересных случаев, например, изоморфизм графов. К сожалению, пока мы не в состоянии решить задачу во всей ее обшности. Вместо этого мы будем предполагать, что групп $G$ абелева. Поскольку любая конечно-порожденная абелева группа является фактор-группой $\mathbf{Z}^{k}$, мы можем без ограничения обшности положить $G=\mathbf{Z}^{k}$. Менее общий случай $G=\left(\mathbf{Z}_{p}\right)^{k}$ был изучен Д. Григорьевым [14]: в этой статье построен алгоритм, полиномиальньй по $k$ и $p$ (но не по $\log p)$. При тех же предположениях мы получим полностью полиномиальный алгоритм, т.е. алгоритм, полиномиальный по $k+\log p$.

Чтобы сформулировать задачу как вычислительную, мы будем считать, что множество $M$ отождествлено с некоторым подмножеством булева куба $\mathbf{B}^{n}=\{0,1\}^{n} .^{2}$ Каждьй элемент $g=\left(m_{1}, \ldots, m_{k}\right) \in \mathbf{Z}^{k}$ можно представить двоичньм словом ${ }^{3}$ длины $\operatorname{size}(g)=\sum_{i=1}^{k} O\left(\log \left(\left|m_{i}\right|+1\right)\right)$.

Перейдем к точной формулировке. Абелева задача о стабилизаторе (точнее, представитель этой задачи) определяется заданием следуюших объектов:

- два положительных целых числа, $k$ и $n$. Число $k+n$ назьвается размером задачи;

- элемент $a \in \mathbf{B}^{n}$;

- функция $F: \mathbf{Z}^{k} \times M \rightarrow M\left(a \in M \subseteq \mathbf{B}^{n}\right)$ такая, что

$F(0, x)=x, \quad F(g+h, x)=F(g, F(h, x))$ для любых $g, h \in \mathbf{Z}^{k}, \quad x \in M$.

Функцию $F$ следует рассматривать как оракул, т.е. "черный яшик", которьй получает входное слово $(g, x) \in \mathbf{Z}^{k} \times \mathbf{B}^{n}$ и сразу же выдает ответ $y \in \mathbf{B}^{n}$ такой, что $y=F(g, x)$ для любых $g \in \mathbf{Z}^{k}, x \in M$. (Если $x \notin M$, то оракул может не дать никакого ответа или выдать произвольный ответ.) Предполагается, что к оракулу $F$

\footnotetext{
${ }^{2}$ Можно было бы рассмотреть столь же естественную, но более общую ситуацию: каждый элемент $M$ имеет несколько представлений в $\mathbf{B}^{n}$, причем эквивалентность этих представлений проверяется при помощи некоторой эффективной процедуры. К сожалению, наш метод не работает в этом случае.

${ }^{3}$ Конечно, это представление не единственно. Однако, в различных "естественных" представлениях $\operatorname{size}(g)$ может отличаться не более, чем на постоянный множитель.
} 
можно обрашаться в процессе классического или квантового вычисления, см. разделы 2 и 4.1. Постановка задачи не включает в себя явного описания множества $M$; достаточно знать, что такое множество сушествует.

ЗАмечАнИЕ. Во всех осмысленных приложениях (см. примеры ниже) функция $F$ вычислима за полиномиальное время. Квантовый компьютер может сам выполнить эту работу, так что нет нужды обрашаться к оракулу. Опишем эту ситуацию в более строгих терминах. Пусть poly обозначает любую функцию, которая растет не быстpee, чем полином, т.е. $\operatorname{poly}(x)=x^{O(1)}$. Предположим, что функция $(g, x) \mapsto F(g, x)$ вьгислима на некоторой машине Тьюринга за время poly $(\operatorname{size}(g)+n)$. При фиксированной функции полиномиального роста poly это условие определяет ограниченную абелеву задачу о стабилизаторе, т.е. некоторьй подкласс представителей этой задачи. В этом случае можно построить полиномиальньй квантовьй алгоритм, на вход которого подается описание машины Тьюринга, вьчисляющей $F$.

Стабилизатором $а$ относительно $F$ назьвается множество $\operatorname{St}_{F}(a)=\left\{g \in \mathbf{Z}^{k}\right.$ : $F(g, a)=a\}$. Это подгруппа конечного индекса $\leqslant|M| \leqslant 2^{n}$ в $\mathbf{Z}^{k}$. Следовательно, стабилизатор $\operatorname{St}_{F}(a)$ изоморфен $\mathbf{Z}^{k}$ и имеет базис $\left\{g_{1}, \ldots, g_{k}\right\}$ полиномиального размера, т.е. $\sum_{j=1}^{k} \operatorname{size}\left(g_{j}\right) \leqslant \operatorname{poly}(n+k)$. Любой такой базис допустим в качестве решения задачи о стабилизаторе. Сушествует эффективная процедура, позволяющая проверить, являются ли два множества $\left\{g_{1}, \ldots, g_{k}\right\}$ и $\left\{g_{1}^{\prime}, \ldots, g_{k}^{\prime}\right\}$ базисами одной и той же подгруппы ранга $k$ в $\mathbf{Z}^{k}$. Более того, существует довольно простой полиномиальный алгоритм приведения базиса к единственной (для данной подгруппы $\left.A \subseteq \mathbf{Z}^{k}\right)$ канонической форме. Канонический базис состоит из столбцов матрицы $\left(m_{i j}, i, j=1, \ldots, k\right)$, которая однозначно характеризуется следуюшими условиями:

$$
\begin{aligned}
m_{i j} & =0, \quad \text { если } i>j, \\
m_{i i} & >0, \\
0 \leqslant m_{i j} & <m_{i i}, \text { если } i<j .
\end{aligned}
$$

Таким образом, построение произвольного базиса (полиномиального размера) эквивалентно построению канонического базиса.

Вычислительные задачи "разложение на множители" и "дискретньй логарифм" сводятся к абелевой задаче о стабилизаторе. Пусть $M$ - кольцо целых чисел по модулю $q$, а $G$ - группа обратимых элементов в $M$. Если $g_{1}, \ldots, g_{k} \in G$, то можно определить действие $\mathbf{Z}^{k}$ на $M$ следуюшим образом: $F_{g_{1}, \ldots, g_{k}}:\left(m_{1}, \ldots, m_{k}, x\right) \mapsto$ $g_{1}^{m_{1}}, \ldots, g_{k}^{m_{k}} x\left(m_{i} \in \mathbf{Z}, x \in M\right)$. Рассмотрим два случая.

1. Разложение целого числа на простые множители. Пусть $k=1$. Стабилизатор элемента $1 \in M$ относительно $F_{g}$ дает порядок элемента $g$ в групе $G$. Сушествует рандомизированная редукция задачи "разложение на множители" к задаче "порядок элемента" [49]. (Краткое изложение этой редукции содержится в статье Shor'a [11].)

2. Дискретный логарифм. Пусть $q$ - простое число; тогда группа $G$ циклическая. Зафиксируем некоторый примитивньй элемент $\zeta \in G \cong \mathbf{Z}_{q-1}$. Возьмем также произвольньй элемент $g \in G$. Стабилизатором 1 относительно $F_{\zeta, g}$ является множество $P=\left\{(m, r) \in \mathbf{Z}^{2}: \zeta^{m} g^{r}=1\right\}$. Зная базис подгруппы $P \subseteq \mathbf{Z}^{2}$, легко найти элемент вида $(m,-1) \in P$. Тогда $\zeta^{m}=g$, т.е. $m$ есть дискретньй логарифм $g$ по основанию $\zeta$. 


\section{2. Классические модели вычислений}

2.1. Булевы схемы и последовательности операций. Начиная с этого места, мы будем, в основном, иметь дело с булевьми функциями, т.е. функциями вида $f: \mathbf{B}^{n} \rightarrow \mathbf{B}^{m}$. Выберем некоторое множество $\mathscr{A}$ таких функций и будем использовать их для построения других функций. Это множество, назьваемое базисом, должно содержать тождественную функцию $I=I_{\mathbf{B}}: \mathbf{B} \rightarrow \mathbf{B}$ (в целях технического удобства). Обычно используется следуюший стандартньй базис: $\mathscr{C}=\{I, 0, \neg, \wedge\}$ (где $\neg-$ отрицание, а $\wedge$ - функция “и”). Этот базис полон, т.е. любую булеву функцию можно представить как суперпозищию элементов базиса.

Пусть $F: \mathbf{B}^{n} \rightarrow \mathbf{B}^{m}$ - произвольная функция. Булева схема для $F$ - это процедура, которая преобразует входное слово $x \in \mathbf{B}^{n}$ в выходное слово $y=F(x) \in \mathbf{B}^{m}$, используя вспомогательные булевы переменные $z_{1}, \ldots, z_{l}$ согласно следуюшим правилам:

1) скопировать $x$ в $\left(z_{1}, \ldots, z_{n}\right)$;

2) вычислить последовательно значения переменных $z_{n+1}, \ldots, z_{l}$, применяя функции базиса $f_{1}, \ldots, f_{L} \in \mathscr{A}\left(f_{i}: \mathbf{B}^{n_{i}} \rightarrow \mathbf{B}^{m_{i}}\right)$ к уже вычисленньп (на данньй момент) значениям:

$$
\left(z_{k_{i-1}+1}, \ldots, z_{k_{i}}\right)=f_{i}\left(z_{\alpha(i, 1)}, \ldots, z_{\alpha\left(i, n_{i}\right)}\right), \quad \alpha(i, 1), \ldots, \alpha\left(i, n_{i}\right) \leqslant k_{i-1}
$$

где $k_{0}=n, k_{i}=k_{i-1}+m_{i}$;

3) прочитать $y$ из $\left(z_{\beta(1)}, \ldots, z_{\beta(m)}\right)$.

Таким образом, булева схема определяется последовательностью функций $f_{1}, \ldots, f_{L} \in \mathscr{A}$ и числами $\alpha(i, j), \beta(j)$. Число $L$ называется размером схемы. Для большей обшности можно допустить, что $F$ - частичная функиия $\mathbf{B}^{n} \rightarrow \mathbf{B}^{m}$, т.е. функция вида $N \rightarrow \mathbf{B}^{m}$, где $N \subseteq \mathbf{B}^{n}$. В этом случае выходное слово $y$ должно совпадать с $F(x)$ для всех $x \in N$.

Пример 2.1. Рассмотрим сложение двух двоичных чисел длины 1:

$$
F:\left(x_{1}, x_{2}\right) \mapsto\left(x_{1} \wedge x_{2},\left(x_{1} \vee x_{2}\right) \wedge \neg\left(x_{1} \wedge x_{2}\right)\right) .
$$

Эту функщию можно представить следующей булевой схемой в базисе $\mathscr{A}=\{I, \neg, \wedge, \vee\}$ :

$$
\left(z_{1}, z_{2}\right)=x, \quad z_{3}=z_{1} \wedge z_{2}, \quad z_{4}=z_{1} \vee z_{2}, \quad z_{5}=\neg z_{3}, \quad z_{6}=z_{4} \wedge z_{5}, \quad y=\left(z_{3}, z_{6}\right) .
$$

Данное вьше определение булевой схемы является обшепринятым. Теперь мы собираемся определить немного другую, но эквивалентную вычислительную модель, которая более удобна для дальнейших обобщений. В новой модели значения переменных $z_{i}$ меняются на каждом шаге (а не вычисляются раз и навсегда). Это больше похоже на работу реального компьютера.

Пусть $\Delta=\{1, \ldots, l\}$ - память, которая будет использоваться при вычислении. Каждый элемент памяти (бит) $i \in \Delta$ соответствует булевой переменной $z_{i}$. Любое упорядоченное множество битов называется регистром. С каждым регистром $A=\left(j_{1}, \ldots, j_{n}\right)$ связана переменная $z_{A}=\left(z_{j_{1}}, \ldots, z_{j_{n}}\right)$, принимающая значения из $\mathbf{B}^{n}$. Если дана произвольная булева функция $g: \mathbf{B}^{n} \rightarrow \mathbf{B}^{n}$, то можно определить ее действие $g[A]: z_{A} \mapsto g\left(z_{A}\right)$ на множестве состояний регистра $A$. Мы можем рассматривать $g[A]$ как отображение на полном множестве состояний памяти, $\Gamma=\mathbf{B}^{\Delta}$. Такие отображения назьваются операциями. 
Рассмотрим более общую ситуацию: пусть $\Delta, \Delta^{\prime}$ - две памяти, $A \subseteq \Delta, A^{\prime} \subseteq \Delta^{\prime}-$ два регистра, $g: \mathbf{B}^{|A|} \rightarrow \mathbf{B}^{\left|A^{\prime}\right|}$ - булева функция. Предположим, что $\Delta \backslash A=\Delta^{\prime} \backslash A^{\prime}$. Тогда $g\left[A^{\prime} ; A\right]: \mathbf{B}^{\Delta} \rightarrow \mathbf{B}^{\Delta^{\prime}}$. Таким образом, $g[A]$ - сокращение для $g[A ; A]$.

Зафиксируем в качестве базиса некоторое множество булевых функций $\mathscr{A}$. Введем обозначение $\nabla: \mathbf{B} \rightarrow \mathbf{B}^{2}: a \mapsto(a, a)$. Новая модель - это процедура следующего вида:

1) поместить входное слово в некоторьй регистр $X=\Delta_{0}$;

2) выполнить последовательно некоторые операции $g_{1}\left[A_{1}^{\prime} ; A_{1}\right], \ldots, g_{L}\left[A_{L}^{\prime} ; A_{L}\right]$ $\left(g_{i} \in \mathscr{A} \cup\{\nabla\}\right) ;$ предполагается, что $A_{i} \subseteq \Delta_{i-1}, \Delta_{i}=\left(\Delta_{i-1} \backslash A_{i}\right) \cup A_{i}^{\prime}$;

3) прочитать выходное слово из некоторого регистра $Y \subseteq \Delta_{L}$.

Пример 2.1 можно представить как следующую композицию операций (нужно читать справа налево):

$$
\wedge[6 ; 4,5] \neg[5] \quad \nabla[3,5 ; 3] \quad \vee[4 ; 1,2] \quad \wedge[3 ; 3,4] \quad \nabla[2,4 ; 2] \quad \nabla[1,3 ; 1]
$$

Ясно, что любую булеву схему размера $L$ можно преобразовать в последовательность из $c L$ операций (где $c$ - наибольшее число битов, задействованных в базисной функции). Обратно, любую последовательность из $L$ операций можно промоделировать булевой схемой размера $L$. Таким образом, обе модели эквивалентны.

Схемы и алгоритмы. Булева схема всегда работает с входными словами фиксированной длины. Однако вычислительные задачи, как правило, формулируются для слов переменной длины - рассмотрим, например, задачу о сложении двоичных чисел $\left(x, x^{\prime}\right) \mapsto x+x^{\prime}$. Любая разумная вычислительная задача $F$ может быть представлена как последовательность функций $\left.F\right|_{s}: \mathbf{B}^{s} \rightarrow \mathbf{B}^{\text {poly }(s)}$, каждая из которых соответствует определенному размеру входного слова $s$. Для каждой функции $\left.F\right|_{s}$ требуется своя булева схема. Если для задачи $F$ существует полиномиальный алгоритм, то каждая функция $\left.F\right|_{s}$ может быть вычислена схемой $\Phi_{s}$ paзмера $\operatorname{poly}(s)$. B самом деле, компьютер (точнее, машину Тьюринга), работаюший с ограниченными ресурсами $\left(\right.$ память $\times$ время $\leqslant t \times t$ ), можно промоделировать булевой схемой размера $O\left(t^{2}\right)$.

Обратное, вообще говоря, неверно. Сушествование полиномиальной схемы $\Phi_{s}$ для каждой функции $\left.F\right|_{s}$ не обязательно означает, что полная функция $F$ полиномиально вычислима (или даже просто вычислима). Для этого требуется, чтобы схему $\Phi_{s}$ можно было построить эффективно. Точнее говоря, функция $s \mapsto \Phi_{s}$ должна быть вычислима на машине Тьюринга за полиномиальное время. Семейство функций $\left(\Phi_{s}\right)$, удовлетворяюшее этому условию, назьвается однородным. Таким образом, машина Тьюринга порождает схему, а схема вьчисляет функцию.

Эта двухуровневая конструкция особенно хороша для определения нестандартных вычислительных моделей, включая квантовые вычисления. В разделе 4 мы определим квантовые схемы - некоторые теоретические устройства, вычисляющие булевы функции. Как и любой комбинаторньй объект, квантовую схему можно описать (закодировать) при помоши двоичного слова. ${ }^{4}$ Под квантовым алгоритмом для задачи $F$ мы будем понимать обычный (классический) алгоритм, которьй строит квантовую схему $\Psi_{s}$ для каждой функции $\left.F\right|_{s}$.

\footnotetext{
${ }^{4} \mathrm{~B}$ комбинаторных терминах описывается устройство, но не процесс работы квантовой схемы.
} 
2.2. Обратимые вычисления. Модели, определенные вьше, так же, как и реальные компьютеры, допускают необратимые операции. В самом деле, даже очистка (обнуление) одного бита - необратимое действие. В этом разделе мы покажем, что подобных операший можно избежать. Понятие обратимого вычисления ввел Lecerf [50], а затем, независимо, Bennett [51]. Оно является важным связующим звеном между булевьми схемами и квантовым вычислением.

Прежде всего, обратимое вычисление должно использовать только биективные отображения $g_{i}: \mathbf{B}^{n} \rightarrow \mathbf{B}^{n}$, т.е. перестановки на булевых кубах. Простой, но важньй пример - биективное отображение $\bigoplus_{n}:(u, v) \mapsto(u, v \oplus u)$ на $\mathbf{B}^{2 n}$. (Здесь “ $\oplus$ ” обозначает побитное сложение по модулю 2.) Очевидно, операция $\oplus_{n}[U, V]$ эквивалентна применению отображения $\oplus=\oplus_{1}$ к каждой паре битов. Отображение $\oplus$ позволяет скопировать один бит в другой при условии, что значение второго бита сначала равно нулю. Более обший пример: рассмотрим произвольную функцию $F: N \rightarrow \mathbf{B}^{m}$ $\left(N \subseteq \mathbf{B}^{n}\right)$. Следующее отображение является биекцией:

$$
F_{\oplus}: N \times \mathbf{B}^{m} \rightarrow N \times \mathbf{B}^{m}: \quad(u, v) \mapsto(u, v \oplus F(u))
$$

Теперь понятно, как моделировать любую булеву схему последовательностью обратимых операций. Прежде всего, нужно иметь достаточно большой запас "чистых" (имеюших нулевое значение) битов. Добавим их к входному слову и произведем следуюшую последовательность операций:

$$
\left(f_{i}\right)_{\oplus}\left[\alpha(i, 1), \ldots, \alpha\left(i, n_{i}\right), k_{i-1}+1, \ldots, k_{i}\right] \quad(i=1, \ldots, L) .
$$

Однако это вычисление не является полностью обратимым. Дело в том, что кроме выходного слова оно производит некоторый "мусор", т.е. вспомогательные биты не остаются чистыми. Без этого мусора вычисление нельзя прокрутить назад, чтобы вернуться от выходного слова к входному. ${ }^{5}$ Мы увидим, что мусор препятствует использованию классической последовательности операций в качестве подпрограммы для квантового компьютера - вот почему мы уделяем обратимости такое внимание. К счастью, возникновения мусора можно избежать.

Теперь мы можем дать точное определение обратимого вычисления, т.е. вычисления без мусора. В последуюших построениях мы предполагаем, что память $\Delta$ является объединением двух непересекающихся регистров: входного-выходного регистра $X$ и рабочего регистра $W$. Таким образом, каждое состояние памяти можно записать как $(x, w)$, где $x \in \mathbf{B}^{X}, w \in \mathbf{B}^{W}$.

ОПрЕДЕЛЕНИЕ 2.2. Пусть $G: N \rightarrow M\left(N, M \subseteq \mathbf{B}^{n}\right)$ - произвольная биекция. Говорят, что обратимая схема, т.е. последовательность биективных операций $g_{i}\left[A_{i}\right]$ $(i=1, \ldots, L)$, представляет $G$, если их композиция $g_{L}\left[A_{L}\right] \cdots g_{1}\left[A_{1}\right]$ отображает $(x, 0)$ в $(G(x), 0)$ для каждого $x \in N$.

\footnotetext{
${ }^{5}$ Реальные компьютеры тоже создают своего рода "мусор" - тепло. Необратимость элементарных вычислительных операций определяет теоретическую нижнюю границу для количества выделяемого тепла [52]. (Впрочем, существующие в настоящее время компьютеры превосходят эту границу на много порядков.)
} 
ЗАмЕчАнИЕ. Мы считаем, что любые перестановки битов являются "бесплатными", т.е. не включаются в размер схемы. Это соглашение эквивалентно тому, чтобы допустить операции вида $g\left[A^{\prime} ; A\right]$, а не только $g[A]$. С другой стороны, два бита можно поменять местами следуюшим образом:

$$
[1 \leftrightarrow 2]=\oplus[1,2] \oplus[2,1] \oplus[1,2]
$$

ПреДЛОЖЕНИЕ 2.3. Предположим, что функиия $F: N \rightarrow \mathbf{B}^{m}\left(N \subseteq \mathbf{B}^{n}\right)$ вьчислима в базисе $\mathscr{A}$ посредством булевой схемы размера L. Тогда $F_{\oplus}$ можно представить в базисе $\mathscr{A}_{\oplus}=\left\{f_{\oplus}: f \in \mathscr{A}\right\}$ обратимой схемой размера $2 L+m$.

ДокАЗАТЕльство. Как показано выше, булеву схему можно заменить на последовательность из $L$ операций в базисе $\mathscr{A}_{\oplus}$. Действие этой последовательности операций записьвается как $G[U, W]:(u, 0) \mapsto(u, w)$, где $w$ содержит $F(x)$ в определенном подрегистре $Y \subseteq W$. Чтобы построить обратимую схему для $F_{\oplus}$, нам потребуется вспомогательньй (рабочий) регистр $V$ размера $m$. Теперь любое состояние памяти записьвается в виде $(u, v, w)$, где $u, v$ и $w$-содержимое регистров $U, V$ и $W$, соответственно. Требуемое обратимое вычисление дается композищией отображений

$$
(G[U, W])^{-1} \oplus_{m}[Y, V] \quad G[U, W]: \quad(u, v, 0) \mapsto(u, v \oplus F(u), 0) \quad\left(u \in N, v \in \mathbf{B}^{m}\right) .
$$

Действительно, отображение $G[U, W]$ вьгисляет слово $F(u)$, отображение $\oplus_{m}[Y, V]$ прибавляет его к $v$ (по модулю 2$)$, а $(G[U, W])^{-1}$ убирает мусор, т.е. делает $w$ равньм нулю. (Заметим, что $\oplus=I_{\oplus} \in \mathscr{A}_{\oplus}$, поскольку $I \in \mathscr{A}$.)

ПреДЛОЖЕНИЕ 2.4. Пусть $G: N \rightarrow M\left(N, M \subseteq \mathbf{B}^{n}\right)$ - биекция. Предположим, что $G$ и $G^{-1}$ вычислимы в базисе $\mathscr{A}$ булевьми схемами размера $L$ и $L^{\prime}$, соответственно. Тогда $G$ можно представить обратимой схемой размера $2 L+2 L^{\prime}+2 n$ в базисе $\mathscr{A}_{\oplus}$.

ДОКАЗАТЕЛЬСТВО. Пусть $X$ - входной-выходной регистр, а $Y$ - вспомогательный регистр того же размера $n$. Необходимо добавить еше один рабочий регистр $W$, которьй будет использоваться в обратимых схемах для $G_{\oplus}$ и $\left(G^{-1}\right)_{\oplus}$. Согласно предложению 2.3 , эти схемы имеют размер $2 L+n$ и $2 L^{\prime}+n$, соответственно. Обратимая схема для $G$ имеет вид

$$
[X \leftrightarrow Y] \quad\left(G^{-1}\right)_{\oplus}[Y, X] \quad G_{\oplus}[X, Y]
$$

В самом деле, $(x, 0) \mapsto(x, G(x)) \mapsto(0, G(x)) \mapsto(G(x), 0)$.

СлЕДСТВИЕ. Базис $\mathscr{C}_{\oplus}$ полон для обратимьх вычислений.

Элемент $\neg \oplus \in \mathscr{C}_{\oplus}$ можно заменить на $\neg$. Таким образом, мы получаем другой полный базис

$$
\mathscr{R}=\left\{I, \neg, \oplus, \wedge_{\oplus}\right\}
$$

(Отображение $\wedge_{\oplus}$ называется элементом Тоффоли (Toffoli gate).) Мы всегда будем использовать именно этот базис, если только речь не зайдет о так назьваемых относительных вычислениях, т.е. вычислениях с оракулом. 
Пусть $F: \mathbf{B}^{n} \rightarrow \mathbf{B}^{m}$ - произвольная функщия, возможно, частичная. Обратимой схемой с оракулом $F$ мы будем назьвать любую обратимую схему в базисе $\mathscr{R} \cup\left\{F_{\oplus}\right\}$. Это определение является естественным ввиду предложения 2.3.

В дальнейшем мы будем рассматривать лиш один частньй случай оракула. Пусть $F: \mathbf{Z}^{k} \times M \rightarrow \mathbf{B}^{n}$ - функция из определения задачи о стабилизаторе (см. раздел 1$)$. Положим

$$
\mathscr{Z}_{s}^{k}=\left\{g \in \mathbf{Z}^{k}: \operatorname{size}(g), \operatorname{size}(-g) \leqslant s\right\} .
$$

Множество $\mathscr{Z}_{s}^{k}$ можно отождествить с определенным подмножеством $\mathbf{B}^{s}$. Обозначим через $\left.F\right|_{s+n}$ ограничение $F$ на $\mathscr{Z}_{s}^{k} \times M$. Под вычислением с оракулом $F$ мы будем понимать вычисление в базисе $\mathscr{R} \cup\left\{\left(\left.F\right|_{s+n}\right)_{\oplus}\right\}$, где $s=\operatorname{poly}(k+n)$. Для нашего квантового алгоритма (см. раздел 6 ) будет нужна не сама функция $F$, а следующая биекция:

$$
G: \mathbf{Z}^{k} \times M \rightarrow \mathbf{Z}^{k} \times M:(g, x) \mapsto(g, F(g, x))
$$

Заметим, что $G^{-1}:(g, x) \mapsto(g, F(-g, x))$. Функцию $\left.G\right|_{s+n}$ (ограничение $G$ ) можно рассматривать как частичную перестановку на множестве $\mathbf{B}^{s} \times \mathbf{B}^{n}$. Предложение 2.4 позволяет выразить $\left.G\right|_{s+n}$ через $\left.F\right|_{s+n}$.

Лемма 2.5. Функиия $\left.G\right|_{s+n}$ может бъть представлена обратимой схемой размера $O(n)$ с оракулом $\left.F\right|_{s+n}$. Элемент $\left(\left.F\right|_{s+n}\right)_{\oplus}$ входит в эту схему дваж$\partial b l$.

\section{3. Квантовый формализм}

3.1. Основные понятия и обозначения. Классическая система описывается конечным множеством состояний $\Gamma$. (В теоретико-вычислительном контексте, $\Gamma=\mathbf{B}^{\Delta}$ - множество состояний памяти компьютера $\Delta$.) Соответствующая квантовая система описьвается унитарньм пространством $\bar{\Gamma}=\mathbf{C}^{\Gamma}$ со стандартным ортонормированным базисом $\{|a\rangle: a \in \Gamma\}$. Чистое квантовое состояние - это единичный вектор $|\psi\rangle \in \bar{\Gamma}$, заданньй с точностью до фазового множителя $c:|c|=1$. (Другими словами, квантовое состояние - это одномерное подпространство в $\bar{\Gamma}$.) Эволюция квантового состояния (за определенное время) дается формулой $|\psi\rangle \mapsto U|\psi\rangle$, где $U$ - унитарньй оператор. Каждой биекции $G: \Gamma \rightarrow \Gamma$ соответствует унитарньй оператор $\widehat{G}$, действующий по правилу $\widehat{G}|a\rangle=|G(a)\rangle$. Такие операторы называются классическими.

Пример 3.1. Система с двумерным пространством состояний $\mathscr{B}=\overline{\mathbf{B}}$ назьвается кубитом (квантовым битом). Матрищы Паули

$$
\sigma_{x}=\left(\begin{array}{cc}
0 & 1 \\
1 & 0
\end{array}\right), \quad \sigma_{y}=\left(\begin{array}{cc}
0 & -\mathrm{i} \\
\mathrm{i} & 0
\end{array}\right), \quad \sigma_{z}=\left(\begin{array}{cc}
1 & 0 \\
0 & -1
\end{array}\right)
$$

яВляются унитарными; при этом оператор $\sigma_{x}=\hat{ᄀ}-$ классический, а $\sigma_{y}$ и $\sigma_{z}-$ нет.

Векторы пространства $\mathscr{N}=\bar{\Gamma}$ обычно обозначаются как $|\xi\rangle$, даже если символ, стояший в скобках (в данном случае, $\xi$ ), никогда не используется отдельно. Скалярное произведение двух векторов $|\xi\rangle,|\eta\rangle \in \mathscr{N}$ обозначается через $\langle\xi \mid \eta\rangle$. Таким образом, $\langle\xi|$ 
обозначает линейньй функционал $|\eta\rangle \mapsto\langle\xi \mid \eta\rangle$ на $\mathscr{N}$. Пространство таких функционалов обозначается через $\mathscr{N}^{*}$. В координатных обозначениях, $|\xi\rangle=\sum_{j \in \Gamma} \xi_{(j)}|j\rangle$, $\langle\xi|=\sum_{j \in \Gamma} \xi_{(j)}^{\star}\langle j|$, или

$$
|\xi\rangle=\left(\begin{array}{c}
\xi_{(1)} \\
\vdots \\
\xi_{(k)}
\end{array}\right), \quad\langle\xi|=\left(\xi_{(1)}^{\star}, \ldots, \xi_{(\kappa)}^{\star}\right) .
$$

(Здесь звездочка * обозначает комплексное сопряжение.) Если $|\xi\rangle,|\eta\rangle \in \mathscr{N}$, то $|\xi\rangle\langle\eta|$ является элементом $\mathscr{N} \otimes \mathscr{N}^{*}$ и, таким образом, может рассматриваться как линейньй оператор на $\mathscr{N}$. Результат применения линейного оператора $A: \mathscr{N} \rightarrow \mathscr{N}$ к вектору $|\xi\rangle$ обозначается через $A|\xi\rangle=|A \xi\rangle$. Таким образом,

$$
\langle\xi \mid A \eta\rangle=\langle\xi|A| \eta\rangle=\left\langle A^{\dagger} \xi \mid \eta\right\rangle, \quad\langle\xi| A=\left\langle A^{\dagger} \xi\right|,
$$

где $A^{\dagger}$ - оператор, эрмитово сопряженный к $A$.

Множество линейных операторов на $\mathscr{N}$ обозначается через $\mathbf{L}(\mathscr{N})$. Это конечномерная $C^{\star}$-алгебра, называемая алгеброй наблюдаемых. Група унитарных операторов обозначается через $\mathrm{U}(\mathscr{N})$. Пространство линейных операторов $\mathscr{N} \rightarrow \mathscr{N}^{\prime}$ обозначается через $\mathbf{L}\left(\mathscr{N}, \mathscr{N}^{\prime}\right)$.

Пусть П $\mathscr{M}|\xi\rangle$ обозначает ортогональную проекцию вектора $|\xi\rangle$ на линейное подпространство $\mathscr{M} \subseteq \mathscr{N}$. Оператор проектирования П $\mathscr{M}$ можно представить как $\sum_{j=1}^{k}\left|e_{j}\right\rangle\left\langle e_{j}\right|$, где $\left(\left|e_{j}\right\rangle: j=1, \ldots, k\right)$ - произвольный ортонормированный базис в $\mathscr{M}$.

Для формального понимания квантовой механики нужны две веши: вероятностная интерпретация и связь между системой и ее подсистемами. С математической точки зрения, вероятностная интерпретация - это просто определение некоторой функции под названием "вероятность". После того, как такое определение сформулировано, можно убедиться, что эта функция действительно обладает обычными свойствами вероятности. Пока мы просто дадим определение. Аналогия с классическим случаем полностью выявится в разделе 5 , где будут введены условные вероятности.

Классическая вероятность $\mathrm{P}(\mu, M)=\mu(M)=\sum_{j \in M} \mu(j)$ есть функция двух аргументов: вероятностной меры $\mu$ на Г и подмножества $M \subseteq \Gamma{ }^{6}$ Соответственно, квантовая вероятность зависит от квантового состояния $|\xi\rangle$ и линейного подпространства $\mathscr{M} \subseteq \mathscr{N}=\bar{\Gamma}$.

$$
\mathrm{P}(\xi, \mathscr{M})=\langle\xi|\Pi \mathscr{M}| \xi\rangle
$$

Эту величину можно также представить как $\operatorname{Tr}\left(\rho \Pi_{\mathscr{M}}\right)$, где $\rho=|\xi\rangle\langle\xi|$ - оператор плотности, отвечаюший состоянию $|\xi\rangle$. Общее определение: оператор плотности, или смешанное состояние, есть положительньй эрмитов оператор $\rho \in \mathbf{L}(\mathscr{N})$ со следом 1 ; множество таких операторов обозначается через $\mathbf{D}(\mathscr{N})$. В этом случае

$$
\mathrm{P}(\rho, \mathscr{M})=\operatorname{Tr}(\rho \Pi \mathscr{M}) .
$$

\footnotetext{
${ }^{6}$ Поскольку множество Г конечно, вероятностная мера есть просто положительная функция $\mu: \Gamma \rightarrow \mathbf{R}$ такая, что $\sum_{j \in \Gamma} \mu(j)=1$.
} 
Операторы плотности естественно рассматривать как линейные функционалы на алгебре $\mathbf{L}(\mathscr{N})$. Пространство таких функционалов $\mathbf{L}(\mathscr{N})^{*}$ отождествляется с $\mathbf{L}(\mathscr{N})$ посредством комплексного скалярного произведения $(A, B)=\operatorname{Tr}(A B)$.

Формула (9) включает в себя классическую вероятность. В самом деле, пусть $\mathscr{M}$ - пространство, порожденное стандартньми векторами $|a\rangle: a \in M$. Пусть также $\rho=\sum_{a \in \Gamma} \mu(a)|a\rangle\langle a|$, где $\mu$-вероятностная мера на $\Gamma$. Тогда $\mathrm{P}(\rho, \mathscr{M})=\mathrm{P}(\mu, M)$. Как и классическая вероятность, квантовая вероятность аддитивна. А именно, если $\mathscr{M}_{1}, \mathscr{M}_{2}$ - ортогональные подпространства, то

$$
\mathrm{P}\left(\rho, \mathscr{M}_{1} \oplus \mathscr{M}_{2}\right)=\mathrm{P}\left(\rho, \mathscr{M}_{1}\right)+\mathrm{P}\left(\rho, \mathscr{M}_{2}\right) .
$$

Типичная задача квантовой механики выплядит следующим образом. Дано некоторое начальное состояние $\rho_{0} \in \mathbf{D}(\mathscr{N})$. K нему применяется унитарный оператор $U \in \mathbf{U}(\mathscr{N})$, действуюший по правилу $\rho_{0} \mapsto \rho=U \rho_{0} U^{\dagger}$. (Это соответствует формуле $|\psi\rangle \mapsto U|\psi\rangle$ для чистых состояний.) Требуется найти числа $\mathrm{P}\left(\rho, \mathscr{V}_{j}\right)$, где $\left\{\mathscr{V}_{j}\right\}$ - некоторое множество взаимно ортогональных подпространств в $\mathscr{N}$ (индекс $j$ пробегает конечное множество $\Omega$ ). Эти числа можно интерпретировать как вероятности различных исходов определенного физического процесса. В такой ситуации говорят, что определена квантовая переменная $z_{\Omega}$, принимаюшая значения $j \in \Omega$ с вероятностями $\mathrm{P}\left(\rho, \mathscr{V}_{j}\right)$. Очевидно, что $\sum_{j \in \Omega} \mathrm{P}\left(\rho, \mathscr{V}_{j}\right)+\mathrm{P}(\rho, \mathscr{V} /)=1$, где $\mathscr{V} ?$ дополнение к $\bigoplus_{j \in \Omega} \mathscr{V}_{j}$. Если $\mathscr{A}$ - предикат на $\Omega$ (т.е. функция $\Omega \rightarrow\{$ истина, ложь $\}$ ), то $\operatorname{Prob}_{\rho}\left[\mathscr{A}\left(z_{\Omega}\right)\right]$ обозначает вероятность того, что $\mathscr{A}\left(z_{\Omega}\right)$ истинно,

$$
\operatorname{Prob}_{\rho}\left[\mathscr{A}\left(z_{\Omega}\right)\right]=\sum_{j \in \Omega: \mathscr{A}(j)} \mathrm{P}\left(\rho, \mathscr{V}_{j}\right) .
$$

Например, $\operatorname{Prob}_{\rho}\left[z_{\Omega}=k\right]=\mathrm{P}\left(\rho, \mathscr{V}_{k}\right)$. Обозначение $\operatorname{Prob}_{\rho}[\ldots]$ удобно тем, что оно выражает интуитивньй смысл вероятности.

Начиная с раздела 7 , мы будем рассматривать комбинированные классически-квантовые системы. Комбинированное пространство - это любой конечньй набор унитарных пространств $\mathscr{N}=\left(\mathscr{N}_{j}: j \in \Omega\right)$. Ему соответствуют суммарное пространство $\overline{\mathscr{N}}=\bigoplus_{j \in \Omega} \mathscr{N}_{j}$ и алгебра наблюдаемых $\mathbf{L}(\mathscr{N})=\bigoplus_{j \in \Omega} \mathbf{L}\left(N_{j}\right) \subseteq \mathbf{L}(\overline{\mathscr{N}})$. Известно, что любая конечномерная $C^{\star}$-алгебра однозначно разлагается в прямую сумму матричных алгебр. Таким образом, комбинированные пространства находятся во взаимно-однозначном соответствии с конечномерными $C^{\star}$-алгебрами. Полезно также иметь ввиду следуюший факт. Пусть $\mathscr{L}$ - унитарное пространство, $\mathscr{H} \subseteq \mathbf{L}(\mathscr{L})$ - подалгебра с инволюцией, содержашая единищу. Тогда

$$
\mathscr{L}=\bigoplus_{j} \mathscr{M}_{j} \otimes \mathscr{F}_{j}, \quad \mathscr{H}=\bigoplus_{j} \mathbf{L}\left(\mathscr{M}_{j}\right) \otimes 1_{\mathscr{F}_{j}} .
$$

Чистое состояние в комбинированном пространстве $\mathscr{N}=\left(\mathscr{N}_{j}: j \in \Omega\right)$ есть произвольньй единичньй вектор $|\xi\rangle \in \mathscr{N}_{j}$ (с точностью до фазового множителя). Смешанное состояние - это элемент множества $\mathbf{D}(\mathscr{N})=\mathbf{D}(\overline{\mathscr{N}}) \cap \mathbf{L}(\mathscr{N})$. Под комбинированным подпространством $\mathscr{M} \subseteq \mathscr{N}$ понимается набор линейных подпространств $\left(\mathscr{M}_{j} \subseteq \mathscr{N}_{j}: j \in \Omega\right)$. Соответствуюшая вероятность дается формулой (9). 
Любой физический процесс в комбинированной системе можно рассматривать в терминах унитарного пространства $\overline{\mathscr{N}}$. В этом смысле понятие комбинированного пространства не является обязательным. Однако знание “классических степеней свободы" в задаче бывает полезным.

Теперь рассмотрим объединение двух квантовых подсистем с пространствами состояний $\mathscr{N}_{1}=\bar{\Gamma}_{1}$ и $\mathscr{N}_{2}=\bar{\Gamma}_{2}$. Множество классических состояний всей системы есть $\Gamma=\Gamma_{1} \times \Gamma_{2}$, тогда как соответствуюшее унитарное пространство имеет вид $\mathscr{N}=\mathscr{N}_{1} \otimes \mathscr{N}_{2}$. Два вектора, $\left|\xi_{1}\right\rangle \in \mathscr{N}_{1}$ и $\left|\xi_{2}\right\rangle \in \mathscr{N}_{2}$, можно объединить в вектор $\left|\xi_{1}, \xi_{2}\right\rangle=\left|\xi_{1}\right\rangle \otimes\left|\xi_{2}\right\rangle \in \mathscr{N}$. Можно также определить тензорное произведение линейных подпространств, линейных операторов, унитарных операторов и смешанных состояний. Ясно, что

$$
\mathrm{P}\left(\rho_{1} \otimes \rho_{2}, \mathscr{M}_{1} \otimes \mathscr{M}_{2}\right)=\mathrm{P}\left(\rho_{1}, \mathscr{M}_{1}\right) \mathrm{P}\left(\rho_{2}, \mathscr{M}_{2}\right)
$$

(В классическом случае, $\rho_{1} \otimes \rho_{2}$ соответствует совместному распределению двух независимых случайных величин.)

Принципиальная разница между классической и квантовой механикой заключается в том, что квантовое состояние всей системы невозможно, вообще говоря, разложить на состояния ее подсистем. Не существует даже никакого естественного отображения $\mathscr{N} \rightarrow \mathscr{N}_{1}$. Имеется, однако, другое естественное отображение - частичный след

$$
\operatorname{Tr}_{\mathscr{N}_{2}}: \mathbf{L}\left(\mathscr{N}_{1} \otimes \mathscr{N}_{2}\right) \rightarrow \mathbf{L}\left(\mathscr{N}_{1}\right), \quad \operatorname{Tr}_{\mathscr{N}_{2}} X=\sum_{a, b \in \Gamma_{1}}\left(\sum_{c \in \Gamma_{2}}\langle a, c|X| b, c\rangle\right)|a\rangle\langle b|
$$

Переход от смешанного состояния $\rho \in \mathbf{D}(\mathscr{N})$ к смешанному состоянию $\rho_{1}=\operatorname{Tr}_{\mathscr{N}_{2}} \rho \in$ $\mathbf{D}\left(\mathscr{N}_{1}\right)$ интерпретируется как исключение из рассмотрения второй подсистемы. (Эта операция аналогична проектированию совместного распределения на одну из переменных.) Переход от $\rho$ к $\rho_{1}$ имеет смысл, если мы интересуемся вероятностями вида $\mathrm{P}\left(U \rho U^{\dagger}, \mathscr{M}\right)$, где $U=U_{1} \otimes U_{2}$ (т.е. подсистемы эволюционируют независимо), и $\mathscr{M}=\mathscr{M}_{1} \otimes \mathscr{N}_{2}$ (т.е. мы наблюдаем только первую подсистему). В самом деле,

$$
\mathrm{P}\left(\rho, \mathscr{M}_{1} \otimes \mathscr{N}_{2}\right)=\mathrm{P}\left(\operatorname{Tr}_{\mathscr{N}_{2}} \rho, \mathscr{M}_{1}\right), \quad \operatorname{Tr}_{\mathscr{N}_{2}}\left(\left(U_{1} \otimes U_{2}\right) \rho\left(U_{1} \otimes U_{2}\right)^{\dagger}\right)=U_{1}\left(\operatorname{Tr}_{\mathscr{N}_{2}} \rho\right) U_{1}^{\dagger}
$$

Стоит упомянуть три свойства частичного следа. Во-первых, любое смешанное состояние $\rho \in \mathbf{D}(\mathscr{N})$ можно представить в виде

$$
\rho=\operatorname{Tr}_{\mathscr{N}}(|\xi\rangle\langle\xi|), \quad|\xi\rangle=\sqrt{\rho} \in \mathbf{L}(\mathscr{N})=\mathscr{N} \otimes \mathscr{N}^{*}
$$

Во-вторых, состояние $\rho_{1}=\operatorname{Tr}_{\mathscr{N}_{2}} \rho\left(\rho \in \mathbf{D}\left(\mathscr{N}_{1} \otimes \mathscr{N}_{2}\right)\right)$ является чистым тогда и только тогда, когда $\rho=\left(\left|\xi_{1}\right\rangle\left\langle\xi_{1}\right|\right) \otimes \rho_{2}$, где $\left|\xi_{1}\right\rangle \in \mathscr{N}_{1}, \rho_{2} \in \mathbf{D}\left(\mathscr{N}_{2}\right)$. В-третьих, имеет место следующее свойство.

ЛЕмма 3.2. Пусть $|\xi\rangle \in \mathscr{N}_{1} \otimes \mathscr{N}_{2}, \rho_{1}=\operatorname{Tr}_{\mathscr{N}_{2}}(|\xi\rangle\langle\xi|), \rho_{2}=\operatorname{Tr}_{\mathscr{N}_{1}}(|\xi\rangle\langle\xi|)$. Тогда ненулевые собственные значения $\rho_{1}$ и $\rho_{2}$ совпадают.

ДоказАтельство. Диагонализуем $\rho_{1}$ и напишем $|\xi\rangle=\sum_{j}\left|j, \eta_{j}\right\rangle$, где $\left|\eta_{j}\right\rangle \in \mathscr{N}_{2}$. Тогда $\left\langle\eta_{j} \mid \eta_{k}\right\rangle=\lambda_{j} \delta_{j k}$, где $\lambda_{j}-j$-е собственное значение $\rho_{1}$. 
3.2. Преобразования смешанных состояний. Результаты этого раздела существенно используются лишь во второй части статьи. Однако примеры допустимых преобразований (см. ниже) довольно поучительны; имеет смысл их посмотреть сразу.

Будем называть преобразованиями любые операторы вида $\mathbf{L}(\mathscr{N}) \rightarrow \mathbf{L}(\mathscr{M})$, где $\mathscr{N}$ и $\mathscr{M}$ - унитарные пространства. Пространство преобразований обозначается через $\mathbf{T}(\mathscr{N}, \mathscr{M}) ;$ используется также обозначение $\mathbf{T}(\mathscr{N})=\mathbf{T}(\mathscr{N}, \mathscr{N})$. Тождественное преобразование обозначается символом $I_{\mathscr{N}}$.

Для любого преобразования $T \in \mathbf{T}(\mathscr{N}, \mathscr{M})$ определено сопряженное (в смысле скалярного произведения $(A, B)=\operatorname{Tr}(A B))$ преобразование $T^{*} \in \mathbf{T}(\mathscr{M}, \mathscr{N})$. Каждой паре операторов $A \in \mathbf{L}(\mathscr{N}, \mathscr{M}), B \in \mathbf{L}(\mathscr{M}, \mathscr{N})$ соответствует преобразование $A \cdot B \in \mathbf{T}(\mathscr{N}, \mathscr{M})$, действующее по правилу $X \mapsto A X B$.

Как мы уже видели, преобразования вида $U \cdot U^{\dagger}: U \in \mathbf{U}(\mathscr{N})$ описьвают эволюцию смешанных состояний. Такие преобразования, называемые унитарныцми, образуют групу $\mathbf{U T}(\mathscr{N}) \cong \mathbf{U}(\mathscr{N}) / \mathbf{U}(1)$, где $\mathbf{U}(1)=\{c \in \mathbf{C}:|c|=1\}$. Преобразование унитарно тогда и только тогда, когда оно является автоморфизмом алгебры с инволющией $\mathbf{L}(\mathscr{N})$.

Кроме унитарных, существуют и другие физически реализуемые преобразования. Типичньй процесс, который приходится рассматривать, - это взаимодействие квантовой системы с окружаюшей средой. Сначала состояние системы $\rho \in \mathbf{D}(\mathscr{N})$ объединяется (т.е. умножается тензорно) с состоянием окружения $\lambda \in \mathscr{G}$. Затем происходит некоторая эволюция совместного состояния, описьваемая унитарным оператором $U: \mathscr{N} \otimes \mathscr{G} \rightarrow \mathscr{M} \otimes \mathscr{F}$. (Здесь $\mathscr{M}$ и $\mathscr{F}$ - пространства состояний системы и окружения после взаимодействия.) Наконец, окружаюшая среда исключается из рассмотрения, что дает состояние

$$
\rho^{\prime}=\operatorname{Tr}_{\mathscr{F}}\left(U(\rho \otimes \lambda) U^{\dagger}\right)=T \rho \in \mathbf{D}(\mathscr{M}) .
$$

Преобразования $T$, получаюшиеся по этой схеме, назьваются допустимылми. Заметим, что состояние $\lambda$ всегда можно считать чистым. (Достаточно заменить $\mathscr{G}$ на $\mathscr{G} \otimes \mathscr{G}^{*}$ и воспользоваться формулой (13).)

Пример 3.3. Пусть $\mathscr{N}=\mathscr{M}=\mathscr{G}=\mathscr{F}=\overline{\mathbf{B}}$. Положим $\lambda=|0\rangle\langle 0|, U=\widehat{\oplus}:| a, b\rangle \mapsto$ $|a, a \oplus b\rangle$ (здесь “Ф” обозначает сложение по модулю 2). Тогда $\left\langle a\left|\rho^{\prime}\right| b\right\rangle=\delta_{a b}\langle a|\rho| a\rangle$, т.е. преобразование $T$ выделяет из матрицы плотности $\rho$ диагональную часть. С другой стороны, $T=\frac{1}{2} I_{\mathscr{B}}+\frac{1}{2} \sigma_{z} \cdot \sigma_{z}$. Таким образом, $T$ можно интерпретировать как случайный сбой фазы: с вероятностью $\frac{1}{2}$ относительная фаза между состояниями $|0\rangle_{\text {и }}$ $|1\rangle$ меняется на $\pi$ (так действует оператор $\sigma_{z}$ ). Это есть проявление общего принципа: взаимодействие с окружающей средой играет для квантовых систем роль случайности.

Класс допустимых преобразований характеризуется очен простым набором свойств. Прежде чем их сформулировать, заметим, что

(14) $\mathbf{T}(\mathscr{N}, \mathscr{M})=\mathbf{L}(\mathscr{M}) \otimes \mathbf{L}(\mathscr{N})^{*} \cong \mathscr{M} \otimes \mathscr{N}^{*} \otimes \mathscr{M}^{*} \otimes \mathscr{N}=\mathbf{L}(\mathscr{N}, \mathscr{M}) \otimes \mathbf{L}(\mathscr{N}, \mathscr{M})^{*}$.

По аналогии с обозначением $A \cdot B$ можно написать $\mathbf{T}(\mathscr{N}, \mathscr{M})=\mathbf{L}(\mathscr{N}, \mathscr{M}) \cdot \mathbf{L}(\mathscr{N}, \mathscr{M})^{*}$. Таким образом, каждое преобразование $T \in \mathbf{T}(\mathscr{N}, \mathscr{M})$ можно рассматривать как 
оператор $\breve{T}: \mathbf{L}(\mathscr{N}, \mathscr{M}) \rightarrow \mathbf{L}(\mathscr{N}, \mathscr{M})$. В тензорных обозначениях (где по повторяюшимся индексам берется сумма)

$$
(T X)_{(i k)}=T_{(i j k l)} X_{(j l)}, \quad(\breve{T} X)_{(i j)}=T_{(i j k l)} X_{(k l)}, \quad\left(T^{*} X\right)_{(j l)}=T_{(i j k l)} X_{(i k)} .
$$

ТеОРема 3.4. Преобразование $T \in \mathbf{T}(\mathscr{N}, \mathscr{M})$ является допустимьм тогда и только тогда, когда выполнены три условия:

1) $\operatorname{Tr}(T X)=\operatorname{Tr} X$ для любого $X \in E(\mathscr{N})$, или (что равносильно) $T^{*} 1_{\mathscr{M}}=1_{\mathscr{N}}$;

2) $(T X)^{\dagger}=T X^{\dagger}$ для любого $X \in E(\mathscr{N})$, m.е. $T_{(i j k l)}^{\star}=T_{(k l i j)}$; другими словами, оператор $\breve{T}$ эрмитов;

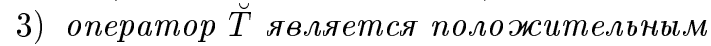

Преобразования, удовлетворяюшие условиям 2) и 3), назьваются вполне положительными.

ДОКАЗАТЕЛЬСТВо ТЕОРемЫ 3.4. Допустимость $\Rightarrow 1)-3)$. Не совсем очевидно лишь условие 3). Пусть в описанной выше схеме $\lambda=|\xi\rangle\langle\xi|$. Тогда $T_{(i j k l)}=$ $V_{(i m, j)} V_{(k m, l)}^{\star}$, где $V_{(i m, j)}=U_{(i m, j r)} \xi_{(r)}$. Следовательно, оператор $\breve{T}$ положителен.

1)-3) $\Rightarrow$ допустимость. Пусть $T \in \mathbf{T}(\mathscr{N}, \mathscr{M})$ удовлетворяет условиям 1$)-3)$. Определим каноническое представление преобразования $T$ в виде $\operatorname{Tr}_{\mathscr{F}}\left(V \cdot V^{\dagger}\right)$, где $\mathscr{F}=$ $\mathscr{M}^{*} \otimes \mathscr{N}, V \in \mathbf{L}(\mathscr{N}, \mathscr{M} \otimes \mathscr{F})$. А именно, положим $V_{(i m s, j)}=R_{(i j m s)}$, где $\breve{R}=\breve{T}^{1 / 2}$. Таким образом, $V_{(i m s, j)} V_{(k m s, l)}^{\star}=T_{(i j k l)}$. Из условия 1) вытекает, что $V$-унитарное вложение.

Пусть $\mathscr{G}=\mathscr{M} \otimes \mathscr{M}^{*}$, а $|\xi\rangle \in \mathscr{G}$ - произвольньй единичньй вектор. Рассмотрим оператор $W:|\psi, \xi\rangle \mapsto V|\psi\rangle$ с областью определения $\mathscr{N} \otimes(|\xi\rangle) \subseteq \mathscr{N} \otimes \mathscr{G}$. Пространства $\mathscr{N} \otimes \mathscr{G}$ и $\mathscr{M} \otimes \mathscr{F}$ имеют одинаковую размерность, поэтому $W$ можно продолжить до унитарного оператора $U: \mathscr{N} \otimes \mathscr{G} \rightarrow \mathscr{M} \otimes \mathscr{F}$. Осталось положить $\lambda=|\xi\rangle\langle\xi|$.

Лемма 3.5. Пусть $T \in \mathbf{T}(\mathscr{N}, \mathscr{M})$ - вполне положительное преобразование, $a V \in \mathbf{L}(\mathscr{N}, \mathscr{M} \otimes \mathscr{F}) u W \in \mathbf{L}(\mathscr{N}, \mathscr{M} \otimes \mathscr{G})$ - его представления, т.е. $T=$ $\operatorname{Tr}_{\mathscr{F}}\left(V \cdot V^{\dagger}\right)=\operatorname{Tr} \mathscr{G}\left(W \cdot W^{\dagger}\right)$. Допустим, что $\operatorname{dim} \mathscr{F} \leqslant \operatorname{dim} \mathscr{G}$. Тогда существует унитарное вложсение $U: \mathscr{F} \rightarrow \mathscr{G}$ такое, что $W=(1 \mathscr{M} \otimes U) V$.

ДокАЗАТЕЛЬство. Операторы $V$ и $W$ можно рассматривать как векторы в пространствах $\mathbf{L}(\mathscr{N}, \mathscr{M}) \otimes \mathscr{F}$ и $\mathbf{L}(\mathscr{N}, \mathscr{M}) \otimes \mathscr{G}$. (Будем использовать обозначения $|V\rangle$ и $|W\rangle$, чтобы подчеркнуть это.) Очевидно, что

$$
\operatorname{Tr}_{\mathscr{F}}(|V\rangle\langle V|)=\operatorname{Tr}_{\mathscr{G}}(|W\rangle\langle W|)=\breve{T} .
$$

Согласно лемме 3.2, ненулевые собственные значения операторов

$$
A=\operatorname{Tr}_{\mathbf{L}(\mathscr{N}, \mathscr{M})}(|V\rangle\langle V|) \text { и } B=\operatorname{Tr}_{\mathbf{L}(\mathscr{N}, \mathscr{M})}(|W\rangle\langle W|)
$$

совпадают. Следовательно, существует унитарное вложение $U: \mathscr{F} \rightarrow \mathscr{G}$, переводящее $A$ в $B$. Оператор $1_{\mathbf{L}(\mathscr{N}, \mathscr{M})} \otimes U$ переводит $|V\rangle$ в $|W\rangle$.

Следуюшее предложение, используемое в теории квантовых кодов, можно интерпретировать как невозможность невозмущающего наблюдения. (Считайте, что $\mathscr{M}-$ объект наблюдения, а $\mathscr{F}$ - пространство состояний наблюдателя.) 
ПРЕДЛОЖЕНИЕ 3.6. Пусть $Т \in \mathbf{T}(\mathscr{M}, \mathscr{M} \otimes \mathscr{F})$ - допустимое преобразование такое, что $\operatorname{Tr}_{\mathscr{F}} T=I_{\mathscr{M}}$. Тогда существует $\lambda \in \mathbf{D}(\mathscr{F})$ такое, ито $T X=X \otimes \lambda$ $($ дя любого $X \in \mathbf{L}(\mathscr{M}))$.

ДокАЗАТЕЛЬСТВо. Без уменьшения обшности можно считать, что $T=V \cdot V^{\dagger}$, где $V: \mathscr{M} \rightarrow \mathscr{M} \otimes \mathscr{F}$ - унитарное вложение. (Достаточно заменить $T$ на его каноническое представление.) Тогда для любого $|\psi\rangle \in \mathscr{M}$ состояние $\operatorname{Tr}_{\mathscr{F}}(|V \psi\rangle\langle V \psi|)=$ $\operatorname{Tr}_{\mathscr{F}} T(|\psi\rangle\langle\psi|)=|\psi\rangle\langle\psi|$ чистое, следовательно, $|V \psi\rangle=|\psi, \xi(\psi)\rangle$. Из линейности вытекает, что $|\xi(\psi)\rangle=|\xi\rangle$ не зависит от $|\psi\rangle$. Следовательно, $T X=X \otimes(|\xi\rangle\langle\xi|)$.

В заключение рассмотрим преобразования комбинированных состояний. Пусть $\mathscr{N}=\left(\mathscr{N}_{j}: j \in \Omega\right)$ и $\mathscr{M}=\left(\mathscr{M}_{k}: k \in \Theta\right)$ - комбинированные пространства. Тогда $\mathbf{T}(\mathscr{N}, \mathscr{M})=\bigoplus_{j, k} \mathbf{T}\left(\mathscr{N}_{j}, \mathscr{M}_{k}\right)$. Естественное вложение $\mathbf{T}(\mathscr{N}, \mathscr{M}) \rightarrow \mathbf{T}(\overline{\mathscr{N}}, \overline{\mathscr{M}})$ определяется формулой $P \mapsto Q_{\mathscr{M}} P Q_{\mathscr{N}}^{*}$. Здесь $Q_{\mathscr{M}}: \mathbf{L}(\mathscr{M}) \rightarrow \mathbf{L}(\overline{\mathscr{M}})$ - естественное вложение алгебр с инволющией. (Например, преобразование $T \in \mathbf{T}(\mathscr{B})$ из примера 3.3 есть образ тождественного классического преобразования $I_{\mathbf{B}}$, т.е. $T=Q_{\mathbf{B}} I_{\mathbf{B}} Q_{\mathbf{B}}^{*}$.) Комбинированное преобразование $P \in \mathbf{T}(\mathscr{N}, \mathscr{M})$ назьвается допустимьг, если преобразование $Q_{\mathscr{M}} P Q_{\mathscr{N}}^{*}-$ допустимое.

Допустимые классические преобразования имеют вид

$$
T_{(i j k l)}=t_{i j} \delta_{i k} \delta_{j l}, \quad t_{i j} \geqslant 0, \quad \sum_{i} t_{i j}=1
$$

(суммирование по повторяюшимся индексам не производится).

Пример 3.7. Пусть $\mathscr{N}=\bigoplus_{j \in \Omega} \mathscr{V}_{j}$-унитарное пространство, представленное как сумма взаимно ортогональных подпространств. Положим

$$
\operatorname{MEAS}_{\Omega}: \mathbf{L}(\mathscr{N}) \rightarrow \mathbf{L}(\mathscr{N}) \times \Omega: \quad \rho \mapsto \sum_{j \in \Omega}\left(\Pi_{\mathscr{V}_{j}} \rho \Pi_{\mathscr{V}_{j}}, j\right)
$$

Это допустимое преобразование называется измерением квантовой переменной $z_{\Omega}$. (Комбинированнное пространство $\mathbf{L}(\mathscr{N}) \times \Omega$ соответствует объединению классической и квантовой подсистем; состояние классической подсистемы назьвается результатом измерения.) Измерение $\mathrm{MEAS}_{\Omega}$, примененное к состоянию $\rho \in \mathbf{D}(\mathscr{N})$, дает результат $j$ с вероятностью $\operatorname{Tr}\left(\Pi_{\mathscr{V}_{j}} \rho \Pi_{\mathscr{V}_{j}}\right)=\mathrm{P}\left(\rho, \mathscr{V}_{j}\right)$. При этом квантовая подсистема переходит в новое состояние $\rho^{\prime}=\mathrm{P}\left(\rho, \mathscr{V}_{j}\right)^{-1} \Pi_{\mathscr{V}_{j}} \rho \Pi_{\mathscr{V}_{j}}$. В физике такой процесс называется редукцией квантового состояния.

3.3. Точность. В теории квантовых вычислений (как и в реальной жизни) операторы, квантовые состояния и т.п. задаются с некоторой точностью. Таким образом, возникает необходимость в определении метрик на соответствующих пространствах. Определим сначала естественную метрику на пространстве вероятностных распределений.

Предположим, что некоторое устройство должно порождать случайную величину с вероятностным распределением $w(a): a \in \Gamma$. Если при работе этого устройства происходит ошибка с вероятностью $\leqslant \varepsilon$, то истинное распределение $\widetilde{w}$ удовлетворяет условию $\sum_{a \in \Gamma}|\widetilde{w}(a)-w(a)| \leqslant 2 \varepsilon$. Обратно, при вьполнении этого условия можно утверждать, что вероятность ошибки не превосходит $2 \varepsilon$. Таким образом, естественная метрика на пространстве распределений задается $\ell_{1}$-нормой $\|v\|_{1}=\sum_{a \in \Gamma}|v(a)|$. 
Точность вектора $|\xi\rangle \in \bar{\Gamma}$ характеризуется обычной (евклидовой) нормой $\| \xi\rangle \|=$ $\sqrt{\langle\xi \mid \xi\rangle}$. Сушествует две естественные нормы на пространстве линейных операторов $\mathbf{L}(\Gamma)$ : обычная операторная норма

$$
\|\mathrm{A}\|=\sup _{|\xi\rangle \neq 0} \frac{\| \mathrm{A}|\xi\rangle \|}{\| \xi\rangle \|}
$$

и двойственная к ней следовая норма

$$
\left.\|A\|_{1}=\sup _{B \neq 0} \frac{|\operatorname{Tr} A B|}{\|B\|}=\inf \left\{\sum_{j} \| \xi_{j}\right\rangle\|\|\left\langle\eta_{j} \|: \sum_{j} \mid \xi_{j}\right\rangle\left\langle\eta_{j}\right|=A\right\}=\operatorname{Tr} \sqrt{A^{\dagger} A}
$$

Норма $\|\cdot\|$ характеризует точность унитарных операторов, тогда как норма $\|\cdot\|_{1}$ подходит для смешанных состояний (поскольку она аналогична обычной $\ell_{1}$-норме, см. также предложение 3.8 ниже).

Имеется некоторая (по-видимому, неизбежная) несогласованность в определении норм чистых и смешанных состояний. При переходе от чистых состояний к смешанным точность сохраняется. Если $|\xi\rangle,|\eta\rangle$ - единичные векторы, то

$$
\||\xi\rangle\langle\xi|-| \eta\rangle\left\langle\eta\left|\left\|_{1}=2 \sqrt{1-|\langle\xi \mid \eta\rangle|^{2}} \leqslant 2\right\|\right| \xi\right\rangle-|\eta\rangle \| .
$$

Однако при обратном переходе (по формуле (13)) точность теряется. Если $\rho, \gamma \in$ $\mathbf{D}(\mathscr{N})$, то

$$
\|\sqrt{\rho}-\sqrt{\gamma}\|_{2} \leqslant \sqrt{\|\rho-\gamma\|_{1}}, \text { где }\|A\|_{2}=\sqrt{\operatorname{Tr} A^{\dagger} A} .
$$

(Норма $\|\cdot\|_{2}$ - это обычная евклидова норма в пространстве $\mathscr{N} \otimes \mathscr{N}^{*}$.) Улучшить эту оценку невозможно.

Нормы $\|\cdot\|$ и $\|\cdot\|_{1}$ обладают следуюшими основными свойствами:

$$
\|A B\| \leqslant\|A\|\|B\|, \quad\|A B\|_{1},\|B A\|_{1} \leqslant\|B\|\|A\|_{1}, \quad|\operatorname{Tr} A| \leqslant\|A\|_{1} .
$$

Они также хорошо себя ведут относительно тензорного умножения. Если $A \in \mathbf{L}(\mathscr{N})$, $B \in \mathbf{L}(\mathscr{M}), C \in \mathbf{L}(\mathscr{N} \otimes \mathscr{M})$, то

$$
\|A \otimes B\| \leqslant\|A\|\|B\|, \quad\|A \otimes B\|_{1} \leqslant\|A\|_{1}\|B\|_{1}, \quad\left\|\operatorname{Tr}_{\mathscr{M}} C\right\|_{1} \leqslant\|C\|_{1} .
$$

ПРЕДЛОЖЕНИЕ 3.8. Пусть $\Omega$ - набор взаимно ортогональных подпространств пространства $\mathscr{N}$. Тогда для любой пары смешанных состояний $\rho, \gamma$

$$
\sum_{\mathscr{V} \in \Omega}|\mathrm{P}(\rho, \mathscr{V})-\mathrm{P}(\gamma, \mathscr{V})| \leqslant\|\rho-\gamma\|_{1}
$$


ДоКАЗАТЕЛЬСТвО. Левую часть этого неравенства можно представить в виде $\operatorname{Tr}((\rho-\gamma) B)$, где $B=\sum_{\mathscr{V} \in \Omega}\left( \pm \Pi_{\mathscr{V}}\right)$. Ясно, что $\|B\| \leqslant 1$. Теперь используйте свойства норм (19).

Естественная норма на пространстве преобразований дается формулой

$$
\|T\|_{1}=\sup _{X \neq 0} \frac{\|T X\|_{1}}{\|X\|_{1}}=\sup _{X \neq 0} \frac{\left\|T^{*} X\right\|}{\|X\|} .
$$

$\mathrm{K}$ сожалению, эта норма неустойчива относительно тензорного умножения. Пример: рассмотрим преобразование $T:|i\rangle\langle j|\mapsto| j\rangle\langle i|(i, j=0,1)$. Очевидно, что $\|T\|_{1}=1$, однако $\left\|T \otimes I_{\mathscr{B}}\right\|_{1}=2$. (Подействуйте преобразованием $T \otimes I_{\mathscr{B}}$ на оператор $X=$ $\left.\sum_{i, j}|i, i\rangle\langle j, j|.\right)$ По этой причине мы будем использовать другую норму на пространстве преобразований $T(\mathscr{N}, \mathscr{M})$ :

$$
\|T\|_{\diamond}=\inf \left\{\|A\|\|B\|: \operatorname{Tr}_{\mathscr{F}}\left(A \cdot B^{\dagger}\right)=T\right\}, \quad A, B \in \mathbf{L}(\mathscr{N}, \mathscr{M} \otimes \mathscr{F}) .
$$

Здесь $\mathscr{F}$ - произвольное унитарное пространство размерности $\geqslant(\operatorname{dim} \mathscr{N})(\operatorname{dim} \mathscr{M})$. То, что это действительно норма, вытекает из следующего результата.

ПреДЛОЖЕНИЕ 3.9. $\|T\|_{\diamond}=\left\|T \otimes I_{\mathscr{G}}\right\|_{1} \geqslant\|T\|_{1}$, əде $\operatorname{dim} \mathscr{G} \geqslant \operatorname{dim} \mathscr{N}$.

ДокАЗАТЕЛЬСТво. Не очевидно лишь неравенство $\|T\|_{\diamond} \leqslant\|T \otimes I \mathscr{G}\|_{1}$. Без уменьшения обшности $\|T\|_{\diamond}=1$. Инфимум в формуле (21) достигается при $\|A\|=\|B\|=1$. Пусть $\mathscr{K}=\operatorname{Ker}\left(A^{\dagger} A-1_{\mathscr{N}}\right), \mathscr{L}=\operatorname{Ker}\left(B^{\dagger} B-1_{\mathscr{N}}\right)$,

$$
E=\left\{\operatorname{Tr}_{\mathscr{M}}\left(A \rho A^{\dagger}\right): \rho \in \mathbf{D}(\mathscr{K})\right\}, \quad F=\left\{\operatorname{Tr}_{\mathscr{M}}\left(B \gamma B^{\dagger}\right): \gamma \in \mathbf{D}(\mathscr{L})\right\} .
$$

Величина $\|A\|\|B\|$ минимальна по отношению к бесконечно мальм вариациям скалярного произведения $\delta\langle\cdot \mid \cdot\rangle=\langle\cdot|Z| \cdot\rangle$ на пространстве $\mathscr{F}$. Следовательно, не сушествует эрмитова оператора $Z \in \mathbf{L}(\mathscr{F})$ такого, что $\operatorname{Tr} X Z>\operatorname{Tr} Y Z$ для любых $X \in E, Y \in F$. Так как $E$ и $F$ - компактные выпуклые множества, $E \cap F \neq \varnothing$.

Пусть $\operatorname{Tr}_{\mathscr{M}}\left(A \rho A^{\dagger}\right)=\operatorname{Tr}_{\mathscr{M}}\left(B \gamma B^{\dagger}\right)$, где $\rho \in \mathbf{D}(\mathscr{K}), \gamma \in \mathbf{D}(\mathscr{L})$. Представим $\rho$ и $\gamma$ в виде $\rho=\operatorname{Tr} \mathscr{G}(|\xi\rangle\langle\xi|), \gamma=\operatorname{Tr} \mathscr{G}(|\eta\rangle\langle\eta|)$, где $|\xi\rangle,|\eta\rangle \in \mathscr{N} \otimes \mathscr{G}$ - единичные векторы. Положим $X=|\xi\rangle\langle\eta|$. Тогда $\left\|\left(T \otimes I_{\mathscr{G}}\right) X\right\|_{1}=\|X\|_{1}=1$.

Из формулы (21) и предложения 3.9 следует, что

$$
\|T \otimes R\|_{\diamond}=\|T\|_{\diamond}\|R\|_{\diamond}, \quad\|T R\|_{\diamond} \leqslant\|T\|_{\diamond}\|R\|_{\diamond} .
$$

Норма допустимого преобразования всегда равна единище. Если $V, W-$ унитарные вложения, то $\left\|V \cdot V^{\dagger}-W \cdot W^{\dagger}\right\|_{\diamond} \leqslant 2\|V-W\|$.

Лемма 3.10. Пусть $T, P \in \mathbf{T}(\mathscr{N}, \mathscr{M})$ - допустимье преобразования, $a V u W$ - их канонические представления. Тогда $\|V-W\| \leqslant(\operatorname{dim} \mathscr{M}) \sqrt{\|T-P\|_{\diamond}}$.

ДоКАЗАТЕЛЬСТво. Каждому преобразованию $R \in \mathbf{T}(\mathscr{N}, \mathscr{M})$ соответствует оператор $\breve{R}: \mathbf{L}(\mathscr{N}, \mathscr{M}) \rightarrow \mathbf{L}(\mathscr{N}, \mathscr{M})$, которому, в свою очередь, можно сопоставить оператор $\breve{R}^{\triangleright}: \mathscr{N} \rightarrow \mathscr{M} \otimes \mathscr{M}^{*} \otimes \mathscr{N}$ с компонентами $\left(\breve{R}^{\triangleright}\right)_{(i k l, j)}=R_{(i j k l)}$. В этих обозначениях $V=\left(\breve{T}^{1 / 2}\right)^{\triangleright}, W=\left(\breve{P}^{1 / 2}\right)^{\triangleright}$. Легко показать, что для любого $R \in \mathbf{T}(\mathscr{N}, \mathscr{M})$

$$
\|\breve{R}\| \leqslant(\operatorname{dim} \mathscr{M})\|R\|_{\diamond}, \quad\left\|\breve{R}^{\triangleright}\right\| \leqslant(\operatorname{dim} \mathscr{M})^{1 / 2}\|\breve{R}\| .
$$

Следовательно,

$$
\begin{aligned}
\|V-W\| & \leqslant(\operatorname{dim} \mathscr{M})^{1 / 2}\left\|\breve{T}^{1 / 2}-\breve{P}^{1 / 2}\right\| \\
& \leqslant(\operatorname{dim} \mathscr{M})^{1 / 2}\|\breve{T}-\breve{P}\|^{1 / 2} \leqslant(\operatorname{dim} \mathscr{M}) \sqrt{\|T-P\|_{\diamond}}
\end{aligned}
$$




\section{4. Квантовые модели вычислений}

4.1. Определения и основные свойства. Мы собираемся определить квантовую модель, которая аналогична необратимому (т.е. создающему мусор) классическому вычислению с обратимыми операциями. Обратимое квантовое вычисление будет определено позже. Прежде всего, мы обсудим, какие действия над квантовой системой можно реализовать физически. Начиная с этого места, мы рассматриваем системы с классическим множеством состояний $\Gamma=\mathbf{B}^{\Delta}=\mathbf{B}^{l}$ (где $\Delta=\{1, \ldots, l\}-$ множество битов). Соответствующее унитарное пространство имеет вид $\bar{\Gamma}=\mathscr{B} \otimes \Delta$.

Рассмотрим унитарный оператор $U \in \mathrm{U}\left(\mathscr{B}^{\otimes n}\right)$ и регистр $A \subseteq \Delta$ размера $n$. В этой ситуации $U[A]$ обозначает действие оператора $U$ на пространстве $\mathscr{B}{ }^{\otimes}$. Можно распространить это действие на полное пространство состояний $\mathscr{B} \otimes \Delta$, умножив его тензорно на тождественньй оператор $1[\Delta \backslash A] \in \mathrm{U}(\mathscr{B} \otimes(\Delta \backslash A))$. Как и в классическом случае, операторы вида $U[A] \in \mathscr{B}^{\otimes} \Delta$ назьваются операчиями. Физическая реализация таких операторов представляется возможной, если число $n$ мало.

Для каждого $y \in \mathbf{B}^{n}$ рассмотрим стандартное пространство

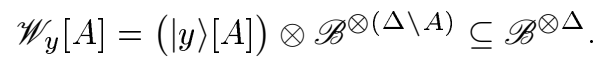

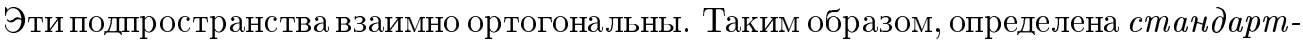
ная квантовая переменная $z_{A}$, принимающая значения из $\mathbf{B}^{n}$. Для данного состояния квантовой системы $|\xi\rangle$ можно измерить значение переменной $z_{A}$, т.е. организовать некоторую физическую процедуру, которая выдает результат $y$ с вероятностью $\mathrm{P}\left(|\xi\rangle, \mathscr{W}_{y}[A]\right)$. Измерение разрушает квантовое состояние, поэтому оно должно производиться в самом конще вычисления.

Перейдем к более формальному рассмотрению. Пусть память $\Delta$ состоит из входного регистра $X$ и рабочего регистра $W$; выходной регистр $Y \subseteq \Delta$ произволен (при этом $|X|=n,|Y|=m)$. Классические состояния памяти записываются в виде $(x, w)$, где $x \in \mathbf{B}^{X}, w \in \mathbf{B}^{W}$.

ОПРЕДЕЛЕНИЕ 4.1. Пусть $\mathscr{A}$ - некоторое множество унитарных операторов ( $6 a-$ зис), $0<\varepsilon<\frac{1}{2}-$ произвольная константа. Говорят, что квантовая схема (т.е. последовательность операций) $U_{1}\left[A_{1}\right], \ldots, U_{L}\left[A_{L}\right]\left(U_{i} \in \mathscr{A}\right)$ вычисляет функиию $F: N \rightarrow \mathbf{B}^{m}\left(N \subseteq \mathbf{B}^{n}\right)$ с вероятностью ошибки $\leqslant \varepsilon$, если

$$
\forall x \in N \quad \mathrm{P}\left(|\xi(x)\rangle, \mathscr{W}_{F(x)}[Y]\right) \geqslant 1-\varepsilon, \quad \text { где }|\xi(x)\rangle=U_{L}\left[A_{L}\right] \cdots U_{1}\left[A_{1}\right]|x, 0\rangle .
$$

Вероятность ошибки можно сделать сколь угодно малой, повторяя вычисление несколько раз. В самом деле, возьмем $k$ различных копий памяти и проделаем в каждой из них одно и то же вычисление с одним и тем же входным словом $x \in N$. Благодаря свойству (11) соответствующие выходные слова $y_{1}, \ldots, y_{k}$ можно рассматривать как независимые случайные переменные. Если более половины из всех $y_{i}$ совпадают и равны $y$, то это $у$ и берется в качестве окончательного результата. Данную “процедуру голосования" можно представить в виде формулы $y=\operatorname{MAJ}\left(y_{1}, \ldots, y_{k}\right)$, где МАJ - (частичная) функция, назьваемая функиией большинства. При такой организации вычисления полная вероятность ошибки не превосходит $\sum_{j \geqslant k / 2}\left(\begin{array}{c}k \\ j\end{array}\right) \varepsilon^{j}(1-\varepsilon)^{k-j} \leqslant \lambda^{k}$, где $\lambda=2(\varepsilon(1-\varepsilon))^{1 / 2}<1$. Таким образом, ценой полиномиального увеличения размера схемы можно уменьшить вероятность ошибки до $\exp (-p(n))$, где $p-$ произвольная 
функция полиномиального роста. При этом первоначальный выбор константы $\varepsilon$ не важен; обычно полагают, что $\varepsilon=\frac{1}{3}$. Замечание: Функция МАЈ вычисляется булевой схемой размера $O(k \log k)$. Однако, чтобы оставаться в рамках определения 4.1, следует представить оператор $\widehat{\mathrm{MAJ}}_{\oplus}$ в базисе $\mathscr{A}$. Это возможно, например, в полном квантовом базисе $\mathscr{Q}$, см. ниже.

ПРЕДЛОЖЕНИЕ 4.2. Пусть квантовая схема размера L вьиисляет функцию $F$ с вероятностью ошибки $\leqslant \varepsilon$. Если каждая операция этой схемы выполняется $c$ точностью $\delta$, то результирующая вероятность ошибки не превосходит $\varepsilon+2 L \delta$. (Таким образом, точность $\delta<$ const $L^{-1}$ достаточна для надежной работь cхемьн.)

ДокАЗАТЕЛЬСтво. Пусть $U_{1}, \ldots, U_{L}, \widetilde{U}_{1}, \ldots, \widetilde{U}_{L}-$ унитарные операторы. Если $\left\|\widetilde{U}_{j}-U_{j}\right\| \leqslant \delta$, то $\left\|\widetilde{U}_{L} \cdots \widetilde{U}_{1}-U_{L} \cdots U_{1}\right\| \leqslant L \delta$. Теперь воспользуемся неравенством (18) и предложением 3.8 .

Выбор базиса. В этой статье используется стандартный базис $\mathscr{Q}=\left\{1_{\mathscr{B}}, S, K\right.$, $\left.\widehat{\oplus}, \widehat{\wedge}_{\oplus}\right\}$, где

$$
S=\frac{1}{\sqrt{2}}\left(\begin{array}{cc}
1 & 1 \\
1 & -1
\end{array}\right), \quad K=\left(\begin{array}{ll}
1 & 0 \\
0 & \mathrm{i}
\end{array}\right)
$$

Если имеется оракул $F$, мы добавляем к базису оператор $\widehat{F}_{\oplus} \cdot{ }^{7}$ Мы увидим, что базис $\mathscr{Q}$ полон в приближенном смысле, см. теорему 4.8 ниже. (Грубо говоря, он порождает всюду плотное множество унитарных операторов.) По поводу полноты некоторых других базисов см. [53].

Моделирование классической вероятности. (Сравните с примером 3.3.) Чтобы моделировать классическое вероятностное вычисление, необходимо уметь создавать случайные биты. Возьмем квантовьй бит в состоянии $S|0\rangle=2^{-1 / 2}(|0\rangle+|1\rangle)$ и скопируем его в другой кубит при помоши оператора $\widehat{\oplus}$. (Предостережение: оператор (Ф) копирует каждое базисное состояние, входящее в квантовую суперпозищию, а не всю суперпозицию целиком!) Таким способом мы получаем двухбитное квантовое состояние $|\psi\rangle=2^{-1 / 2}(|0,0\rangle+|1,1\rangle)$. Теперь уничтожим одну из копий (или просто не будем использовать ее в дальнейшем). Эта ситуация описьвается как переход к оператору плотности оставшегося кубита

$$
\rho^{\prime}=\operatorname{Tr}_{2}(|\psi\rangle\langle\psi|)=\left(\begin{array}{cc}
1 / 2 & 0 \\
0 & 1 / 2
\end{array}\right) .
$$

Этот оператор плотности соответствует классической вероятностной мере $\mu(0)=$ $\mu(1)=\frac{1}{2}$.

Обратимое квантовое вычисление. Определение 2.2 непосредственно обобщается на квантовьй случай. С технической точки зрения полезно перефформулировать это определение в терминах частичных операторов. Частичный унитарный оператор на пространстве $\mathscr{N}$ - это унитарный оператор $U: \mathscr{L} \rightarrow \mathscr{M}$, где $\mathscr{L}, \mathscr{M} \subseteq$

\footnotetext{
${ }^{7}$ Если $F-$ частичная функция, то оператор $\widehat{F_{\oplus}}$ тоже частичньй. (Частичньй унитарньй оператор - это унитарное линейное отображение одного подпространства в другое.)
} 
$\mathscr{N}$. Например, $\tau:|0\rangle \mapsto|0\rangle-$ это частичньй унитарный оператор на пространстве $\mathscr{B} \otimes k$ ( $k$ - любое). Множество частичных унитарных операторов на $\mathscr{N}$ обозначается через $\check{\mathbf{U}}(\mathscr{N})$.

Понятие точности применимо к частичным унитарным операторам. Пусть $U: \mathscr{L} \rightarrow \mathscr{M}$ и $\widetilde{U}: \widetilde{\mathscr{L}} \rightarrow \widetilde{\mathscr{M}}$ - частичные унитарные операторы на пространстве $\mathscr{N}$. Мы будем говорить, что $\widetilde{U}$ представляет $U$ с точностью $\delta$, если $\mathscr{L} \subseteq \widetilde{\mathscr{L}}, \mathscr{M} \subseteq \widetilde{\mathscr{M}}$ и $\|(\widetilde{U}-U)|\xi\rangle\|\leqslant \delta\| \xi\rangle \|$ для любого $|\xi\rangle \in \mathscr{L}$. Заметим, что $\widetilde{U}^{-1}$ представляет $U^{-1} \mathrm{c}$ той же точностью.

Пусть $\Delta=X \cup W$ - память (множество кубитов); $|X|=n, X \cap W=\varnothing$.

ОПРЕДЕЛЕНИЕ 4.3. Говорят, что квантовая схема (т.е. последовательность операций) $U_{1}\left[A_{1}\right], \ldots, U_{L}\left[A_{L}\right]$ представляет оператор $U \in \check{\mathbf{U}}\left(\mathscr{B}^{\otimes n}\right)$ (с точностью $\left.\delta\right)$, если произведение $U_{L}\left[A_{L}\right] \cdots U_{1}\left[A_{1}\right]$ представляет частичньй оператор $U[X] \otimes \tau[W]$ (с точностью $\delta$ ).

Таким образом, квантовая схема представляет оператор, но вичисляет булеву функцию.

Упражнение. (Сравните с предложением 2.4.) Пусть функции $G$ и $G^{-1}$ вьгисляются известными квантовыми схемами $\Psi, \Psi^{\prime}$ с вероятностью ошибки $\leqslant \frac{1}{3}$. Представьте оператор $U=\widehat{G}$ с произвольной точностью $\delta>0$. (Методы из раздела 2.2 работают в квантовом случае, нужно просто достаточно аккуратно обходиться с точностью и вероятностью ошибки.)

Влияние мусора. В классической постановке проблема обратимости вьглядит довольно искусственной. Мусор, если он и возникает при некоторых операциях, можно просто игнорировать - результат будет тот же, что и при обратимом вычислении. В квантовом случае это не так. Пусть $G: \mathbf{B}^{n} \rightarrow \mathbf{B}^{n}-$ биекция. Предположим, что квантовая схема использует оператор $\widehat{G}$ в качестве подпрограммы. Что произойдет, если мы заменим $\widehat{G}$ на другой классический оператор $V:|x, 0\rangle \mapsto|G(x), g(x)\rangle$ (где $g(x)$ - мусор)? Оператор $V$ преобразует квантовое состояние $|\xi\rangle=\sum_{x} c_{x}|x\rangle$ в состояние $|\psi\rangle=\sum_{x} c_{x}|G(x), g(x)\rangle$. Поскольку мусор игнорируется, нужно вычислить частичньй след по второй переменной. Таким образом, мы получаем смешанное состояние

$$
\rho^{\prime}=\sum_{x, y: g(x)=g(y)} c_{x}^{\star} c_{y}|G(x)\rangle\langle G(y)|,
$$

которое, разумеется, отличается от “правильного" состояния $\rho=G|\xi\rangle\langle\xi| G^{\dagger}$. В действительности, $\rho=\rho^{\prime}$ (для всех $|\xi\rangle$ ) только тогда, когда мусор $g(x)$ не зависит от $x$.

4.2. Построение различных операторов из элементов базиса. Операторы $S$ и $K$ (см. (24)) порождают конечную группу $E \subseteq \mathbf{U}(\mathscr{B})$ порядка 192. Эта група содержит матрицы Паули (7) (например, $\sigma_{x}=\hat{ᄀ}=S K^{2} S$ ) и постоянный оператор $\zeta=(S K)^{3}=\frac{1+\mathrm{i}}{\sqrt{2}}$. Факторизуя группу $E$ по ее центру $\left\{\zeta^{k}: k=0, \ldots, 7\right\}$, мы получаем соответствующую группу унитарных преобразований $\operatorname{ESp}_{2}(1) \subseteq \mathbf{U T}(\mathscr{B})$ порядка $24 .^{8}$ Группа $\mathrm{ESp}_{2}(1)$ изоморфна группе собственных врашений куба, причем

\footnotetext{
${ }^{8}$ Смысл обозначения $\mathrm{ESp}_{2}(1)$ статет понятен в разделе 9.1.
} 
матрицы Паули $\sigma_{x}, \sigma_{y}, \sigma_{z} \in \mathbf{L}\left(\mathscr{B}^{\otimes n}\right)$ преобразуются как базисные орты. Преобразования $S \cdot S^{\dagger}$ и $K \cdot K^{\dagger}$ действуют на матрицах Паули следуюшим образом:

$$
S \cdot S^{\dagger}:\left(\sigma_{x}, \sigma_{y}, \sigma_{z}\right) \mapsto\left(\sigma_{z},-\sigma_{y}, \sigma_{x}\right), \quad K \cdot K^{\dagger}:\left(\sigma_{x}, \sigma_{y}, \sigma_{z}\right) \mapsto\left(\sigma_{y},-\sigma_{x}, \sigma_{z}\right)
$$

Любой классический оператор можно представить в базисе $\widehat{\mathscr{R}}=\left\{1_{\mathscr{B}}, \sigma_{x}, \widehat{\oplus}, \widehat{\wedge}_{\oplus}\right\}$. (Это следует из полноты классического обратимого базиса $\mathscr{R}$.) Следовательно, любой классический оператор представим в базисе $\mathscr{Q}$.

Теперь мы опишем одну очень полезную конструкцию - операторы с квантовым управлением. Пусть $U$ - частичный унитарньй оператор на $\mathscr{B} \otimes n$, т.е. $U: \mathscr{N} \rightarrow \mathscr{M}$ $\left(\mathscr{N}, \mathscr{M} \subseteq \mathscr{B}^{\otimes n}\right)$. Определим новый оператор $\Lambda(U): \mathscr{B} \otimes \mathscr{N} \rightarrow \mathscr{B} \otimes \mathscr{M}$ при помоши формулы

$$
\Lambda(U)|a, \xi\rangle= \begin{cases}|0, \xi\rangle, & \text { если } a=0, \\ |1\rangle \otimes U|\xi\rangle, & \text { если } a=1 .\end{cases}
$$

Эта формула означает, что оператор $U$ применяется или не применяется в зависимости от того, чему равен дополнительный управляющий бит (1 или 0). В этом определении управляюший бит - квантовьй. Оператор с классическим управлением $\Lambda_{c}(U)$ будет определен в разделе 7 (точнее говоря, это будет преобразование, а не оператор). Разница состоит в том, что оператор $\Lambda(U)$ чувствует фазовьй множитель между различными состояниями управляюшего бита. Значение этого свойства станет понятно из "Важного примера", см. раздел 5.

Примеры: $\Lambda\left(\sigma_{x}\right)=\widehat{\oplus}, \Lambda(\widehat{\oplus})=\widehat{\wedge}_{\oplus}, \Lambda(\mathrm{i})=K$. (В последнем случае число і рассматривается как оператор на $\mathbf{C}=\mathscr{B} \otimes 0$.) Для построения более сложных операторов вида $\Lambda(U)$ можно использовать следуюшие тождества:

$$
\Lambda(U V)=\Lambda(U) \Lambda(V), \quad \Lambda\left(V^{-1} U V\right)[1, A]=V^{-1}[A] \Lambda(U)[1, A] V[A]
$$

ПримеР 4.4 (Fredkin's gate).

$$
\Lambda(\leftrightarrow): \mathscr{B}^{\otimes 3} \rightarrow \mathscr{B}^{\otimes 3}: \quad|a, b, c\rangle \mapsto\left\{\begin{array}{l}
|0, b, c\rangle, \text { если } a=0, \\
|1, c, b\rangle, \text { если } a=1 .
\end{array}\right.
$$

Этот оператор можно представить следующим образом (ср. с (4)):

$$
\Lambda(\leftrightarrow)[1,2,3]=\widehat{\wedge}_{\oplus}[1,2,3] \widehat{\wedge}_{\oplus}[1,3,2] \widehat{\wedge}_{\oplus}[1,2,3]
$$

ЛЕмма 4.5. Пусть $U$ - (частичный) унитарный оператор на $\mathscr{B}{ }^{\otimes n}$ такой, что $U|0\rangle=|0\rangle$. Тогда оператор $\Lambda(U)$ можсно представить квантовой схемой размера $6 n+1$ в базисе $\mathscr{Q} \cup\{U\}$. Элемент $U$ входит в эту схему один раз. 
ДокАЗАТЕЛьство. Пусть $A$ и $B$ - два непересекающихся регистра размера $n$. Частичньй оператор $\Lambda(U)[1, A] \otimes \tau[B]$ представляется как

$$
\Lambda(\leftrightarrow)[1, A, B] \quad U[A] \quad \Lambda(\leftrightarrow)[1, A, B] .
$$

Используя эту лемму, мы можем представить все операторы $\Lambda(U): U \in \mathscr{Q}$, кроме $\Lambda(S)$. К сожалению, оператор $\Lambda(S)$ может быть представлен лишш приближенно, что создает некоторые технические неудобства. Ситуация упрощается, если заменить базис $\mathscr{Q}$ на другой базис

$$
\mathscr{Q}^{\prime}=\left\{1_{\mathscr{B}}, S^{\prime}, K, \widehat{\oplus}, \widehat{\wedge}_{\oplus}\right\}, \text { где } S^{\prime}=\frac{1+\mathrm{i}}{\sqrt{2}} S .
$$

Заметим, что $S^{\prime}=K\left(S^{\prime}\right)^{-1} K S^{\prime} K,\left(S^{\prime}\right)^{-1}=\left(S^{\prime}\right)^{7}$, поэтому $\Lambda\left(S^{\prime}\right)=\Lambda(K)\left(S^{\prime}\right)^{7} \times$ $\Lambda(K) S^{\prime} \Lambda(K)$. Базис $\mathscr{Q}^{\prime}$ порождает множество операторов, замкнутое относительно преобразования $U \mapsto \Lambda(U)$ (а также $U \mapsto U^{-1}$ ). Элементы базиса $\mathscr{Q}^{\prime}$ - рациональные числа со знаменателями вида $2^{r}$. С другой стороны, $\mathscr{Q}$ и $\mathscr{Q}^{\prime}$ порождают одно и то же множество унитарных преобразований $U \cdot U^{\dagger}$; соответствуюшие операторы $U$ отличаются лиш фазовыми множителями. Фазовые множители не важны для физически осмысленного определения 4.1; они имеют значение только для технического определения 4.3. Кроме того, каждый элемент $\mathscr{Q}^{\prime}$ может быть представлен в базисе $\mathscr{Q}$.

Мы собираемся доказать, что базис $\mathscr{Q}^{\prime}$ (а следовательно, и $\mathscr{Q}$ ) полон в приближенном смысле. Полнота базиса $\mathscr{Q}^{\prime}$ следует из полноты бесконечного базиса $\mathscr{Q}^{\prime} \cup \mathbf{U}(1)$. Чтобы представить постоянньй оператор $c \in \mathbf{U}(1)$ в базисе $\mathscr{Q}^{\prime}$, достаточно представить оператор $\Lambda(c) \in \mathbf{U}(\mathscr{B})$ с точностью до фазового множителя. В самом деле,

$$
\sigma_{x} \Lambda(\mathrm{c}) \sigma_{x} \Lambda(\mathrm{c})^{-1}:|0\rangle \mapsto c|0\rangle .
$$

Лемма 4.6. Onераторы $S[1], S[2]$ u $\Lambda(K)[1,2]=\Lambda(K)[2,1]$, вместе со всевозможнимм фазовыми множителями, порождают плотное подмножество в 2pynne $\mathbf{U}\left(\mathscr{B}^{\otimes 2}\right)$.

ДОКАЗАТЕЛЬСТвО. Пусть $X=\Lambda(S K S), X_{1}=X[1,2], X_{2}=X[2,1]$. Операторы $X_{1}, X_{2}$ сохраняют векторы $|00\rangle$ и $|\eta\rangle=|01\rangle+|10\rangle+|11\rangle$. Обозначим через $\mathscr{L}$ двумерное подпространство, ортогональное к этим векторам. Ясно, что $X_{1}, X_{2} \in \mathbf{U}(\mathscr{L})$. Более того, операторы $Y_{1}=X_{1} X_{2}^{-1}$ и $Y_{2}=X_{2}^{-1} X_{1}$ принадлежат групе $\operatorname{SU}(\mathscr{L})$. Эти операторы не коммутируют друг с другом и имеют собственные значения $\frac{1}{4}(1 \pm$ $\sqrt{-15})$, не являющиеся корнями из единицы. Отсюда вытекает, что $Y_{1}$ и $Y_{2}$ порождают плотное подмножество в $\mathbf{S U}(\mathscr{L})$.

Теперь мы воспользуемся следующим геометрическим фактом. Пусть $\mathscr{M}-$ унитарное пространство размерности $\geqslant 3$. Рассмотрим подгруппу $H \subset \mathbf{S U}(\mathscr{M})$ - стабилизатор некоторого ненулевого вектора $|\xi\rangle \in \mathscr{M}$. Пусть $V$ - произвольный унитарный оператор, не сохраняющий подпространство $(|\xi\rangle)$. Тогда множество операторов $H \cup V^{-1} H V$ порождает всю группу $\mathbf{S U}(\mathscr{M})$.

Унитарный оператор $V=\Lambda(K)$ сохраняет вектор $|00\rangle$, но не сохраняет подпространство $(|\eta\rangle)$. Следовательно, $Y_{1}, Y_{2}, V^{-1} Y_{1} V, V^{-1} Y_{2} V$ порождают плотное подмножество в $\mathbf{S U}(\mathscr{L} \oplus(|\eta\rangle))$. Наконец, оператор $S[1]$ не сохраняет подпространство $(|00\rangle)$, следовательно, можно породить плотное подмножество в $\mathbf{S U}\left(\mathscr{B}^{\otimes 2}\right)$. 
Этот результат неконструктивен. Пока непонятно, как приближать алгоритмически данную унитарную матрищу $U$ произведениями операторов $S[1], S[2]$ и $\Lambda(K)$. Для этого нам потребуется следующая лемма, которую независимо доказали R. Solovay [54] и автор.

Лемма 4.7. Пусть матрицы $X_{1}, \ldots, X_{r} \in \mathbf{S U}(n)$ порождают плотное подмножество в $\mathbf{S U}(n)$. Существует алгоритм, позволяющий приблизить данную матрицу $U \in \mathbf{S U}(n)$ произведением матрии $X_{1}, \ldots, X_{r}, X_{1}^{-1}, \ldots, X_{r}^{-1}$ с заданной точностью $\delta>0$. Этот алгоритм полиномиален по $\log (1 / \delta)$.

НАБРОсОК ДОКАЗАТЕЛЬСтВА. Алгоритм состоит из двух частей, первая из которых не зависит от $U$. Сначала мы опишем вторую, тривиальную часть алгоритма.

$(R, \varepsilon)$-сеть - это конечное множество точек в $\mathbf{S U}(n)$ такое, что $\varepsilon$-окрестности этих точек покрьвают $R$-окрестность единичного элемента. Пусть $a>2$ и $b>0$ - некоторые константы. Допустим, что мы уже построили некоторую $(\infty, b)$-сеть $\Gamma_{0}$ и $\left(a b 2^{-k}, b 2^{-k}\right)$-сети $\Gamma_{k}\left(1 \leqslant k \leqslant l=\left\lceil\log _{2}(a b / \delta)\right\rceil\right)$. Для любой матрицы $U \in \mathbf{S U}(n)$ можно найти такие $A_{k} \in \Gamma_{k}(k=0, \ldots, l)$, что $A_{0} A_{1} \cdots A_{k}$ принадлежит $c b 2^{-k}$-окрестности $U$ (для каждого $k$ ). Здесь $1<c<a / 2$-произвольная константа (поправка на неточность вычислений с вещественньми числами).

Чтобы построить сети $\Gamma_{0}$ и $\Gamma_{1}$, нужно просто породить достаточно много различных произведений из матриц $X_{1}, \ldots, X_{r}, X_{1}^{-1}, \ldots, X_{r}^{-1}$. Этот шаг требует фиксированного числа операций. Остальные сети строятся из $\Gamma_{1}$ с использованием матричных коммутаторов.

Выберем параметры $a>2 b>0$ и $s$ так, чтобы было вьполнено следуюшее свойство: для любых $\varepsilon^{\prime}, \varepsilon^{\prime \prime}<b$, для любой $\left(a \varepsilon^{\prime}, \varepsilon^{\prime}\right)$-сети $\Gamma^{\prime}$ и $\left(a \varepsilon^{\prime \prime}, \varepsilon^{\prime \prime}\right)$-сети $\Gamma^{\prime \prime}$ множество

$$
\left[\Gamma, \Gamma^{\prime}\right]^{s}=\left\{V_{1} \cdots V_{s}: V_{j}=A_{j} B_{j} A_{j}^{-1} B_{j}^{-1}, A_{j} \in \Gamma^{\prime}, B_{j} \in \Gamma^{\prime \prime}\right\}
$$

явлется $(a \varepsilon, \varepsilon / 2)$-сетью, где $\varepsilon=\varepsilon^{\prime} \varepsilon^{\prime \prime} / b$.

Сеть $\left[\Gamma, \Gamma^{\prime}\right]^{s}$ легко преобразуется в $(a \varepsilon, \varepsilon)$-сеть $\left[\Gamma, \Gamma^{\prime}\right]_{\star}^{s}$ ограниченной мошности. В самом деле, любая $(R, \varepsilon / 2)$-сеть $\Gamma$ содержит $(R, \varepsilon)$-сеть $\Gamma_{\star}$ с дополнительными свойствами:

(a) расстояние между любыми двумя точками из $\Gamma_{\star}$ больше, чем $\varepsilon / 3$;

(б) $\Gamma_{\star}$ содержится в $(R+\varepsilon)$-окрестности единичного элемента.

Очевидно, что $\left|\Gamma_{\star}\right| \leqslant O(R / \varepsilon)^{n^{2}-1}$ (поскольку $\mathbf{S U}(n)$ является многообразием размерности $\left.n^{2}-1\right)$. Такое подмножество $\Gamma_{\star}$ можно выбрать из Г посредством эффективной процедуры.

Таким образом, сети $\Gamma_{k}(k=2, \ldots, l)$ можно построить согласно следуюшим рекурсивным правилам: $\Gamma_{2 k}=\left[\Gamma_{k}, \Gamma_{k}\right]_{\star}^{s}, \Gamma_{2 k+1}=\left[\Gamma_{k}, \Gamma_{k+1}\right]_{\star}^{s}$. Каждьй элемент этих сетей является произведением $O\left((\log (1 / \delta))^{\log _{2}(4 s)}\right)$ матриц из множества $\left\{X_{1}, \ldots, X_{r}, X_{1}^{-1}, \ldots, X_{r}^{-1}\right\}$. Отсюда следует, что алгоритм полиномиален по $\log (1 / \delta)$.

Используя эти результаты, мы можем представить каждый оператор $V \in \mathbf{U}(\mathscr{B})$ и $\Lambda(V) \in \mathbf{U}\left(\mathscr{B}^{\otimes 2}\right)$ с точностью $\delta$ схемой размера poly $(\log (1 / \delta))$ в базисе $\mathscr{Q}^{\prime} \cup \mathbf{U}(1)$. Следовательно, оператор $\Lambda^{n-1}(V)=\Lambda(\Lambda(\ldots V \ldots))$ можно представить схемой размера $O(n)+$ poly $(\log (1 / \delta))($ согласно лемме 4.5). Этот оператор сохраняет все базисные векторы, кроме $|1 \ldots 1,0\rangle$ и $|1 \ldots 1,1\rangle$. Оператор вида $\widehat{G}^{-1} \Lambda^{n-1}(V) \widehat{G}$ (где $\left.G: \mathbf{B}^{n} \rightarrow \mathbf{B}^{n}\right)$ 
действует на произвольную пару базисных векторов. Любой унитарньй оператор $U \in \mathbf{U}\left(\mathscr{B}^{\otimes n}\right)$ можно представить в виде произведения $2^{n}\left(2^{n}-1\right) / 2$ таких операторов. Итак, мы получили следующий результат.

Теорема 4.8. Любую унитарную матрицу $U \in \mathrm{U}\left(\mathscr{B}^{\otimes n}\right)$ можно представить, $c$ произвольной точностью $\delta>0$, квантовой схемой размера $\exp (O(n))$ poly $(\log (1 / \delta))$ в базисе $\mathscr{Q}^{\prime}$. Эта схема строится алгоритмически за время $\exp (O(n))$ poly $(\log (1 / \delta))$.

4.3. Обобщенное квантовое управление и универсальные схемы. Цель этого раздела - дать читателю некоторое ощущение “квантового программирования". Суть проблемы состоит в следуюшем. Допустим, что при решении вычислительной задачи $A$ возникает подзадача $B$, для которой уже известен алгоритм. В классическом случае можно просто вызвать процедуру, решающую задачу $B$. А что такое вызов процедуры в квантовом случае? В простейшем понимании это просто подстановка одной схемы в другую, однако такое определение не обеспечивает достаточной гибкости. Полученные ниже результаты дают гораздо большую свободу действий и оправдьвают использование неформального процедурного язька. Впрочем, из этих результатов в дальнейшем понадобится лишш лемма 4.9.

Прежде всего, мы расширим понятие оператора с квантовым управлением. Общий вид квантового управления - это выбор одного оператора из некоторого семейства. Рассмотрим параметрическое семейство (частичных) унитарных операторов, т.е. функцию вида $\mathscr{U}: \mathbf{B}^{l} \rightarrow \check{\mathbf{U}}\left(\mathbf{B}^{n}\right)$. Определим оператор

$$
\Lambda(\mathscr{U}) \in \check{\mathbf{U}}\left(\mathscr{B}^{\otimes(l+n)}\right), \quad \Lambda(\mathscr{U}):|a, \xi\rangle \mapsto|a\rangle \otimes \mathscr{U}(a)|\xi\rangle .
$$

Благодаря следующей лемме можно легко перейти к подсемейству или другой параметризации того же семейства.

Лемма 4.9. Пусть $f: \mathbf{B}^{k} \rightarrow \mathbf{B}^{l}-$ (частичная) функиия, а $\mathscr{U}: \mathbf{B}^{l} \rightarrow \mathbf{\mathbf { U }}\left(\mathscr{B}^{\otimes n}\right)$ - параметрическое семейство (частичных) унитарных операторов. Рассмотрим другое параметрическое семейство $\mathscr{U} \circ f: \mathbf{B}^{k} \rightarrow \check{\mathbf{U}}\left(\mathscr{B}^{\otimes n}\right): a \mapsto \mathscr{U}(f(a))$. Предположим, что функиия $f$ может бить вычислена булевой схемой размера $L$ в стандартном базисе $\mathscr{C}$. Тогда оператор $\Lambda(\mathscr{U} \circ f)$ можнно представить квантовой схемой размера $2 L+1$ в базисе $\widehat{\mathscr{R}} \cup\{\Lambda(\mathscr{U})\}$, причем әлемент $\Lambda(\mathscr{U})$ будет входить в эту схему только один раз.

(Доказательство полностью аналогично доказательству предложения 2.3.)

ПримеР 4.10. Рассмотрим семейство унитарных матриц размера $2 \times 2$

$$
\mathscr{T}_{\delta}: \begin{aligned}
& \text { двоичное представление } \\
& \text { комплексных чисел } a, b, c, d \\
& \text { с точностью } \delta
\end{aligned} \mapsto \quad \text { оператор }\left(\begin{array}{ll}
a & b \\
c & d
\end{array}\right)
$$

Оператор $\Lambda\left(\mathscr{T}_{\delta}\right)$ позволяет подействовать на кубит любым унитарным оператором $U \in \mathbf{U}(\mathscr{B})$ по желанию (точнее говоря, “по квантовому желанию”). Мы увидим, что оператор $\Lambda\left(\mathscr{T}_{\delta}\right)$ можно представить в базисе $\mathscr{Q}$ с произвольной точностью. 
ПРИМЕР 4.11. Пусть $\Gamma_{n}=\left\{0, \ldots, 2^{n}-1\right\}$ (это множество естественно отождествить с $\left.\mathbf{B}^{n}\right)$. Допустим нам нужно создать квантовое состояние

$$
\left|\psi_{n}(q)\right\rangle=q^{-1 / 2} \sum_{j=0}^{q-1}|j\rangle \in \overline{\Gamma_{n}}=\mathscr{B}^{\otimes n}, \quad \text { где } q \in\left\{1, \ldots, 2^{n}\right\} .
$$

Это можно сделать при помоши следующей рекурсивной процедуры:

1. Пусть $q_{0}=\min \left\{2^{n-1}, q\right\}, q_{1}=q-q_{0}$.

2. Приведем первый бит в состояние $\left(q_{0} / q\right)^{1 / 2}|0\rangle+\left(q_{1} / q\right)^{1 / 2}|1\rangle$.

3 . В зависимости от значения $x$ этого бита создадим состояние $\left|\psi_{n-1}\left(q_{x}\right)\right\rangle$ в оставшихся $n-1$ битах.

Процедура заканчивается, когда $n=0$. Чтобы описать эту процедуру более строго, определим функции

$$
a_{n}: q \mapsto\left(q_{0} / q\right)^{1 / 2}, \quad b_{n}: q \mapsto\left(q_{1} / q\right)^{1 / 2}, \quad f_{n}:(q, x) \mapsto q_{x},
$$

где $q_{0}=\min \left\{2^{n-1}, q\right\}, q_{1}=q-q_{0}$. Введем также семейство частичных унитарных операторов $V_{n}(q):|0\rangle \mapsto\left|\psi_{n}(q)\right\rangle$. Тогда

$$
\Lambda\left(V_{n}\right)[A, 1, \ldots, n]=\Lambda\left(V_{n-1} \circ f_{n}\right)[A, 1, \ldots, n] \Lambda\left(\mathscr{T} \circ\left(a_{n}, b_{n}, b_{n},-a_{n}\right)\right)[A, 1],
$$

где $A$ - управляюший регистр, содержаший $q$. (Семейство операторов $\mathscr{T}$ было определено в предыдушем примере. Параметр точности $\delta$ для простоты опушен.)

Замечательным результатом классической теории алгоритмов является существование универсальной машины Тьюринга. Аналогичное (но, конечно, менее глубокое) свойство имеет место и для булевых схем. Мы собираемся построить универсальную квантовую схему, которая может моделировать любую схему заданного размера $L$ в базисе $\mathscr{Q}^{\prime}$. Очевидно, что схемы размера $L$ естественным образом кодируются двоичными словами длины $O(L \log L)$. Пусть

$$
\mathscr{U}_{L}: \quad \text { описание схемы размера } L \mapsto \text { оператор, представленньй этой схемой }
$$

Теорема 4.12. Oператоры $\Lambda\left(\mathscr{U}_{L}\right), L=1,2, \ldots$, могут быть представлень однородной последовательностью полиномиальных схем $\Psi_{L}$ в базисе $\mathscr{Q}^{\prime}$.

ДокАЗАТЕльство. Пусть $\Delta_{0}=\{1, \ldots, 3 L\}$. (3L - это наибольшее число битов, используемых в схеме размера $L$.) Применим операторы $\Lambda(U): U \in \mathscr{Q}^{\prime}$ (представленные в том же базисе $\left.\mathscr{Q}^{\prime}\right)$ ко всем возможным комбинациям битов из $\Delta_{0} \cdot{ }^{9}$ Повторим эту процедуру $L$ раз. Таким образом, мы построили схему, которая может моделировать любую схему размера $L$, если приписать соответствующие значения управляющим битам. Обозначим через $f$ функцию, которая преобразует описание схемы в значения управляюших битов. Теперь воспользуемся леммой 4.9 .

ЗАмЕчАНИЕ. Если бы мы заменили базис $\mathscr{Q}^{\prime}$ на $\mathscr{Q}$, мы смогли бы получить лишь приближенное представление.

СлЕДСТВИЕ. Оператор $\Lambda\left(\mathscr{T}_{\delta}\right)$ (см. пример 4.10) можно представить с точностью ${ }^{10} O(\delta)$ схемой размера poly $(\log (1 / \delta))$ в базисе $\mathscr{Q} ;$ эта схема строится алгоритмически за время poly $(\log (1 / \delta))$.

ДОКАЗАТЕЛЬСТВО: используйте теорему 4.8 и лемму 4.9.

\footnotetext{
${ }^{9}$ Соответствующие управляющие биты различны и не принадлежат $\Delta_{0}$.

10 Точность матрицы отличается от точности ее элементов.
} 


\section{5. Измеряющие операторы}

Мы только что видели, как многие классические конструкции переносятся в теорию квантовых вычислений. Теперь мы продемонстрируем некоторые принципиально новые возможности, которые в классическом случае отсутствуют.

Важньм физическим предположением, лежашим в основе определения 4.1, является возможность измерить классическое состояние памяти в конце вычисления. Более обшие квантовые измерения, производимые в процессе вычисления, могли бы быть очень полезным вычислительньм инструментом. К сожалению, стандартное определение измерения (см. пример 3.7) опирается на понятие комбинированной (квантово-классической) системы, которое выходит за рамки нашей вычислительной модели. Можно, однако, определить некоторый специальный класс унитарных операторов, которые действуют почти так же, как преобразования $\mathrm{MEAS}_{\Omega}$ из примера 3.7.

ОПРЕДЕЛЕНИЕ 5.1. Пусть $A$ и $D$ - два непересекаюшихся регистра, $C \subseteq D$, $|C|=m$. Пусть также $\left\{\mathscr{V}_{a}: a \in \Omega\right\}$ - семейство взаимно ортогональных подпространств пространства $\mathscr{B}^{\otimes A}$. Положим $\mathscr{N}=\bigoplus_{a \in \Omega} \mathscr{V}_{a} . \quad$ Для каждого $y \in \mathbf{B}^{m}$ обозначим через $\mathscr{W}_{y}=\mathscr{W}_{y}[C]$ соответствующее стандартное подпространство в $\mathscr{B} \otimes D$ (см. (23)). Измеряющий оператор для квантовой переменной $z_{\Omega}$ - это унитарный оператор вида

$$
U=\sum_{a \in \Omega} \Pi_{\mathscr{V}_{a}} \otimes U_{a}: \quad \mathscr{N} \otimes \mathscr{B}^{\otimes D} \rightarrow \mathscr{N} \otimes \mathscr{B}^{\otimes D}
$$

где $U_{a}$ - произвольные унитарные операторы на $\mathscr{B} \otimes D$. Числа

$$
\mathrm{P}_{U}(a, y)=\mathrm{P}\left(U_{a}|0\rangle, \mathscr{W}_{y}\right) \quad\left(a \in \Omega, y \in \mathbf{B}^{m}\right)
$$

называются условными вероятностями. Говорят, что оператор $U$ измеряет значение функции $F: \Omega \rightarrow \mathbf{B}^{m}$ с вероятностью ошибки не больше $\varepsilon$, если $\mathrm{P}_{U}(a, F(a)) \geqslant 1-\varepsilon$ для всех $a \in \Omega$.

Например, оператор $\bigoplus_{n}[A, D]$ измеряет значение $z_{A}$ с нулевой вероятностью ошибки. Любое квантовое вычисление (см. определение 4.1) можно организовать как измерение по отношению к его входу. Для этого достаточно скопировать входное слово при помоши оператора $\oplus_{n}$ и использовать копию вместо оригинала.

ВАЖный примеР. Пусть $U$ - унитарньй оператор наподпространстве $\mathscr{N} \subseteq \mathscr{B} \otimes n$. Собственные значения этого оператора имеют вид $\lambda(\varphi)=\exp (2 \pi \mathrm{i} \varphi)$, где $\varphi$ - вешественное число по модулю 1 . Соответствуюшие собственные подпространства обозначим через $\mathscr{L}(U, \varphi)$. Пусть оператор $U$ действует на регистре $A$. Добавим еще один квантовый бит и припишем ему номер 1. Тогда оператор

$$
\Xi(U)[A, 1]=S[1] \Lambda(U)[1, A] S[1]
$$

является измеряюшим оператором для квантовой переменной $\varphi$. Если $|\xi\rangle \in \mathscr{L}(U, \varphi)$, то $\Xi(U)|\xi, 0\rangle=|\xi, \eta\rangle$, где

$$
|\eta\rangle=\frac{1}{2}\left(\begin{array}{cc}
1 & 1 \\
1 & -1
\end{array}\right)\left(\begin{array}{cc}
1 & 0 \\
0 & \lambda(\varphi)
\end{array}\right)\left(\begin{array}{cc}
1 & 1 \\
1 & -1
\end{array}\right)\left(\begin{array}{l}
1 \\
0
\end{array}\right)=\left(\begin{array}{c}
\frac{1}{2}(1+\lambda(\varphi)) \\
\frac{1}{2}(1-\lambda(\varphi))
\end{array}\right)
$$


Следовательно, условные вероятности $\mathrm{P}_{\Xi(U)}(\varphi, y)$ равны:

$$
\mathrm{P}_{\Xi(U)}(\varphi, 0)=\frac{1}{2}(1+\cos (2 \pi \varphi)), \quad \mathrm{P}_{\Xi(U)}(\varphi, 1)=\frac{1}{2}(1-\cos (2 \pi \varphi)) .
$$

Общие свойства измеряюших операторов довольно просты. Мы будем рассматривать измеряющие операторы для одной и той же квантовой переменной $z_{\Omega}$, но с различньми дополнительными регистрами $D$.

ПРЕДЛОЖЕНИЕ 5.2. 1. Пусть $U-$ измеряющий оператор. Для любого квантового состояния $|\xi\rangle \in \mathscr{N}$ верна формула полной вероятности

$$
\mathrm{P}\left(U|\xi, 0\rangle, \mathscr{W}_{y}\right)=\sum_{a \in \Omega} \mathrm{P}\left(|\xi\rangle, \mathscr{V}_{a}\right) \mathrm{P}_{U}(a, y)
$$

2. Произведение измеряющих операторов является измеряющим оператором. Измеряющие операторы с непересекающимися дополнительньми регистрами коммутируют.

3. Пусть $U^{\prime}$ и $U^{\prime \prime}$ - измеряющие операторь с непересекающимися дополнительным.ми регистрами $D^{\prime} \supseteq C^{\prime}$ и $D^{\prime \prime} \supseteq C^{\prime \prime}$. Тогда

$$
\mathrm{P}_{U^{\prime} U^{\prime \prime}}\left(a,\left(y^{\prime}, y^{\prime \prime}\right)\right)=\mathrm{P}_{U^{\prime}}\left(a, y^{\prime}\right) \mathrm{P}_{U^{\prime \prime}}\left(a, y^{\prime \prime}\right) \text {. }
$$

ДокаЗАТельство. 1. Ясно, что $U|\xi, 0\rangle=\sum_{a \in \Omega} \Pi_{\mathscr{V}_{a}} U|\xi, 0\rangle$. Следовательно,

$$
\mathrm{P}\left(U|\xi, 0\rangle, \mathscr{W}_{y}\right)=\sum_{a \in \Omega}\left\langle\xi, 0\left|U^{\dagger} \Pi_{\mathscr{V}_{a}} \Pi_{\mathscr{W}_{y}} U\right| \xi, 0\right\rangle=\sum_{a \in \Omega}\left\langle\xi\left|\Pi_{\mathscr{V}_{a}}\right| \xi\right\rangle\left\langle 0\left|U_{a}^{\dagger} \Pi_{\mathscr{W}_{y}} U_{a}\right| 0\right\rangle .
$$

Это дает правую часть формулы (33).

2. Вытекает из определения.

3. Следует из общего свойства квантовой вероятности (11).

Теперь вернемся к примеру, рассмотренному вьше. Предположим, что измеряющий оператор $\Xi(U)$ применяется $s$ раз к одному и тому же регистру $A$ и дополнительным битам $1, \ldots, s$. Построим схему, которая просматривает значения $z_{1}, \ldots, z_{s}$ этих битов и подсчитьвает, сколько из них равно единище. Тем самым мы определяем процедуру, результатом работы которой является число единиц $y$ (а также новое состояние регистра $A)$. Эта процедура описьвается новьм измеряющим оператором $\Xi_{s}(U)$. Поскольку $z_{1}, \ldots, z_{s}$ ведут себя как независимые случайные переменные, величина $y / s$ будет, скорее всего, близка к соответствующей вероятности $\mathrm{P}_{\Xi(U)}(\varphi, 1)$. Точнее говоря, для любой наперед заданной константы $\delta>0$ верна следующая оценка ${ }^{11}$

$$
\operatorname{Prob}\left[\left|y / s-\mathrm{P}_{\Xi(U)}(\varphi, 1)\right|>\delta\right] \leqslant 2 \exp (-c(\delta) s),
$$

где $c(\delta)>0$. Таким образом, мы измеряем величину $\mathrm{P}_{\Xi(U)}(\varphi, 1)=\frac{1}{2}(1-\cos (2 \pi \varphi))$ с точностью $\delta$ и вероятностью ошибки $\leqslant \exp (-c(\delta) s)$. Если заменить $U$ на $\mathrm{i} U$, то $\cos (2 \pi \varphi)$ заменится $-\sin (2 \pi \varphi)$. Следовательно, можно одновременно измерить $\cos (2 \pi \varphi)$ и $\sin (2 \pi \varphi)$; этой информации достаточно, чтобы восстановить $\varphi$. Мы доказали следующее утверждение.

\footnotetext{
${ }^{11}$ Обозначение $\operatorname{Prob}[\cdot]$ было введено в разделе 3.1.
} 
ЛЕмма 5.3. Пусть $\delta>0-$ константа. Для любого $\varepsilon>0$ значение квантовой переменной $\varphi$ может бить измерено с точностью $\delta$ и вероятностью оиибки не больше в при помощи квантовой схемь размера $O(\log (1 / \varepsilon))$ в базисе $\mathscr{Q} \cup\{\Lambda(U)\}$.

$\mathrm{K}$ сожалению, трудно измерить $\varphi$ с произвольной точностью, потому что цена такого измерения (т.е. размер схемы) растет полиномиально по $\delta$. Ситуация резко улучшается, если мы имеем в своем распоряжении операторы $\Lambda\left(U^{k}\right)$ для всех $k$. Точнее говоря, рассмотрим оператор

$$
U^{[0, r]}: \overline{\{0, \ldots, r\}} \otimes \mathscr{N} \rightarrow \overline{\{0, \ldots, r\}} \otimes \mathscr{N}, \quad U^{[0, r]}|k, \xi\rangle=|k\rangle \otimes U^{k}|\xi\rangle,
$$

где $r=2^{l-1}$. Согласно лемме 4.9 , оператор $U^{[0, r]}$ можно преобразовать в $\Lambda\left(U^{2^{j}}\right)$ $(j=0, \ldots, l-1)$. Применим лемму 5.3 к каждому из последних операторов. Таким способом можно локализовать число $2^{j} \varphi(\bmod 1)$ в одном из восьми интервалов $\left[\frac{s-1}{8}, \frac{s+1}{8}\right](s=0, \ldots, 7)$ с вероятностью ошибки $\leqslant \varepsilon / l$. Другими словами, мы находим один из интервалов, в котором лежит неизвестное нам число; для этого требуется произвести $O(\log (l / \varepsilon))$ операций. Используя полученную информацию, можно определить (при помощи полиномиального алгоритма) значение $\varphi$ с точностью $\frac{1}{8} 2^{-(l-1)}=2^{-l-2}$ и вероятностью ошибки $\leqslant \varepsilon$. Мы получили следующий результат.

ЛЕМма 5.4. Пусть $l$ - положительное иелое число; $r=2^{l-1}$. Для любо$2 о \varepsilon>0$, значение переменной $\varphi$ может бить измерено $c$ точностью $2^{-l-2}$ и вероятностью ошибки не больше в при помощи квантовой схемы размера $O(l \log (l / \varepsilon))+\operatorname{poly}(l)$ в базисе $\mathscr{Q} \cup\left\{U^{[0, r]}\right\}$. (Элемент $U^{[0, r]}$ используется не более $O(l \log (l / \varepsilon))$ раз. $)$

Теперь рассмотрим важный частный случай: $U=\widehat{g}$, где $g$ - перестановка на множестве $N \subseteq \mathbf{B}^{n}$. Каждому циклу этой перестановки отвечают собственные значения вида $\exp \left(2 \pi \mathrm{i} \frac{p}{q}\right)$, где $q$ - длина цикла. Таким образом, возможные значения $\varphi$ - это рациональные числа со знаменателями $\leqslant 2^{n}$. Наименьшее расстояние между такими числами равно $\left(2^{n}\left(2^{n}-1\right)\right)^{-1}$. Следовательно, чтобы измерить $\varphi$ точно, достаточно измерить его с точностью $2^{-2 n-1}$. Более того, переход от приближенного значения к точному осуществляется за полиномиальное время; для этого нужно воспользоваться алгоритмом цепных дробей. Мы доказали следующую теорему.

Теорема 5.5. Пусть $g$ - перестановка на множестве $N \subseteq \mathbf{B}^{n}$. Собственное значение оператора $U=\widehat{g}$ может быть измерено с вероятностью ошибки не больше в при помощи квантовой схемь размера poly $(n)+O(n) \log (1 / \varepsilon)$ в базисе $\mathscr{Q} \cup\left\{U^{\left[0,2^{2 n}\right]}\right\}$. (Элемент $U^{\left[0,2^{2 n}\right]}$ используется не более $O(n \log (n / \varepsilon))$ раз.) Эта схема строится алгоритмически за время $\operatorname{poly}(n+\log (1 / \varepsilon))$.

\section{6. Полиномиальный квантовый алгоритм для задачи о стабилизаторе}

Пусть $(k, n, a, F)$ - представитель абелевой задачи о стабилизаторе, $\operatorname{St}_{F}(a)=$ $\left\{g \in \mathbf{Z}^{k}: F(g, a)=a\right\}$ - его решение. Для упрощения обозначений мы будем писать $g(a)$ вместо $F(g, a)$. Очевидно, что $g(a)$ зависит только от образа $g$ в фактор-группе $E=\mathbf{Z}^{k} / \operatorname{St}_{F}(a)$. Этот образ, обозначаемьй как $\theta(g)$, может рассматриваться как перестановка на множестве $N=\left\{g(a): g \in \mathbf{Z}^{k}\right\} \subseteq \mathbf{B}^{n}$ (орбите элемента $a$ ). Ключевая идея состоит в том, чтобы измерить собственные значения операторов 
$\widehat{\theta\left(g_{j}\right)}, j=1, \ldots, k$, где $g_{1}, \ldots, g_{k}$ - образующие группы $\mathbf{Z}^{k}$. (Разумеется, эти измерения - только часть более сложной процедуры.)

Возможные комбинации собственных значений описываются группой характеров на $E$,

$$
\mathrm{H}=\operatorname{Hom}(E, \mathbf{Q} / \mathbf{Z}) \subseteq \operatorname{Hom}\left(\mathbf{Z}^{k}, \mathbf{Q} / \mathbf{Z}\right)=(\mathbf{Q} / \mathbf{Z})^{k} .
$$

Каждый элемент $h$ группы $H$ можно представить $k$ рациональными числами $(\bmod 1) \varphi_{1}, \ldots, \varphi_{k} \in \mathbf{Q} / \mathbf{Z}$ с обшим знаменателем $q=|E|=|H| \leqslant 2^{n}$. А именно, $\varphi_{j}=\left(h, \theta\left(g_{j}\right)\right)$, где $(\cdot, \cdot): H \times E \rightarrow \mathbf{Q} / \mathbf{Z}$ - естественное спаривание. Все операторы $\widehat{\theta(g)}: g \in \mathbf{Z}^{k}$ имеют одинаковый набор собственных векторов

$$
\left|\psi_{h}\right\rangle=\frac{1}{\sqrt{q}} \sum_{u \in E} \exp (-2 \pi \mathrm{i}(h, u))|u(a)\rangle \quad(h \in H) .
$$

Соответствуюшие собственные значения равны $\lambda_{h}(g)=\exp (2 \pi \mathrm{i}(h, \theta(g)))$.

Теорема 5.5 позволяет измерить значение $\varphi_{j}=\left(h, \theta\left(g_{j}\right)\right)$ для каждого $j$ (используя операторы $\left.\widehat{\theta\left(g_{j}\right)}\right)$. Эти измерения не мешают друг другу, поскольку они относятся к одной и той же системе взаимно ортогональных подпространств (см. предложение 5.2). Следовательно, можно измерить все числа $\varphi_{j}=\left(h_{j}, \theta\left(g_{j}\right)\right)$ одновременно. Таким образом, квантовая переменная $h=\left(\varphi_{1}, \ldots, \varphi_{k}\right) \in(\mathbf{Q} / \mathbf{Z})^{k}$ может быть измерена с вероятностью ошибки $\leqslant k \varepsilon$ при помоши схемы размера $k(\operatorname{poly}(n)+O(n) \log (1 / \varepsilon))$ в базисе $\mathscr{Q} \cup\{\widehat{G \mid O(n)}\}$. (Отображение $G$ определено по формуле (6).)

Теперь вернемся к исходной задаче. Ясно, что вычисление $\operatorname{St}_{F}(a)$ полиномиально эквивалентно вычислению $H$. (Найти $H$ - значит найти полиномиальное подмножество в $(\mathbf{Q} / \mathbf{Z})^{k}$, которое порождает $H$.) Группа $H$ порождается достаточным количеством случайных элементов (см. ниже). Сейчас мы опишем метод, позволяюший породить один случайньй элемент.

Здесь используется новый трюк (сравните с [13]). Приготовим базисное состояние $|a\rangle$ и измерим $h$. Очевидно, что

$$
|a\rangle=\frac{1}{\sqrt{q}} \sum_{h \in H}\left|\psi_{h}\right\rangle
$$

Согласно формуле полной вероятности (33), вероятность получить результат $y$ равна

$$
\operatorname{Prob}[h=y]=q^{-1} \sum_{h \in H} \mathrm{P}_{U}(h, y), \quad \text { где } \mathrm{P}_{U}(h, h) \geqslant 1-k \varepsilon, \sum_{y: y \neq h} \mathrm{P}_{U}(h, y) \leqslant k \varepsilon .
$$

(Здесь $U$ обозначает измеряющий оператор.) Следовательно,

$$
q^{-1}|L|(1-k \varepsilon) \leqslant \operatorname{Prob}[h \in L] \leqslant q^{-1}|L|+k \varepsilon \text { для любого } L \subseteq H .
$$

Таким способом мы порождаем случайньй элемент $H$ с почти равномерным распределением.

Пусть $h_{1}, \ldots, h_{l} \in(\mathbf{Q} / \mathbf{Z})^{k}$ - независимые случайные элементы, полученные этим способом. Мы собираемся показать, что они почти наверняка порождают $H$, если выбрать подходящее $l$. 
Все элементы $h_{1}, \ldots, h_{l}$ принадлежат $H$ с вероятностью $\geqslant 1-k l \varepsilon$. Допустим, что они принадлежат $H$, но не порождают $H$. Тогда $h_{1}, \ldots, h_{l} \in L$, где $L$ - максимальная собственная подгруппа $H$. Для каждого $L$ вероятность этого события не превьшает $(\operatorname{Prob}[h \in L])^{l} \leqslant\left(\frac{1}{2}+k \varepsilon\right)^{l}$ (в силу неравенства $\left.|L| \leqslant|H| / 2\right)$. Максимальные собственные подгрупшы $H$ находятся во взаимно-однозначном соответствии с минимальными ненулевьми подгруппами $E$. Число таких подгруп меншше, чем $|E| \leqslant 2^{n}$. Следовательно, суммарная вероятность того, что $h_{1}, \ldots, h_{l}$ не порождают $H$, меньше, чем $k l \varepsilon+2^{n-l}(1+2 k \varepsilon)^{l}$. (Первое слагаемое соответствует случаю $\left\{h_{1}, \ldots, h_{l}\right\} \nsubseteq H, \mathrm{a}$ второе учитьвает все возможные подгруппы $L$.) Положим $l=n+4, \varepsilon=(6 k l)^{-1}$. Тогда случайные элементы $h_{1}, \ldots, h_{l} \in(\mathbf{Q} / \mathbf{Z})^{k}$ порождают $H$ с вероятностью $\geqslant \frac{2}{3}$.

Итак, все вычисление в целом происходит следующим образом. Мы берем $l=n+4$ регистров и приготавливаем в каждом из них начальное состояние $|a\rangle$. Затем мы производим $O(k n \log (k n))$ элементарных измерений $\Xi\left({\widehat{g_{j}}}^{2^{s}}\right)(1 \leqslant s \leqslant 2 n)$ с каждым регистром. Результаты этих измерений обрабатьваются классически; таким способом мы находим $h_{1}, \ldots, h_{l}$ и, в конечном счете, канонический базис стабилизатора (с вероятностью ошибки $\left.\leqslant \frac{1}{3}\right)$. K оракулу $F$ приходится обрашаться $O\left(k n^{2} \log (k n)\right)$ раз; каждый вопрос к оракулу имеет длину $O(n)$. Подчеркнем, что описанная процедура является однородной, т.е. соответствующая квантовая схема строится алгоритмически за время $\operatorname{poly}(k+n)$.

\section{7. Вычисления с возмущениями: выбор модели}

Мы переходим к новой теме - борьбе с возмущениями. Проблема состоит в следующем. Имеется произвольная последовательность операций, представляющая некоторый унитарньй оператор или вычисляющая некоторую булеву функцию. Нужно реализовать тот же оператор или ту же функцию при помоши другой последовательности операций, которая была бы устойчива к возмущениям. В буквальном смысле это невозможно. Корректная формулировка задачи (см. раздел 10.1) основана на понятии неоднозначного кода. Цель этого раздела - сформулировать определенные (достаточно реалистические) предположения о характере возмущений, выбрав подходящую для этого вычислительную модель.

В обшем случае возмушение описывается унитарным оператором, действующим на полном пространстве состояний кубитов и окружаюшей среды. Предполагая, что окружение обновляется на каждом шаге, мы можем исключить его из рассмотрения и перейти на язык преобразований операторов плотности (см. раздел 3.2). (Это предположение означает статистическую независимость ошибок на разных шагах.) Удобно с самого начала считать, что квантовый компьютер работает со смешанньми состояниями, и даже невозмущенные элементарные преобразования не обязательно имеют вид $U \cdot U^{\dagger}$. Такие вычисления назьваются смешанным.м.

Как было отмечено во введении, возмущения действуют на квантовые биты даже тогда, когда никаких операций не производится. Поэтому мы вынуждены рассматривать процесс вычисления в реальном времени. Точнее говоря, нам придется перейти от последовательных вычислений к параллельным.

На каждом шаге параллельного смешанного вычисления все биты разбиваются на небольшие группы (регистры) $A_{1}, \ldots, A_{s}$ по 1-3 бита в каждом. К каждому регистру $A_{j}$ применяется какое-нибудь допустимое преобразование $T_{j}$ из заданного базиса $\mathscr{A}$. (Возможно, почти все регистры $A_{j}$ состоят из одного бита, а соответствуюшие преоб- 
разования $T_{j}$ - тождественные.) Результат преобразования $T_{j}$ записьвается в новьй регистр $A_{j}^{\prime}$, длина которого может отличаться от длины регистра $A_{j}$. Эта операция обозначается символом $T_{j}\left[A_{j}^{\prime} ; A_{j}\right]$. Некоторые из полученных битов могут игнорироваться, другие используются на следуюшем шаге. Последовательность таких шагов называется слоистой схемой. Вот формальное определение.

ОПРЕДЕЛЕНИЕ 7.1. Слоистая схема глубинь $d$ есть совокупность следующих объектов:

- набор конечных множеств $\Delta_{0}, \ldots, \Delta_{d}$, назьваемых слоями; нулевой слой $\Delta_{0}$ называется входом схемы, а последний слой $\Delta_{d}-$ выходом;

- разбиения каждого из множеств $\Delta_{i-1}(i=1, \ldots, d)$ на упорядоченные подмножества (регистры) $A_{i 1}, \ldots, A_{i s_{i}}$, а также разбиения некоторых множеств $\Delta_{i}^{\prime} \supseteq \Delta_{i}$ на регистры $A_{i 1}^{\prime}, \ldots, A_{i s_{i}}^{\prime}$;

- набор допустимых преобразований $T_{i j} \in \mathbf{T}\left(\mathscr{B}^{\otimes n_{i j}, \mathscr{B}} \otimes m_{i j}\right) \quad\left(n_{i j}=\left|A_{i j}\right|\right.$, $\left.m_{i j}=\left|A_{i j}^{\prime}\right|\right)$ из некоторого базиса $\mathscr{A} \ni I_{\mathscr{B}}$; преобразования $T_{i j}$ (или их номера $(i, j))$ назьваются элементами; полное число элементов назьвается размером схемы (напомним, что $I_{\mathscr{B}}$ обозначает тождественное преобразование одного кубита).

Такая схема $\Phi$ реализует преобразование $\operatorname{Trans}(\Phi)=T_{d} \cdots T_{1}$, где

$T_{i} \rho=\operatorname{Tr}_{\Delta_{i}^{\prime} \backslash \Delta_{i}}\left(T_{i 1}\left[A_{i 1}^{\prime} ; A_{i 1}\right] \otimes \cdots \otimes T_{i s_{i}}\left[A_{i s_{i}}^{\prime} ; A_{i s_{i}}\right] \rho\right) \quad$ для любого $\rho \in \mathbf{L}\left(\mathscr{B}^{\otimes \Delta_{i-1}}\right)$.

При наличии возмушений каждое из преобразований $T_{i j}$ заменяется на произвольное допустимое преобразование $\widetilde{T}_{i j}$ такое, что $\left\|\widetilde{T}_{i j}-T_{i j}\right\|_{\diamond} \leqslant \delta$. В результате получается некоторое множество $\operatorname{Trans}(\Phi, \delta)$ преобразований, реализуемых схемой $\Phi$ с неточньми элементами. Константа точности $\delta$ будет предполагаться достаточно малой, но не зависящей от конкретной слоистой схемы.

В качестве базисных элементов для построения слоистых схем естественно взять все преобразования вида $U \cdot U^{\dagger}: U \in \mathscr{Q}$, а также преобразование, создающее состояние $|0\rangle$. (Напомним, что $\mathscr{Q}$ обозначает стандартный базис квантовых вычислений.) Однако мы будем использовать более обширньй базис, включаюший в себя как квантовые, так и классические элементы. Это предполагает небольшую модификацию описанной выше модели.

Все биты в схеме делятся на квантовые и классические. В качестве элементов разрешается использовать допустимые комбинированные преобразования вида

$$
T \in \mathbf{T}\left(\mathscr{B}^{\otimes n} \times \mathbf{B}^{k}, \mathscr{B}^{\otimes m} \times \mathbf{B}^{l}\right) \subseteq \mathbf{T}\left(\mathscr{B}^{\otimes(n+k)}, \mathscr{B}^{\otimes(m+l)}\right) .
$$

(Преобразование $T$ можно применить к $n$ квантовым и $k$ классическим битам.) Такие комбинированные вычисления являются частным случаем смешанных. Естественно, однако, предположить, что возмушение оставляет классические биты в классическом состоянии. Например, если к классическому регистру $A$ применяется булева функция $f$, то возмущенное преобразование описывается матрицей переходов $t$ (см. (15)). Условие точности в этом случае приобретает вид $\forall a \in \mathbf{B}^{A}\left(1-t_{f(a), a} \leqslant \delta / 2\right)$. Величина $1-t_{f(a), a}$ имеет смысл вероятности ошибки.

Базис для комбинированных вычислений можно выбрать следуюшим образом. Возьмем какой-нибудь классический базис $\mathscr{A}_{c}$ (состоящий из булевых функций) и 
чисто квантовьй базис $\mathscr{A}_{q}$ (состоящий из унитарных операторов). В соответствующий комбинированный базис $\mathscr{A}$ будут входить следующие элементы:

- преобразования $f \in \mathscr{A}_{c} \cup\{\nabla\}$ с классическими битами (напомним, что $\nabla$ - это операция копирования одного бита);

- преобразования $U \cdot U^{\dagger}: U \in \mathscr{A}_{q}$ с квантовыми битами;

- преобразование, создающее квантовое состояние $|0\rangle$;

- преобразование $Q_{\mathbf{B}}^{*}$, преврашающее квантовьй бит в классический;

- комбинированные преобразования $\Lambda_{c}(U): U \in \mathscr{A}_{q}$ с одним классическим ynравляющим битом и несколькими квантовыми битами

$$
\begin{gathered}
\Lambda_{c}(U)(a, \rho)= \begin{cases}(0, \rho), & \text { если } a=0, \\
\left(1, U \rho U^{\dagger}\right), & \text { если } a=1,\end{cases} \\
\Lambda_{c}(U): \mathbf{B} \times \mathbf{L}\left(\mathscr{B}^{\otimes n}\right) \rightarrow \mathbf{B} \times \mathbf{L}\left(\mathscr{B}^{\otimes n}\right) .
\end{gathered}
$$

В дальнейшем мы будем, в основном, работать с полным базисом $\mathscr{Q} C$, которьй задается следуюшим выбором $\mathscr{A}_{q}$ и $\mathscr{A}_{c}$ :

$$
\mathscr{A}_{c}=\mathscr{C}=\left\{I_{\mathbf{B}}, 0, \neg, \wedge\right\}, \quad \mathscr{A}_{q}=\mathscr{Q}=\mathscr{S} \cup\left\{\widehat{\wedge}_{\oplus}\right\}, \quad \text { где } \mathscr{S}=\left\{1_{\mathscr{B}}, S, K, \widehat{\oplus}\right\} .
$$

(Операторы $S$ и $K$ определяются формулой (24).) Квантовый базис $\mathscr{S}$ назьвается симплектическим, так как он порождает расширенную симплектическую группу $\operatorname{ESp}_{2}(n)$ (см. раздел 9.1). Соответствующий комбинированньй базис $\mathscr{S} C$ называется симплекто-классическим. Этот базис неполон, т.е. не позволяет реализовать произвольное допустимое преобразование с произвольной точностью. Однако в нем можно выполнять такие полезные процедуры, как кодирование, декодирование и исправление ошибок. По этой причине проблему возмушений для симплекто-классического базиса можно рассмотривать отдельно; она решается несколько проще, чем в случае полного базиса.

\section{8. Квантовые коды (определения и общие свойства)}

8.1. Основные понятия и идеи. Рассмотрим упрощенную задачу о борьбе с возмушениями. Допустим, что возмушение действует только на одном шаге. До и после этого шага разрешается выполнить произвольные преобразования - кодирование и декодирование. Такая ситуация имеет место при передаче квантового состояния между двумя идеальными устройствами по каналу с шумом. Требуется предать квантовое состояние $m$-битного регистра с достаточно малой вероятностью ошибки, используя $n$-битньй канал связи.

Напомним классический вариант этой задачи. Классический канал описьвается матрицей вероятностей переходов $\left(t_{i j}: x \in N, y \in N^{\prime}\right)$, где $N$ и $N^{\prime}$ - множества входных и выходных сигналов. Имеется и другая, более грубая, модель: все переходы $x \mapsto y$ делятся на "вероятные" и "маловероятные" так, чтобы суммарная (для данного $x$ ) вероятность "маловероятных" переходов не превьшала заданное число $\varepsilon$. В этом случае мы имеем дело лишш с множеством "вероятных" переходов $E \subseteq N \times N^{\prime}$. Рассмотрим, например, $n$-битный классический канал с независимыми ошибками: $N=$ $N^{\prime}=\mathbf{B}^{n}, t_{x y}=p^{d(x, y)}(1-p)^{n-d(x, y)}$, где $p$ - параметр (вероятнось ошибки в одном 
бите), a $d(\cdot, \cdot)$ - расстояние Хэмминга. В этом случае "вероятньми" являются переходы с $d(x, y) \geqslant k=\mathrm{const} ;$ множество таких переходов обозначается через $E(n, k)$.

Классический код типа $(N, M)$ - это произвольное вложение $h: M \rightarrow N$. (Элементы множества $M$ имеют смысл сообщений, которые кодируются, а затем передаются по каналу.) Восстановление исходного сообшения возможно, если любые два различных элемента $x_{1}, x_{2} \in L=\operatorname{Im} h$ не "склеиваются", т.е. не существует такого $y$, что $\left(x_{1}, y\right) \in E$ и $\left(x_{2}, y\right) \in E$. В случае $E=E(n, k)$ говорят, что код исправляет $k$ ошибок. Задача состоит в поиске кодов, обладаюших этим свойством. (При этом обычно требуется, чтобы мошность кода $|L|$ была достаточно большой; существуют и другие критерии "качества" кодов.)

Квантовый канал описьвается допустимьм преобразованием $\widetilde{T} \in \mathbf{T}\left(\mathscr{N}, \mathscr{N}^{\prime}\right)$. Более грубая модель квантового канала задается линейным пространством ошибок $\mathscr{E} \subseteq \mathbf{L}\left(\mathscr{N}, \mathscr{N}^{\prime}\right)$, которое имеет тот же смысл, что и множество "вероятных" переходов в классическом случае. Конкретизируем эти модели и укажем связь между ними.

Мы будем рассматривать следуюший класс каналов: $\mathscr{N}=\mathscr{N}^{\prime}=\mathscr{B}^{n}, \widetilde{T}=$ $\widetilde{T}_{1} \otimes \cdots \otimes \widetilde{T}_{n}$, где $\widetilde{T}_{j}-$ допустимые преобразования с одним кубитом такие, что $\left\|\widetilde{T}_{j}-I_{\mathscr{B}}\right\|_{\diamond} \leqslant \delta$. (Здесь $\delta$ - некоторая константа.) Для каждого подмножества $A \subseteq$ $\Delta=\{1, \ldots, n\}$ определим пространство ошибок с носителем $A$, т.е. пространство линейных операторов, действуюших только на кубиты из $A$

$$
\mathscr{E}(A)=\left\{X[A]: X \in \mathbf{L}\left(\mathscr{B}^{\otimes|A|}\right)\right\} \subseteq \mathbf{L}\left(\mathscr{B}^{\otimes \Delta}\right) .
$$

Пространству операторов $\mathscr{E}(A)$ соответствует пространство преобразований $\Re(A)=$ $\mathscr{E}(A) \cdot \mathscr{E}(A)^{*}$. Положим

$$
\mathscr{E}(n, k)=\sum_{A \subseteq \Delta:|A| \leqslant k} \mathscr{E}(A), \quad \Re(n, k)=\sum_{A \subseteq \Delta:|A| \leqslant k} \Re(A) .
$$

(Очевидно, что $\Re(n, k) \subseteq \mathscr{E}(n, k) \cdot \mathscr{E}(n, k)^{*}$.) В этой ситуации имеет место следующее

ПрЕДЛОЖЕНИЕ 8.1. Пусть $\widetilde{T}=\widetilde{T}_{1} \otimes \cdots \otimes \widetilde{T}_{n}$, где $\widetilde{T}_{j}-$ допустимое преобразование с одним кубитом такое, что $\left\|\widetilde{T}_{j}-I_{\mathscr{B}}\right\|_{\diamond} \leqslant \delta($ для каждого $j)$. Тогда существует такое преобразование $T \in \Re(n, k)$, что

$$
\|T-\widetilde{T}\|_{\diamond} \leqslant \sum_{l>k}\left(\begin{array}{l}
n \\
l
\end{array}\right) \delta^{l} .
$$

ДокАЗАТЕЛЬСТвО. Раскройте скобки в выражении $\widetilde{T}=\bigotimes_{j=1}^{n}\left(I_{\mathscr{B}}+\left(\widetilde{T}_{j}-I_{\mathscr{B}}\right)\right)$.

(Однозначным) квантовыц кодом типа $(\mathscr{N}, \mathscr{M})$ назьвается любое унитарное вложение $V: \mathscr{M} \rightarrow \mathscr{N}$, заданное с точностью до фазового множителя. (Неоднозначные коды будут определены в разделе 8.3.) Пространство $\mathscr{N}$ назьвается кодовым., а пространство $\mathscr{M}$ - информационныц. Если $\mathscr{N}=\mathscr{B}^{\otimes n}$ и $\mathscr{M}=\mathscr{B}^{\otimes m}$, то $V$ называется кодом типа $(n, m)$.

Исправление ошибок описьвается некоторьм допустимым преобразованием $P \in$ $\mathbf{T}\left(\mathscr{N}^{\prime}, \mathscr{M}\right)$, см. определение 8.2 ниже. Свойство кода исправлять ошибки зависит не от самого вложения $V$, а от информационного подпространства $\mathscr{L}=V \mathscr{M} \subseteq \mathscr{N}$. Квантовый код $V: \mathscr{M} \rightarrow \mathscr{N}$ исправляет ошибки из пространства $\mathscr{E} \subseteq \mathbf{L}\left(\mathscr{N}, \mathscr{N}^{\prime}\right)$, 
если ортогональные векторы "не склеиваются". Точнее говоря, для любой пары ортогональных векторов $|\xi\rangle,|\eta\rangle \in \mathscr{L}$ и пары операторов $X, Y \subseteq \mathscr{E}$ векторы $X|\xi\rangle$ и $Y|\eta\rangle$ также ортогональны (см. теорему 8.3 ниже).

В классическом случае существует очень простой код $\operatorname{REP}(n)$, исправляющий $\left\lfloor\frac{n-1}{2}\right\rfloor$ ошибок (см. введение). Почему же этот пример не обобщается на квантовьй случай? Можно, конечно, определить вложение $\mathscr{B} \rightarrow \mathscr{B} \otimes n$ по формулам $|0\rangle \mapsto$ $|0, \ldots, 0\rangle,|1\rangle \mapsto|1, \ldots, 1\rangle$. Однако такой квантовьй код не зашищает даже от одной ошибки, точнее говоря, он не исправляет ошибок из пространства $\mathscr{E}(n, 1)$. В самом деле, воспользуемся упомянутым выше критерием. Рассмотрим следуюшую пару ортогональных векторов из информационного подпространства:

$$
|\xi\rangle=2^{-1 / 2}(|0, \ldots, 0\rangle+|1, \ldots, 1\rangle), \quad|\eta\rangle=2^{-1 / 2}(|0, \ldots, 0\rangle-|1, \ldots, 1\rangle) .
$$

Пусть $X=1, Y=\sigma_{z}[j]$ (j- любое). Тогда $X|\xi\rangle=Y|\eta\rangle=|\xi\rangle-$ критерий не выполнен. Заметим, что ошибка вида $\sigma_{z}[j]$ (так называемая фазовая ошибка) не влияет на базисные состояния и поэтому не имеет классического аналога. Напротив, классические ошибки вида $\sigma_{x}[j]$ исправляются рассматриваемым кодом. Таким образом, трудность построения квантовых кодов состоит в том, что нужно исправлять классические и фазовые ошибки одновременно. Простейший известный пример квантового кода, исправляюшего одну ошибку (пример 9.3), достаточно нетривиален.

Среди классических кодов наиболее хорошо изучены линейные коды над полем $\mathbf{F}_{2}$. Множество кодовых слов $L \subseteq \mathbf{B}^{n}=\mathbf{F}_{2}^{n}$ линейного кода характеризуется нулевым значением нескольких контрольных сумм, т.е. некоторых линейных функций вида $\mathbf{F}_{2}^{n} \rightarrow \mathbf{F}_{2}$. Квантовым аналогом функции является оператор, а аналогом линейной функции - оператор вида $\pm \sigma_{\gamma_{1}} \otimes \cdots \otimes \sigma_{\gamma_{n}}\left(\gamma_{j} \in\{0, x, y, z\}\right)$, где $\sigma_{0}=1_{\mathscr{B}}$. Пусть задано несколько операторов такого вида, назьваемых проверочными операторами. Информационное подпространство $\mathscr{L} \subseteq \mathscr{B}^{\otimes n}$ определяется как множество векторов, инвариантных относительно действия этих операторов. Это определение имеет смысл только в том случае, если проверочные операторы коммутируют друг с другом. Условие коммутации можно записать при помоши канонической симплектической формы над $\mathbf{F}_{2}$, поэтому коды такого типа назьвается симллектическими. Данная конструкшия будет подробно описана в разделе 9. Ближайшие два раздела (8.2 и 8.3) посвяшены изучению обших свойств квантовых кодов.

8.2. Однозначные коды. Пусть $V: \mathscr{M} \rightarrow \mathscr{N}$ - квантовый код, $\mathscr{L}=V \mathscr{M} \subseteq \mathscr{N}$ информационное подпространство.

ОПРЕДЕЛЕНИЕ 8.2. Пусть $\Re \subseteq \mathbf{T}\left(\mathscr{N}, \mathscr{N}^{\prime}\right)$. Говорят, что код $V$ исправляет ошибки из $\Re$, если сушествует допустимое преобразование $P \in \mathbf{T}\left(\mathscr{N}^{\prime}, \mathscr{N}\right)$ и функция $c: \Re \rightarrow \mathbf{C}$ такие, что

$$
P T \rho=c(T) \rho \text { для любых } T \in \Re, \quad \rho \in \mathbf{L}(\mathscr{L}) .
$$

Преобразование $P$ назьвается исправляющим. В случае $\Re=\mathscr{E} \cdot \mathscr{E}^{*}\left(\right.$ где $\mathscr{E} \subseteq \mathbf{L}\left(\mathscr{N}, \mathscr{N}^{\prime}\right)$ - некоторое линейное подпространство) говорят, что код $V$ исправляет ошибки из $\mathscr{E}$, а $\mathscr{E}$ назьвают пространством ошибок. Если $\mathscr{E}=\mathscr{E}(n, k)$, то говорят, что код $V$ исправляет $k$ оиибок.

Заметим, что если преобразование $T$ является допустимьм, то $c(T)=1$. В самом деле, $c(T) 1_{\mathscr{M}}=\left(T\left(V \cdot V^{\dagger}\right)\right)^{*} 1_{\mathscr{N}^{\prime}}$. 
Посмотрим, что дает это определение для канала, описьваемого произвольным допустимьм преобразованием $\widetilde{T} \in \mathbf{T}\left(\mathscr{N}, \mathscr{N}^{\prime}\right)$. Предположим, что сушествует $T \in \Re$ такое, что $\|T-\widetilde{T}\|_{\diamond} \leqslant \varepsilon$ (ср. с предложением 8.1). Тогда $|c(T)-1| \leqslant \varepsilon$, следовательно,

$$
\left\|P \widetilde{T}\left(V \cdot V^{\dagger}\right)-I_{\mathscr{M}}\right\|_{\diamond} \leqslant 2 \varepsilon .
$$

Таким образом, квантовые коды можно использовать для приближенного восстановления квантового состояния после действия возмушения.

Обозначим через $\mathbf{L}_{0}(\mathscr{M})$ центр алгебры $\mathbf{L}(\mathscr{M})$, т.е. множество операторов вида $c 1_{\mathscr{M}}: c \in \mathbf{C}$.

Теорема 8.3. Пусть $\Re \subseteq \mathrm{T}\left(\mathscr{N}, \mathscr{N}^{\prime}\right)$ - некоторое множество вполне положительных преобразований. Определим минимальное пространство операторов $\mathscr{E} \subseteq \mathbf{L}\left(\mathscr{N}, \mathscr{N}^{\prime}\right)$ такое, что $\Re \subseteq \mathscr{E} \cdot \mathscr{E}^{*}$. Следующие условия эквивалентны:

1) код $V$ исправляет ошибки из $\mathscr{E}$;

2) код $V$ исправляет ошибки из

3) для любой пары ортогональных векторов $|\xi\rangle,|\eta\rangle \in \mathscr{L}$ и парь операторов $X, Y \subseteq \mathscr{E}$ векторы $X|\xi\rangle$ и $Y|\eta\rangle$ ортогональнь;

4) $V^{\dagger} Y^{\dagger} X V \in \mathbf{L}_{0}(\mathscr{M})$ для любых $X, Y \in \mathscr{E}$.

ДОКАЗАТЕЛЬСТвО. 1) $\Rightarrow 2)$. Очевидно.

$2) \Rightarrow 3)$. Каждое преобразование $T \in \Re$ можно представить в виде $\sum_{j} X_{T j} \cdot X_{T j}^{\dagger}$. Операторы $X_{T j}$ порождают пространство $\mathscr{E}$. Достаточно показать, что векторы $X_{T j}|\xi\rangle$ и $X_{R k}|\eta\rangle$ ортогональны для любых $T, j, R, k$.

Пусть $\mathscr{G}_{1}, \mathscr{G}_{2} \subseteq \mathscr{L}$ - взаимно дополнительные ортогональные подпространства такие, что $|\xi\rangle \in \mathscr{G}_{1},|\eta\rangle \in \mathscr{G}_{2}$. Обозначим через $\Pi_{1}$ и $\Pi_{2}$ соответствуюшие проекторы. Операторы $H_{1}=P^{*} \Pi_{1}$ и $H_{2}=P^{*} \Pi_{2}$ эрмитовы и положительны, причем $H_{1}+H_{2}=1_{\mathscr{N}}$. Очевидно, что

$$
\sum_{j}\left\langle X_{T j} \xi\left|H_{2}\right| X_{T j} \xi\right\rangle=\operatorname{Tr}\left(H_{2}(T|\xi\rangle\langle\xi|)\right)=\operatorname{Tr}\left(\Pi_{2}(P T|\xi\rangle\langle\xi|)\right)=c(T)\left\langle\xi\left|\Pi_{2}\right| \xi\right\rangle=0 .
$$

Следовательно, каждое слагаемое в левой части равно нулю. Аналогично, если $|\eta\rangle \in \mathscr{G}_{2}$, то $\left\langle X_{R k} \eta\left|H_{1}\right| X_{R k} \eta\right\rangle=0$. Отсюда вытекает, что векторы $\left|X_{T j} \xi\right\rangle$ и $\left|X_{R k} \eta\right\rangle$ ортогональны.

$3) \Rightarrow 4)$. Пусть $Z=V^{\dagger} Y^{\dagger} X V \in \mathbf{L}(\mathscr{M})$. Если $\langle\xi \mid \eta\rangle=0$, то $\langle\xi|Z| \eta\rangle=0$. Следовательно, оператор $Z$ коммутирует с проекторами. Однако линейная оболочка всех проекторов совпадает с $\mathbf{L}(\mathscr{M})$, поэтому $Z$ коммутирует с любыми операторами.

$4) \Rightarrow 1)$. Пусть $\mathscr{F}=\{X V: X \in \mathscr{E}\}$. Условие 4) позволяет определить на пространстве $\mathscr{F}$ эрмитово скалярное произведение $\langle\cdot \mid \cdot\rangle$ такое, что $B^{\dagger} A=$ $\langle B, A\rangle 1_{\mathscr{M}}$ (для любых $A, B \in \mathscr{F}$ ). Естественным образом определяетя линейньй оператор $U: \mathscr{M} \otimes \mathscr{F} \rightarrow \mathscr{N}^{\prime}$ такой, что $U(|\xi\rangle \otimes A)=A|\xi\rangle$ (для любых $|\xi\rangle \in \mathscr{M}$ и $A \in \mathscr{F})$. Очевидно, что оператор $U$ сохраняет скалярное произведение, т.е. является унитарным вложением.

Пусть $\mathscr{K}$ - ортогональное дополнение к подпространству $U(\mathscr{M} \otimes \mathscr{F}) \subseteq \mathscr{N}^{\prime}$, а $W: \mathscr{K} \rightarrow \mathscr{N}^{\prime}$ - естественное вложение. Выберем произвольное допустимое преобразование $R \in \mathbf{T}(\mathscr{K}, \mathscr{M})$ и положим

$$
P=\left(V \cdot V^{\dagger}\right)\left(\operatorname{Tr}_{\mathscr{F}}\left(U^{\dagger} \cdot U\right)+R\left(W^{\dagger} \cdot W\right)\right) .
$$


Функция $c: \mathscr{E} \cdot \mathscr{E}^{*} \rightarrow \mathbf{C}$ определяется по формуле $c\left(X \cdot Y^{\dagger}\right)=\langle Y V, X V\rangle$. Легко проверить, что преобразование $P$ и функция $c$ обладают требуемыми свойствами.

8.3. Неоднозначные коды. При моделировании схем с точными элементами схемами с неточньми элементами используются неоднозначные коды. Дело в том, что исправляющее преобразование для обычного кода нельзя реализовать схемой из неточных элементов. (Неточность на последнем шаге всегда приводит к неоднозначности.) Простейший пример неоднозначного классического кода - это код $\operatorname{REP}(n, l)$ типа $(n, 1)$. Ноль кодируется любым словом длины $n$, содержашим не более $l$ единиц; единица кодируется любым словом длины $n$, содержащим не более $l$ нулей. (Это определение корректно при $2 l<n$.) Код $\operatorname{REP}(n, l)$ исправляет $\left\lfloor\frac{n-1}{2}\right\rfloor-l$ ошибок. Для задачи о передаче информации (или квантового состояния) неоднозначные коды не дают никакого вьигрьша по сравнению с однозначньмми.

Пусть $N$ и $M$ - конечные множества. Неоднозначныл классическим кодом типа $(N, M)$ назьвается пара $(L, f)$, где $L \subseteq N$, а $f: L \rightarrow M$ - сюръективное отображение. Состояние $a \in M$ кодируется любым состоянием $b \in f^{-1}(a)$.

Пусть $\mathscr{N}$ и $\mathscr{M}$ - унитарные пространства. Неоднозначныцм квантовыц кодом типа $(\mathscr{N}, \mathscr{M})$ назьвается унитарное отображение $U: \mathscr{M} \otimes \mathscr{F} \rightarrow \mathscr{L}$, где $\mathscr{L} \subseteq \mathscr{N}$, а $\mathscr{F}$ - произвольное ненулевое унитарное пространство. Квантовое состояние $|\xi\rangle \in \mathscr{M}$ кодируется произвольным смешанньм состоянием $\rho \in \mathbf{D}(\mathscr{L}) \subseteq \mathbf{D}(\mathscr{N})$ таким, что $\operatorname{Tr}_{\mathscr{F}}\left(U^{\dagger} \rho U\right)=|\xi\rangle\langle\xi|$. Общий вид кодирующего преобразования определяется предложением 3.6. Конструкция неоднозначного квантового кода присутствует в доказательстве теоремы 8.3. (Впоследствии мы назовем эту конструкцию производныц.м кодом.)

Теперь рассмотрим самую общую ситуацию. Пусть $\mathscr{N}$ и $\mathscr{M}$ - комбинированные пространства. Неоднозначныцм комбинированным кодом типа $(\mathscr{N}, \mathscr{M})$ назьвается пара $(\mathscr{L}, \chi)$, где $\mathscr{L} \subseteq \mathscr{N}$ - комбинированное подпространство, а $\chi: \mathbf{L}(\mathscr{M}) \rightarrow \mathbf{L}(\mathscr{L})$ инъективньй гомоморфизм алгебр с инволюцией. ( $\chi$ можно также рассматривать как гомоморфизм $\mathbf{L}(\mathscr{M}) \rightarrow \mathbf{L}(\mathscr{N})$, не сохраняюший единицу.) Состояние $\gamma \in \mathbf{D}(\mathscr{M})$ кодируется произвольным состоянием $\rho \in \mathbf{D}(\mathscr{L}) \subseteq \mathbf{D}(\mathscr{N})$ таким, что $\chi^{*} \rho=\gamma$. В частном случае неоднозначного квантового кода $\chi: X \mapsto U\left(X \otimes 1_{\mathscr{F}}\right) U^{\dagger}$.

Говорят, что код $\left(\mathscr{L}^{\prime}, \chi^{\prime}\right)$ грубее кода $(\mathscr{L}, \chi)$, если $\mathscr{L}^{\prime} \supseteq \mathscr{L}$ и ограничение преобразования $\left(\chi^{\prime}\right)^{*}$ на подпространство $\mathbf{L}(\mathscr{L})$ совпадает с $\chi^{*}$. Это имеет место в том случае, если $\chi(A)=\Pi_{\mathscr{L}} \chi^{\prime}(A)=\chi^{\prime}(A) \Pi_{\mathscr{L}}$ для каждого $A \in \mathbf{L}(\mathscr{M})$.

TеОрема 8.4. Пусть $\chi: \mathbf{L}(\mathscr{M}) \rightarrow \mathbf{L}(\mathscr{L})$ - инбективный гомоморфизм алгебр с инволючией, а $T \in \mathbf{T}(\mathscr{M}, \mathscr{L})$ - допустимое преобразование. Преобразование $T$ является кодирующим (т.е. $\left.\chi^{*} T=I_{\mathscr{M}}\right)$ тогда и только тогда, когда

$$
T(A B)=\chi(A) T(B)=T(A) \chi(B) \quad \text { для любых } \quad A, B \in \mathbf{L}(\mathscr{M}) .
$$

ДокаЗАТЕЛЬСтво. Пусть $\mathscr{M}=\left(\mathscr{M}_{j}: j \in \Omega\right)$. Пространство $\mathscr{L}$ можно считать унитарным (достаточно заменить его на $\overline{\mathscr{L}}$ ). Гомоморфизм $\chi$ отождествляет $\mathscr{L}$ с $\bigoplus_{j} \mathscr{M}_{j} \otimes \mathscr{F}_{j}$, где $\mathscr{F}_{j}$ - ненулевые унитарные пространства (см. (10)). Преобразование $T$ является кодируюшим тогда и только тогда, когда оно является прямой суммой кодируюших преобразований $T_{j} \in \mathbf{T}\left(\mathscr{M}_{j}, \mathscr{M}_{j} \otimes \mathscr{F}_{j}\right)$. Согласно предложению 3.6 , $T_{j}: X \mapsto X \otimes \lambda_{j}$, где $\lambda_{j} \in \mathbf{D}\left(\mathscr{F}_{j}\right)$. 
$\mathrm{C}$ другой стороны, формула (42) эквивалентна условию $T=\sum_{j} T_{j}, T_{j}: X \mapsto$ $X \otimes \lambda_{j}$, где $\lambda_{j} \in \mathbf{L}\left(\mathscr{F}_{j}\right)$. В действительности, $\lambda_{j} \in \mathbf{D}\left(\mathscr{F}_{j}\right)$, поскольку преобразование $T$ допустимое.

Теорема 8.3 легко обобщается на случай неоднозначных комбинированных кодов. Пусть $\mathscr{N}=\left(\mathscr{N}_{j}: j \in \Omega\right)$ и $\mathscr{N}^{\prime}=\left(\mathscr{N}_{k}^{\prime}: k \in \Omega^{\prime}\right)$ - комбинированные пространства, $(\mathscr{L}, \chi)$ - код типа $(\mathscr{N}, \mathscr{M})$, а $V_{j}: \mathscr{L}_{j} \rightarrow \mathscr{N}_{j}$ - естественные вложения. Рассмотрим пространство ошибок $\mathscr{E}=\bigoplus_{j, k} \mathscr{E}_{k j}\left(\mathscr{E}_{k j} \subseteq \mathbf{L}\left(\mathscr{N}_{j}, \mathscr{N}_{k}^{\prime}\right)\right)$. Введем обозначения: $\overline{\mathscr{L}^{\prime}}=$ $\mathscr{E} \overline{\mathscr{L}}\left(\mathscr{L}^{\prime} \subseteq \mathscr{N}^{\prime}\right.$ - комбинированное подпространство), $\mathscr{H}_{k j}=\mathscr{E}_{k j} V_{j} \subseteq \mathbf{L}\left(\mathscr{L}_{j}, \mathscr{L}_{k}^{\prime}\right)$,

$$
\mathbf{L}_{0}(\mathscr{L}, \chi)=\{A \in \mathbf{L}(\mathscr{L}): \forall B \in \mathbf{L}(\mathscr{M})(A \chi(B)=\chi(B) A)\} .
$$

Тогда вьполнены условия следующей теоремы.

TeOpema 8.5. Пусть $\mathscr{L}=\left(\mathscr{L}_{j}: j \in \Omega\right)$ u $\mathscr{L}^{\prime}=\left(\mathscr{L}_{k}^{\prime}: k \in \Omega^{\prime}\right)-\kappa о \mathcal{M} б$ нированные пространства, $\overline{\mathscr{H}}=\bigoplus_{j, k} \mathscr{H}_{k j}\left(\mathscr{H}_{k j} \subseteq \mathbf{L}\left(\mathscr{L}_{j}, \mathscr{L}_{k}^{\prime}\right)\right)$, причем $\overline{\mathscr{L}^{\prime}}=$ $\overline{\mathscr{H} \mathscr{L}}$. Рассмотрим некоторое множество вполне положительных преобразований $\subseteq \mathbf{T}\left(\mathscr{L}, \mathscr{L}^{\prime}\right)$ такое, что $\Re \subseteq \bigoplus_{j, k} \mathscr{H}_{k j} \cdot \mathscr{H}_{k j}^{*}$, причем пространства $\mathscr{H}_{k j}$ в этом условии не могут быть уменьшены. Пусть также $\chi: \mathbf{L}(\mathscr{M}) \rightarrow \mathbf{L}(\mathscr{L})-$ гомоморфизм алгебр с инволючией.

Следующие условия әквивалентны:

1) существует допустимое преобразование $P \in \mathbf{T}\left(\mathscr{L}^{\prime}, \mathscr{M}\right)$ и функиия с $: \Re \rightarrow$ $\mathbf{L}_{0}(\mathscr{L}, \chi)$ такие, ито РТ $\rho=\chi^{*}(c(T) \rho)$ для любых $T \in \Re, \rho \in \mathbf{L}(\mathscr{L}) ;$

2) существует гомоморфизм алгебр с инволюиией $\chi^{\prime}: \mathbf{L}(\mathscr{M}) \rightarrow \mathbf{L}\left(\mathscr{L}^{\prime}\right)$ такой, что $X \chi(A)=\chi^{\prime}(A) X, \chi(A) X^{\dagger}=X^{\dagger} \chi^{\prime}(A)$ для любьи $X \in \overline{\mathscr{H}}, A \in$ $\mathbf{L}(\mathscr{M})$

3) $Y^{\dagger} X \in \mathbf{L}_{0}(\overline{\mathscr{L}}, \chi)$ для любых $X, Y \in \overline{\mathscr{H}}$.

При этом $P$ и $\chi^{\prime}$ определены однозначно и не зависят от $\Re ; P=\left(\chi^{\prime}\right)^{*}$.

ДокАЗАТЕльСТво. Функция $c$ определена однозначно: $c(T)=T^{*} 1 \mathscr{L}^{\prime} \in \mathbf{L}(\mathscr{L})$ для каждого $T \in \mathbf{T}\left(\mathscr{L}, \mathscr{L}^{\prime}\right)$. Без уменьшения обшности, $\Re$ - выпукльй конус в пространстве $\mathbf{T}\left(\mathscr{L}, \mathscr{L}^{\prime}\right)$. Пусть $R \in \Re$ - произвольная точка из относительной внутренности этого конуса. Тогда $R=\sum_{j, k, s} X_{k j s} \cdot X_{k j s}^{\dagger}$, где операторы $X_{k j s}$ образуют базис пространства $\mathscr{H}_{k j}$. Кроме того, оператор $E=R 1_{\mathscr{L}}$ невырожден, поскольку $\overline{\mathscr{L}^{\prime}}=\overline{\mathscr{H} \mathscr{L}}$.

$1) \Rightarrow 3)$. Для каждого комбинированного подпространства $\mathscr{K} \subseteq \mathscr{M}$ оператор $\chi\left(\Pi_{\mathscr{K}}\right)$ является проектором на некоторое комбинированное подпространство, обозначаемое через $\chi(\mathscr{K})$. Достаточно показать, что операторы вида $Y^{\dagger} X(X, Y \in \overline{\mathscr{H}})$ коммутируют с такими проекторами.

Пусть $\mathscr{M}=\mathscr{K}_{1} \oplus \mathscr{K}_{2}$, где $\mathscr{K}_{1}, \mathscr{K}_{2}$ - взаимно ортогональные комбинированные подпространства; $\mathscr{G}_{i}=\chi\left(\mathscr{K}_{i}\right), E_{i}=R \Pi_{\mathscr{G}_{i}}, H_{i}=P^{*} \Pi_{\mathscr{K}_{i}}(i=1,2)$. Тогда $\operatorname{Tr}\left(E_{1} H_{2}\right)=$ $\operatorname{Tr}\left(E_{2} H_{1}\right)=0$. Заметим, что $E_{i}, H_{i}$ - положительные эрмитовы операторы, причем $E_{1}+E_{2}=E, H_{1}+H_{2}=1_{\mathscr{L}}$. Следовательно, $H_{1}$ и $H_{2}$ являются проекторами на некоторые взаимно ортогональные подпространства $\mathscr{G}_{1}^{\prime}, \mathscr{G}_{2}^{\prime} \subseteq \mathscr{L}^{\prime}$, причем $E_{i}=H_{i} E=E H_{i}$. Отсюда, во-первых, вытекает единственность $P$. Во-вторых, $\overline{\mathscr{H}} \mathscr{G}_{i} \subseteq \mathscr{G}_{i}^{\prime}$, следовательно, операторы вида $Y^{\dagger} X(X, Y \in \overline{\mathscr{H}})$ коммутируют с проекторами П $\mathscr{G}_{i}$.

$3) \Rightarrow 2)$. Положим $\chi^{\prime}(A)=E^{-1}(R \chi(A))$ для каждого $A \in \mathbf{L}(\mathscr{M})$. Требуемые условия и единственность $\chi^{\prime}$ проверяются тривиально. 
2) $\Rightarrow 1)$. Для любых $B \in \mathbf{L}(\mathscr{M}), \rho \in \mathbf{L}(\mathscr{L}), T \in \overline{\mathscr{H}} \cdot \overline{\mathscr{H}}^{*}$

$$
T(\chi(\mathrm{B}) \rho)=\chi^{\prime}(B)(T \rho), \quad T(\rho \chi(B))=(T \rho) \chi^{\prime}(B) .
$$

Следовательно,

$$
\operatorname{Tr}\left(\left(T^{*} 1_{\mathscr{L}^{\prime}}\right) \chi(B) \rho\right)=\operatorname{Tr}\left(\chi^{\prime}(B)(T \rho)\right)=\operatorname{Tr}\left(\left(T^{*} 1_{\mathscr{L}^{\prime}}\right) \rho \chi(B)\right),
$$

поэтому оператор $T^{*} 1_{\mathscr{L}^{\prime}}=c(T)$ коммутирует с $\chi(B)$. Если $P=\left(\chi^{\prime}\right)^{*}$, то

$$
(P T \rho, B)=\operatorname{Tr}\left((T \rho) \chi^{\prime}(B)\right)=\operatorname{Tr} T(\rho \chi(B))=\operatorname{Tr}(c(T) \rho \chi(B))=\left(\chi^{*}(c(T) \rho), B\right) .
$$

Вернемся к ситуации, описанной перед теоремой 8.5. Как правило, мы будем предполагать, что $\mathscr{N}^{\prime}=\mathscr{N}, \mathscr{E} \ni 1_{\mathscr{N}}$. В этом случае гомоморфизм $\chi^{\prime}$ инъективен. Код $\left(\mathscr{L}^{\prime}, \chi^{\prime}\right)$ называется производныцм кодом и обозначается через $\operatorname{Der}((\mathscr{L}, \chi), \mathscr{E})$. Например, $\operatorname{REP}(n, l)=\operatorname{Der}(\operatorname{REP}(n), \mathscr{E}(n, l))$. Производные коды обладают следуюшими очевидными свойствами.

1. Если $\mathscr{E}_{2} \supseteq \mathscr{E}_{1}$, то код $\operatorname{Der}\left(C, \mathscr{E}_{2}\right)$ грубее, чем код $\operatorname{Der}\left(C, \mathscr{E}_{1}\right)$.

2. $\operatorname{Der}\left(C, \mathscr{E}_{2} \mathscr{E}_{1}\right)=\operatorname{Der}\left(\operatorname{Der}\left(C, \mathscr{E}_{1}\right), \mathscr{E}_{2}\right)$.

3. Пусть $C=(\mathscr{L}, \chi)$ - код типа $(\mathscr{M}, \mathscr{K} \otimes \mathscr{F})$, исправляюший ошибки из $\mathbf{L}(\mathscr{F})$. Тогда $\operatorname{Der}(C, \mathbf{L}(\mathscr{F}))=(\mathscr{G} \otimes \mathscr{F}, \mu \otimes 1 \mathscr{F})$, где $(\mathscr{G}, \mu)-$ некоторый код типа $(\mathscr{M}, \mathscr{K})$.

\section{9. Симплектические (аддитивные) коды}

9.1. Алгебраическая подготовка. Рассмотрим пространство состояний одного кубита $\mathscr{B}=\overline{\mathbf{B}}$. В пространстве операторов $\mathbf{L}(\mathscr{B})$ имеется стандартньй базис, состояший из единичного оператра $1_{\mathscr{B}}=\sigma_{0}=\sigma_{00}$ и трех матриц Паули $\sigma_{x}=\sigma_{10}$, $\sigma_{y}=\sigma_{11}, \sigma_{z}=\sigma_{01}$ (см. (7)). Замечательные свойства этого базиса проявляются, если отождествить множество индексов $G=\{0, x, y, z\}=\{00,10,11,01\}$ с группой $\mathbf{Z}_{2} \times \mathbf{Z}_{2}$. Тогда

$$
\sigma_{\alpha \beta} \sigma_{\alpha^{\prime} \beta^{\prime}}=(-\mathrm{i})^{\alpha \beta^{\prime}-\beta \alpha^{\prime}} \sigma_{\alpha+\alpha^{\prime}, \beta+\beta^{\prime}}=(-1)^{\alpha \beta^{\prime}-\beta \alpha^{\prime}} \sigma_{\alpha^{\prime}, \beta^{\prime}} \sigma_{\alpha, \beta} .
$$

Таким образом, алгебра операторов $\mathbf{L}(\mathscr{B})$ наделена $G$-градуировкой $\left(G=\mathbf{Z}_{2} \times \mathbf{Z}_{2}\right)$. (Обобщение: Пусть $E$ - любая конечная абелева группа, $E^{*}$ - соответствуюшая группа характеров. Тогда алгебра $\mathbf{L}(\bar{E})$ наделена $\left(E \times E^{*}\right)$-градуировкой.)

Операторы вида $\sigma\left(\alpha_{1} \beta_{1}, \ldots, \alpha_{n} \beta_{n}\right)=\sigma_{\alpha_{1} \beta_{1}} \otimes \cdots \otimes \sigma_{\alpha_{n} \beta_{n}}$ образуют стандартный базис в пространстве $\mathbf{L}(\mathscr{B} \otimes n)$. Все они являются унитарными и эрмитовыми одновременно; их собственные числа равны \pm 1 . Порожденные этими операторами одномерные подпространства $(\sigma(f)): \sigma \in G^{n}$ задают $G^{n}$-градуировку алгебры $\mathbf{L}\left(\mathscr{B}^{\otimes n}\right)$. Эта градуировка согласована с инволюцией, т.е. если $X \in(\sigma(f))$, то $X^{\dagger} \in(\sigma(-f))$.

На группе $G^{n}$ (которую можно рассматривать как $2 n$-мерное пространство над полем $\mathbf{F}_{2}$ ) задана кососимметрическая форма

$$
\omega\left(\left(\alpha_{1} \beta_{1}, \ldots, \alpha_{n} \beta_{n}\right),\left(\alpha_{1}^{\prime} \beta_{1}^{\prime}, \ldots, \alpha_{n}^{\prime} \beta_{n}^{\prime}\right)\right)=\sum_{j=1}^{n} \alpha_{j} \beta_{j}^{\prime}-\beta_{j} \alpha_{j}^{\prime} \quad(\bmod 2),
$$


определяюшая коммутационные соотношения между базисными операторами:

$$
\sigma(f) \sigma(g)=(-1)^{\omega(f, g)} \sigma(g) \sigma(f)
$$

Автоморфизмы группы $G^{n}$, сохраняюшие форму $\omega$, образуют группу $\operatorname{Sp}_{2}(n)$ (симплектическую группу над полем $\left.\mathbf{F}_{2}\right)$. В дальнейшем важную роль будет играть группа $\operatorname{ESp}_{2}(n) \subseteq \mathbf{U T}\left(\mathscr{B}^{\otimes n}\right)$ унитарных преобразований, сохраняющих $G^{n}$-градуировку алгебры $\mathbf{L}\left(\mathscr{B}^{\otimes n}\right)$. Образ естественного гомоморфизма $\theta: \operatorname{ESp}_{2}(n) \rightarrow \operatorname{Aut}\left(G^{n}\right)$ равен $\mathrm{Sp}_{2}(n)$, а ядро состоит из преобразований вида $\sigma(f) \cdot \sigma(f)^{\dagger}: f \in G^{n}$. Следовательно, $\operatorname{ESp}_{2}(n) / G^{n} \cong \operatorname{Sp}_{2}(n)$, поэтому мы называемгруппу $\operatorname{ESp}_{2}(n)$ расширенной симплектической әруппой. Она не является просто полупрямым произведением груп $\operatorname{Sp}_{2}(n)$ и $G^{n}$. Группа $\operatorname{ESp}_{2}(1)$ изоморфна группе собственных вращений куба (см. раздел 4.2). Заметим, что элементам подгруппы $G$ отвечают повороты на угол $\pi$ вокруг координатных осей, а также тождественное преобразование. Нетрудно показать, что группа $\mathrm{ESp}_{2}(n)$ порождается унитарными операторами $S, K$ и $\widehat{\oplus}$, которые можно применять к различным кубитам. Оператор $\widehat{\oplus}$ следуюшим образом действует на канонические образуюшие алгебры $\mathbf{L}\left(\mathscr{B}^{\otimes 2}\right)$ :

$$
(\widehat{\oplus} \cdot \widehat{\oplus})[1,2]:\left(\sigma_{z}[1], \sigma_{z}[2], \sigma_{x}[1], \sigma_{x}[2]\right) \mapsto\left(\sigma_{z}[1], \sigma_{z}[1] \sigma_{z}[2], \sigma_{x}[1] \sigma_{x}[2], \sigma_{x}[2]\right) .
$$

Каноническими образующими $G^{n}$-градуированной алгебры с инволющией $\mathscr{G} \cong$ $\mathbf{L}\left(\mathscr{B}^{\otimes n}\right)$ называются образы операторов $\sigma_{x}[k], \sigma_{z}[k](k=1, \ldots, n)$ при произвольном изоморфизме $\Upsilon: \mathbf{L}\left(\mathscr{B}^{\otimes n}\right) \rightarrow \mathscr{G}$. Набор канонических образующих $Y_{\gamma k}=\Upsilon \sigma_{\gamma}[k]$ $(\gamma=x, z, k=1, \ldots, n)$ однозначно характеризует изоморфизм $\Upsilon$. Канонические образующие унитарны, принадлежат подпространствам градуировки и обладают следуюшими свойствами:

$$
Y_{\gamma k}^{2}=1, \quad Y_{x k} Y_{x l}=Y_{x l} Y_{x k}, \quad Y_{z k} Y_{z l}=Y_{z l} Y_{z k}, \quad Y_{x k} Y_{z l}=(-1)^{\delta_{k l}} Y_{z l} Y_{x k}
$$

Обратимые линейные отображения вида $u: \mathbf{F}_{2}^{n} \rightarrow \mathbf{F}_{2}^{n}$ образуют линейную груп$n y \mathrm{GL}_{2}(n)$. Каждому такому отображению соответствует оператор $\widehat{u} \in \mathbf{U}\left(\mathscr{B}{ }^{\otimes n}\right)$ и преобразование $\widehat{u} \cdot \widehat{u}^{\dagger} \in \mathrm{ESp}_{2}(n)$. Таким образом, $\mathrm{GL}_{2}(n) \subseteq \mathrm{ESp}_{2}(n)$. Группа $\mathrm{GL}_{2}(n)$ порождается оператором $\widehat{\oplus}$, которьй можно применять к различным кубитам.

Градуированные подалгебры с инволюцией алгебры $\mathbf{L}\left(\mathscr{B}{ }^{\otimes n}\right)$ соответствуют линейньм подпространствам $F \subseteq G^{n}$ и обозначаются через $\sigma(F)$. Подалгебра $\sigma(F)$ коммутативна тогда и только тогда, когда подпространство $F$ является однороднылм, т.е. $\omega(f, g)=0$ для любых $f, g \in G^{n}$. Действуя подходяшим симплектическим преобразованием, любое однородное подпространство $F \subseteq G^{n}$ можно привести к стандартному виду

$$
F^{\prime}=\left\{\left(0 \beta_{1}, \ldots, 0 \beta_{s}, 0, \ldots, 0\right): \beta_{1}, \ldots, \beta_{s} \in \mathbf{F}_{2}\right\}
$$

где $s=\operatorname{dim} F$. Образуюшими алгебры $\sigma\left(F^{\prime}\right)$ являются операторы $\sigma_{z}[1], \ldots, \sigma_{z}[s]$.

9.2. Основная конструкция. Пусть $n=s+m$. Определим стандартное вложение $V^{\prime}: \mathscr{B}^{\otimes m} \rightarrow \mathscr{B}^{\otimes n}$ по формуле $|\xi\rangle \mapsto|\underbrace{0, \ldots, 0}_{s}, \xi\rangle$. 
ОПРЕДЕЛЕНИЕ 9.1. Симплектическим (или аддитивным. ) кодом типа $(n, m)$ называется унитарное вложение вида $V=U V^{\prime}: \mathscr{B}^{\otimes m} \rightarrow \mathscr{B}^{\otimes n}$, где $U \cdot U^{\dagger} \in \operatorname{ESp}_{2}(n)$. Если $U=\widehat{u} S[1] \cdots S[l]$, где $u \in \mathrm{GL}_{2}(n), 0 \leqslant l \leqslant s$, то код назьвается квазилинейнылм.

Симплектические коды впервые были определены в статье [29]. Квазилинейные коды (в несколько иных терминах) рассматривали Steane [20], Shor и Calderbank [19].

Определение 9.1 конструктивно, но не очень удобно. Опишем симплектические коды в терминах алгебры $\mathbf{L}\left(\mathscr{B}{ }^{\otimes n}\right)$.

Характеристическим подпространством кода $V$ назьвается однородное подпространство $F=u F^{\prime} \subseteq G^{n}$, где $\mathrm{e}^{12} u=\theta\left(U \cdot U^{\dagger}\right) \in \operatorname{Sp}_{2}(n)$, а $F^{\prime}$ - стандартное однородное пространство (48). Легко видеть, что информационное подпространство $\mathscr{L}=V \mathscr{B}^{\otimes m} \subseteq \mathscr{B}^{\otimes n}$ является обшим собственным подпространством для всех операторов $\sigma(f): f \in F$. Таким образом, сушествует гомоморфизм алгебр с инволющией $\Phi: \sigma(F) \rightarrow \mathbf{C}$ такой, что

$$
\mathscr{L}=\left\{|\xi\rangle \in \mathscr{B}^{\otimes n}: \forall X \in \sigma(F)(X|\xi\rangle=\Phi(X)|\xi\rangle)\right\}
$$

Очевидно, что $\Phi(\sigma(f))=(-1)^{\varphi(f)}$, где $\varphi(f) \in \mathbf{F}_{2}$. Гомоморфизм $\Phi$ задается набором чисел $\varphi_{j}=\varphi\left(f_{j}\right) \in \mathbf{F}_{2}(j=1, \ldots, s)$, где $\left\{f_{1}, \ldots, f_{s}\right\}$ - произвольный базис подпространства $F$. Векторы $f_{j}$ и операторы $X_{j}=(-1)^{\varphi_{j}} \sigma\left(f_{j}\right)$ назьваются проверочнылми, поскольку измерение собственных значений операторов $X_{j}$ позволяет проверить принадлежность квантового состояния подпространству $\mathscr{L}$ :

$$
\mathscr{L}=\left\{|\xi\rangle \in \mathscr{B}^{\otimes n}: X_{j}|\xi\rangle=|\xi\rangle(j=1, \ldots, s)\right\}
$$

В некоторых случаях бьвает удобно определить избыточный набор проверочных операторов (см. раздел 9.4).

Симплектический кодполностью характеризуется заданием проверочных операторов $X_{1}, \ldots, X_{s}$ и информачионных операторов $Y_{\gamma k}=\left(U \cdot U^{\dagger}\right) \sigma_{\gamma}[s+k]$, где $\gamma=x, z$, $k=1, \ldots, m$. Очевидно, что $Y_{\gamma k}= \pm \sigma\left(g_{\gamma k}\right)$, причем $g_{\gamma k}$ принадлежат подпространству

$$
F_{+}=\left\{g \in G^{n}: \forall f \in F(\omega(f, g)=0)\right\} \supseteq F
$$

Информационные операторы определены с точностью до умножения на проверочные. (Неоднозначность возникает из-за того, что одному и тому же коду $V$ могут соответствовать различные операторы $U$.) На инвариантном язьке информационные операторы - это канонические образуюшие $F_{+} / F$-градуированной алгебры с инволюцией

$$
\sigma\left(F_{+}\right) /\left(\sigma\left(F_{+}\right) \operatorname{Ker} \Phi\right) \cong \mathbf{L}(\mathscr{L}) \cong \mathbf{L}\left(\mathscr{B}^{\otimes m}\right)
$$

Квазилинейные коды характеризуются двумя условиями:

1) каждый из операторов $X_{j}$ имеет вид $\sigma\left(\alpha_{1} 0, \ldots, \alpha_{n} 0\right)$ или $\sigma\left(0 \beta_{1}, \ldots, 0 \beta_{n}\right)$;

2) операторы $Y_{x k}$ имеют вид $\sigma\left(\alpha_{1} 0, \ldots, \alpha_{n} 0\right)$, а операторы $Y_{z k}$ имеют вид $\sigma\left(0 \beta_{1}, \ldots, 0 \beta_{n}\right)$.

\footnotetext{
${ }^{12}$ Напомним, что $\theta: \operatorname{ESp}_{2}(n) \rightarrow \operatorname{Aut}\left(G^{n}\right)-$ естественньй гомоморфизм.
} 
Перейдем к изучению исправляющих свойств симплектических кодов. Пусть пространство ошибок имеет вид $\sigma(E)$, где $E \subseteq G^{n}$ - произвольное множество. Для приложений интерес представляют пространства вида $\mathscr{E}(n, k)=\sigma(E(n, k))$, где

$$
\begin{gathered}
E(n, k)=\left\{g \in G^{n}:|\operatorname{Supp}(g)| \leqslant k\right\}, \\
\operatorname{Supp}\left(\alpha_{1} \beta_{1}, \ldots, \alpha_{n} \beta_{n}\right)=\left\{j: \alpha_{j} \neq 0 \text { или } \beta_{j} \neq 0\right\} .
\end{gathered}
$$

Для краткости ошибками будем назьвать как элементы множества $E$, так и соответствуюшие операторы $\sigma(g): g \in E$.

Обозначим через $F^{*}$ пространство линейных функционалов на $F$ со значениями в $\mathbf{F}_{2}$. Тогда

$$
\mathscr{B}^{\otimes n}=\bigoplus_{h \in F^{*}} \mathscr{L}_{h}, \quad \text { где } \mathscr{L}_{h}=\left\{|\xi\rangle \in \mathscr{B}^{\otimes n}: X_{j}|\xi\rangle=(-1)^{\left(h, f_{j}\right)}|\xi\rangle(j=1, \ldots, s)\right\},
$$

$$
\sigma(g) \mathscr{L}=\mathscr{L}_{\mu(g)}, \quad \mu(g)=\omega(\cdot, g) \in F^{*} \quad\left(\text { для любого } g \in G^{n}\right) .
$$

Очевидно, что $\mathscr{L}_{0}=\mathscr{L}$, а $\operatorname{Ker} \mu=F_{+}$. Функционал $\mu(g)$ удобно представить в координатной форме: $\mu(g)=\left(\mu_{1}(g), \ldots, \mu_{n}(g)\right)$, где $\mu_{j}(g)=\omega\left(f_{j}, g\right)$. По аналогии с классической теорией линейных кодов [17], $\mu(g)$ называют синдромом ошибки $g$.

Ошибки $g^{\prime}, g^{\prime \prime} \in E$ называются неразличимылми, если $\mu\left(g^{\prime}\right)=\mu\left(g^{\prime \prime}\right)$, т.е. $g^{\prime}-g^{\prime \prime} \in$ $F_{+}$. Ошибки неразличимы тогда и только тогда, когда $V^{\dagger} \sigma\left(g^{\prime}\right)^{\dagger} \sigma\left(g^{\prime \prime}\right) V \neq 0$. Ошибки $g^{\prime}, g^{\prime \prime} \in E$ назьваются эквивалентнылми, если $g^{\prime}-g^{\prime \prime} \in F$. Ошибки эквивалентны тогда и только тогда, когда они неразличимы и $V^{\dagger} \sigma\left(g^{\prime}\right)^{\dagger} \sigma\left(g^{\prime \prime}\right) V \in \mathbf{L}_{0}\left(\mathscr{B}{ }^{\otimes m}\right)$. Применительно к симплектическим кодам условие 3) теоремы 8.3 формулируется так: “любые две неразличимые ошибки эквивалентны”. Таким образом, доказана следующая

ТеОрема 9.2. Симплектический код $V$ исправляет ошибки из $\sigma(E)\left(E \subseteq G^{n}\right)$ тогда и только тогда, когда

$$
(E-E) \cap F_{+} \subseteq F, \quad \text { əде } \quad E-E=\left\{g^{\prime}-g^{\prime \prime}: g^{\prime}, g^{\prime \prime} \in E\right\}
$$

(Здесь $F \subseteq G^{n}$ - характеристическое подпространство кода $V$.)

СлеДСТВИЕ. Симплектический код исправляет $k$ ошибок тогда и только тогда, когда $E(n, 2 k) \cap F_{+} \subseteq F$.

Пример 9.3. Симплектический код типа $(5,1)$, исправляюший одну ошибку (cм. [21], [25], где тот же код определен в других терминах).

Пусть проверочные векторы $f_{1}, \ldots, f_{4}$ задаются строками следуюшей таблицы:

\begin{tabular}{|ll|ll|ll|ll|ll|}
\hline 1 & 0 & 1 & 0 & 0 & 1 & 0 & 1 & 0 & 0 \\
\hline 1 & 0 & 0 & 1 & 1 & 0 & 0 & 0 & 0 & 1 \\
\hline 0 & 1 & 0 & 0 & 0 & 1 & 1 & 0 & 0 & 1 \\
\hline 0 & 1 & 0 & 1 & 0 & 0 & 0 & 1 & 1 & 0 \\
\hline
\end{tabular}

Очевидно, что $\omega\left(f_{i}, f_{j}\right)=0(i, j=1,2,3,4)$. Любые две пары столбцов линейно независимы, следовательно, $E(4,2) \cup F_{+}=\{0\}$. 
9.3. Процедура исправления ошибок. Теорема 9.2 ничего не говорит о том, как реализовать исправляющее преобразование в виде схемы. Исправлять ошибки можно, например, таким образом. Допустим, что состояние $|\xi\rangle \in \mathscr{L}$ подверглось ошибке $g \in E$. Получилось состояние $|\psi\rangle=\sigma(g)|\xi\rangle$. С помощью измеряюших операторов $\Xi\left(X_{j}\right)$ (см. (31)) можно определить синдром ошибки $\mu(g)$. Благодаря условию (54) сама ошибка $g$ восстанавливается по синдрому однозначно, с точностью до эквивалентности. Действуя на вектор $|\psi\rangle$ оператором $\sigma\left(g^{\prime}\right)\left(g^{\prime}-g \in F\right)$, получаем вектор $\pm|\xi\rangle$. Ошибка исправлена.

Проведем это рассуждение более аккуратно. Пусть $\Delta-$ основной регистр (длины $n)$, a $\Delta^{\prime}$ и $\Delta^{\prime \prime}$ - вспомогательные регистры (длины $s$ и $2 n$, соответственно). Попробуем построить исправляющее преобразование $R \in \mathbf{T}(\mathscr{B} \otimes n)$ в виде композиции трех допустимых преобразований:

$$
R[\Delta]=\operatorname{CORR}\left[\Delta ; \Delta, \Delta^{\prime \prime}\right] \nu\left[\Delta^{\prime \prime} ; \Delta^{\prime}\right] \operatorname{MEAS}\left[\Delta, \Delta^{\prime} ; \Delta\right] .
$$

Здесь MEAS - процедура измерения синдрома, которая тоже состоит из трех стадий. Сначала во вспомогательный регистр $\Delta^{\prime}$ заносится состояние $|0\rangle$, затем применяются унитарные операторы $\Xi\left(X_{j}\right)[\Delta, j]$ (для $j \in\{1, \ldots, s\}=\Delta^{\prime}$ ), и, наконец, вспомогательные биты делаются классическими (с помошњю преобразования $Q_{\mathbf{B}}^{*}$ ). Преобразование $\mathrm{MEAS} \in \mathbf{T}\left(\mathscr{B} \otimes n, \mathscr{B}^{\otimes n} \times F^{*}\right)$ действует следующим образом (cp. с (16)):

$$
\operatorname{MEAS} \rho=\sum_{h \in F^{*}}\left(\Pi_{\mathscr{L}_{h}} \rho \Pi_{\mathscr{L}_{h}}, h\right) .
$$

Функция $\nu: F^{*} \rightarrow E \subseteq G^{n}$ вычисляет ошибку по синдрому. Точнее, $\nu(\mu(g))-g \in F$ для любого $g \in E$. Последнее преобразование $\mathrm{CORR} \in \mathbf{T}\left(\mathscr{B}^{\otimes n} \times G^{n}, \mathscr{B}^{\otimes n}\right)$ исправляет ошибку, т.е. $\operatorname{CORR}(\rho, g)=\sigma(g) \rho \sigma(g)$.

Построенное преобразование $R$ можно представить в виде

$$
R=\sum_{h \in F^{*}} W_{h} \cdot W_{h}^{\dagger}, \quad \text { где } W_{h}=\sigma(\nu(h)) \Pi_{\mathscr{L}_{h}} .
$$

Покажем, что оно действительно является исправляюшим. Пусть

$$
T=\sum_{f, g \in E} b_{f g} \sigma(f) \cdot \sigma(g)
$$

a $\rho \in \mathbf{L}(\mathscr{L})$. Тогда

$$
R T \rho=\sum_{\mu(f)=\mu(g)=h} b_{f g} \sigma(\nu(h)) \rho \sigma(\nu(h))=\left(\sum_{f, g \in E} c_{f g} b_{f g}\right) \rho
$$

(где $c_{f g} \in \mathbf{C}$ - константы), что и требовалось.

Преобразование CORR реализуется слоистой схемой глубины 2 из элементов $\Lambda_{c}\left(\sigma_{x}\right), \Lambda_{c}\left(\sigma_{z}\right)$. Функция $\nu$ реализуется некоторой схемой из классических элементов, однако размер и глубина этой схемы могут оказаться большими. Преобразование MEAS реализуется схемой глубины $O(n)$ и размера $O\left(n^{2}\right)$ из элементов $|0\rangle, S, \Lambda\left(\sigma_{x}\right)$, $\Lambda\left(\sigma_{y}\right), \Lambda\left(\sigma_{z}\right)$ и $Q_{\mathbf{B}}^{*}$.

Особый интерес в этой связи представляют коды с локальными проверками. Так называются бесконечные семейства симплектических кодов, у которых

а) проверочные векторы имеют носители ограниченного размера;

б) каждый кубит входит в ограниченное число таких носителей;

в) число исправляемых ошибок не ограничено. 
Для кодов с локальными проверками преобразование MEAS реализуется схемой глубины $O(1)$ и размера $O(n)$. Таким образом, если при работе схемы MEAS случится одна ошибка, то будет испорчено лишь $O(1)$ кубитов. ${ }^{13}$ Благодаря этому свойству частичное исправление ошибок можно реализовать схемой из неточных элементов, см. раздел 11.1. (Впрочем, метод Shor'a [43] позволяет это делать для произвольных симплектических кодов.)

9.4. Торические коды. Красивьм примером кодов с локальными проверками является бесконечная последовательность квазилинейных кодов $\operatorname{TOR}(k)(k=1,2, \ldots)$, которые строятся следуюшим образом [42].

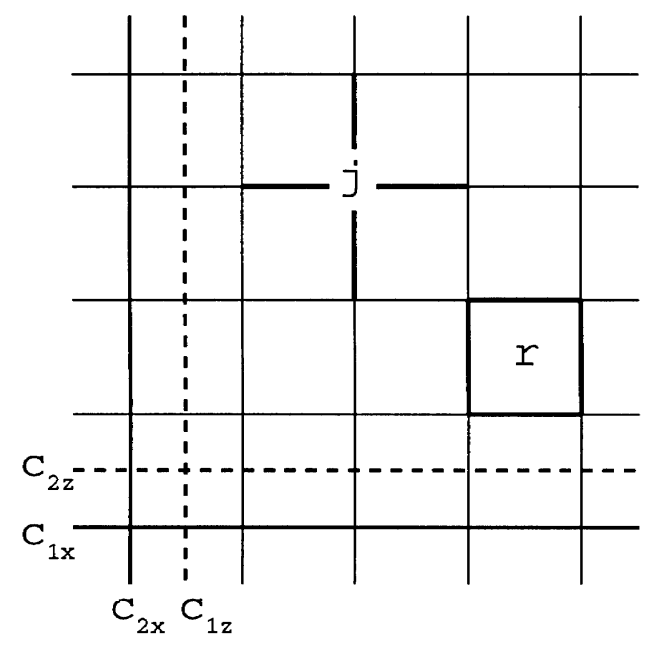

Рис. 1. Торический код $\operatorname{TOR}(5)$

Рассмотрим квадратную сетку размера $k \times k$ на торе (см. рис. 1 ). Пусть $A_{0}-$ множество вершин этой сетки, $A_{1}$ - множество ребер, $A_{2}$ - множество граней (двумерных клеток). Сопоставим каждому ребру по кубиту. (Всего имеется $n=2 k^{2}$ ребер.) Каждой грани $r \in A_{2}$ и каждой вершине $j \in A_{0}$ сопоставляются проверочные операторы

$$
X_{r}=\prod_{l \in \text { Граница }(r)} \sigma_{x}[l], \quad X_{j}=\prod_{l \in \text { Звезда }(j)} \sigma_{z}[l] .
$$

(Между этими операторами имеются два соотношения: $\prod_{r \in A_{2}} X_{r}=1$ и $\prod_{j \in A_{0}} X_{j}=1$.) Информационные операторы $Y_{x 1}, Y_{x 2}, Y_{z 1}, Y_{z 2}$ сопоставляются циклам $c_{x 1}, c_{x 2}$ и коциклам (разрезам) $c_{z 1}, c_{z 2}$ (см. рис. 1): нужно взять произведение операторов $\sigma_{x}[l]$ или $\sigma_{z}[l]$ по всем ребрам, входяшим в цикл или разрез. Построенньй таким образом торический код $\operatorname{TOR}(k)$ является квазилинейным кодом типа $\left(2 k^{2}, 2\right)$.

Чтобы убедиться в корректности этого определения, рассмотрим комплексы цепей $C_{0} \stackrel{\partial_{1}}{\longleftarrow} C_{1} \stackrel{\partial_{2}}{\longleftarrow} C_{2}$ и коцепей $C_{0}^{*} \stackrel{\partial_{1}^{*}}{\longrightarrow} C_{1}^{*} \stackrel{\partial_{2}^{*}}{\longrightarrow} C_{2}^{*}$ с коэффициентами в $\mathbf{Z}_{2}$. Отождествим группу $G^{n}$ с группой $C_{1} \oplus C_{1}^{*}$ согласно очевидному правилу

$$
G^{n} \ni\left(\alpha_{1} \beta_{1}, \ldots, \alpha_{n} \beta_{n}\right)=\left(\left(\alpha_{1}, \ldots, \alpha_{n}\right),\left(\beta_{1}, \ldots, \beta_{n}\right)\right) \in C_{1} \oplus C_{1}^{*} .
$$

\footnotetext{
${ }^{13} \mathrm{O}$ том, что такое “ошибка при работе схемы” см. раздел 10.
} 
Проверочные операторы (59) отвечают подпространству $F=\operatorname{Im} \partial_{2} \oplus \operatorname{Im} \partial_{1}^{*} \subseteq G^{n}$. Это подпространство однородно, поскольку $\left(\partial_{1}^{*} c_{0}^{\prime}, \partial_{2} c_{2}\right)=0$ для любых $c_{0}^{\prime} \in C_{0}^{*}$ и $c_{2} \in C_{2}$. Итак,

$$
F=\operatorname{Im} \partial_{2} \oplus \operatorname{Im} \partial_{1}^{*}, \quad F_{+}=\operatorname{Ker} \partial_{1} \oplus \operatorname{Ker} \partial_{2}^{*}, \quad F_{+} / F=H_{1} \oplus H_{1}^{*},
$$

где $H_{1} \cong H_{1}^{*} \cong\left(\mathbf{Z}^{2}\right)^{2}$-группы гомологий и когомологий тора с коэффициентами в $\mathbf{Z}_{2}$. Очевидно, что операторы $Y_{x 1}, Y_{x 2}, Y_{z 1}, Y_{z 2}$ являются каноническими образуюшими $F_{+} / F$-градуированной алгебры (51).

Носитель любого негомологичного нулю цикла или коцикла состоит из не менее чем $k$ ребер. Следовательно, код $\mathrm{TOR}(k)$ исправляет $\left\lfloor\frac{k-1}{2}\right\rfloor$ ошибок.

Исправление ошибок, т.е. вычисление функции $\nu$, сводится к поиску наименьшей 1-цепи по ее границе. В свою очередь, эта задача сводится к задаче о паросочетании наименьшего веса, для которой известен полиномиальньй алгоритм [55].

Данная конструкция имеет обобщение, основанное на алгебрах Хопфа [48].

\section{0. Исправление ошибок в процессе вычисления: общие принципы}

10.1. Определения и результаты. Конкретизируем задачу, поставленную в разделе 7: "Как моделировать схему с точными элементами схемой с неточньми элементами?" Трудности при формулировке этой задачи возникают уже в классическом случае.

Первое, что приходит в голову, - это потребовать, чтобы неточная схема реализовывала то же самое преобразование, что и точная. Однако это невозможно, так как любая ошибка на первом или последнем шаге неминуемо нарушит входную или выходную информацию. (Ошибки на промежуточных шагах не так страшны, так как к этому времени можно успеть защитить входную информацию с помощью какого-нибудь кода.) Единственньй способ обойти эту трудность - это подавать входную информацию в закодированном виде. Выходная информация тоже должна быть закодирована, причем с использованием неоднозначного кода. (Неоднозначность включает в себя ошибки, возникаюшие на последнем шаге.) Наконец, представляется естественным побитно кодировать все промежуточные результаты, получаюшиеся при работе точной схемы.

Таким образом, мы пришли к концепщии вычисления с закодированными битами (классическими или квантовыми). Вот формальное определение.

ОПРЕДЕЛЕНИЕ 10.1. Пусть $C=(\mathscr{L}, \chi)$ и $C^{\prime}=\left(\mathscr{L}^{\prime}, \chi^{\prime}\right)$ - неоднозначные комбинированные коды типа $(\mathscr{N}, \mathscr{M})$ и $\left(\mathscr{N}^{\prime}, \mathscr{M}^{\prime}\right)$, соответственно. Рассмотрим допустимые преобразования $T \in \mathbf{T}\left(\mathscr{N}, \mathscr{N}^{\prime}\right)$ и $P \in \mathbf{T}\left(\mathscr{M}, \mathscr{M}^{\prime}\right)$. Говорят, что $T$ представляет $P$ в кодах $C, C^{\prime}$, если для некоторого допустимого преобразования $R \in \mathbf{T}\left(\mathscr{L}, \mathscr{L}^{\prime}\right)$ имеет место коммутативная диаграмма

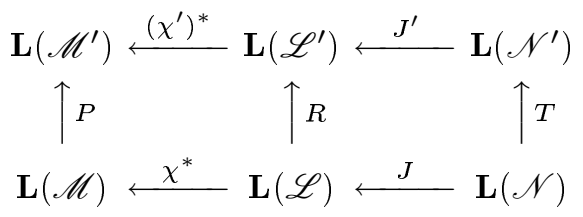


(Здесь $J, J^{\prime}$ - естественные вложения.) Если при этом $\operatorname{Im} T \subseteq \mathbf{L}\left(\mathscr{L}^{\prime}\right)$, т.е. можно еще добавить стрелку $\mathbf{L}(\mathscr{N}) \rightarrow \mathbf{L}\left(\mathscr{L}^{\prime}\right)$, то говорят, что $T$ представляет $P$ в сильном cмысле. Если коммутативность имеет место с точностью $\varepsilon$ (в смысле нормы $\|\cdot\| \diamond)$, то говорят, что $T$ представляет $P$ с точностью $\varepsilon$.

Заметим, что если $T_{1}, T_{2}$ представляют $P_{1}, P_{2}$ с точностью $\varepsilon_{1}, \varepsilon_{2}$, то произведение $T_{2} T_{1}$ представляет $P_{2} P_{1}$ с точностью $\varepsilon_{1}+\varepsilon_{2}$. Прежде чем двигаться дальше, полезно разобраться в некоторых тонкостях понятия "представляет с точностью $\varepsilon$ ". В классическом случае принятое нами определение является, по существу, единственно возMOжHЬLM.

ПРЕДЛОЖЕНИЕ 10.2. Пусть $P \in \mathbf{T}\left(M, M^{\prime}\right)$ u $\widetilde{T} \in \mathbf{T}\left(N, N^{\prime}\right)-$ классические допустимье преобразования. Предположим, что $\widetilde{T}$ представляет $P$ с точностью $\varepsilon$ (в каких-нибудь классических кодах). Тогда существует допустимое преобразование $T \in \mathbf{T}\left(N, N^{\prime}\right)$, представляющее $P$, точно такое, что $\| T-$ $\widetilde{T} \|_{\diamond} \leqslant 2 \varepsilon$.

(Доказательство оставляем читателю в качестве упражнения.) Квантовый аналог этого утверждения неверен, однако справедлива следующая

TeOpema 10.3. Пусть $P \in \mathbf{T}\left(\mathscr{M}, \mathscr{M}^{\prime}\right)$ u $\widetilde{T} \in \mathbf{T}\left(\mathscr{N}, \mathscr{N}^{\prime}\right)$ - доnустимые преоб-

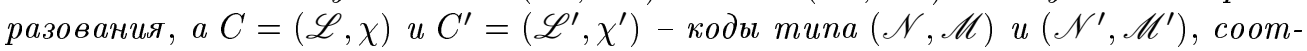
ветственно.

а) $\widetilde{T}$ представляет $P$ с точностью $O(\varepsilon)$ тогда и только тогда, когда существуют (не обязательно допустимье) преобразования $T$ и $R$ такие, что диаграмма (61) коммутативна, $и\|T-\widetilde{T}\|_{\diamond} \leqslant O(\varepsilon)$.

b) Если $\widetilde{T}$ представляет $P$ с точностью $\varepsilon$, то существует допустимое преобразование $T \in \mathbf{T}\left(\mathscr{N}, \mathscr{N}^{\prime}\right)$, представляющее $P$, точно такое, что $\|T-\widetilde{T}\|_{\diamond} \leqslant\left(\operatorname{dim} \mathscr{M}^{\prime}\right) O\left(\varepsilon^{1 / 2}\right)$.

Слово “представляет" можнн везде заменить на “представляет в сильном cммblсле".

(Доказательство будет приведено в разделе 10.2.)

В дальнейшем будут использоваться только коды типа $(n, 1)$. Для кодирования нескольких битов следует взять произведение таких кодов, т.е. каждый бит кодируется независимо.

Исправление классических ошибок. Предположим, что в нашем распоряжении имеются классические элементы из базиса $\mathscr{C}$, реализованные с точностью $\delta$. Покажем, как представить эти элементы с точностью $O\left(\delta^{2}\right)$ в коде $\operatorname{REP}(5,1)$. Последуюшие (не вполне строгие) рассуждения неявно опираются на предложение 10.2.

На рис. 2а изображена схема, реализующая исправляющее преобразование $R_{n} \in$ $\mathbf{T}\left(\mathbf{B}^{n}\right)$ для кода $\operatorname{REP}(n)$. Сначала каждьй бит копируется в $n$ экземплярах (с помошю некоторой схемы DUP). Затем берется по одному экземпляру каждого бита и вьчисляется функция МАЈ. Покажем, что при $n \geqslant 5$ возмущенное преобразование $\widetilde{R}_{n}$ представляет тождественное преобразование одного бита $I_{\mathbf{B}}$ в коде $\operatorname{REP}(n, 1)$ с точностью $O\left(\delta^{2}\right)$. Здесь мы имеем дело с классическими преобразованиями, поэтому "точность" означает "вероятность ошибки". 


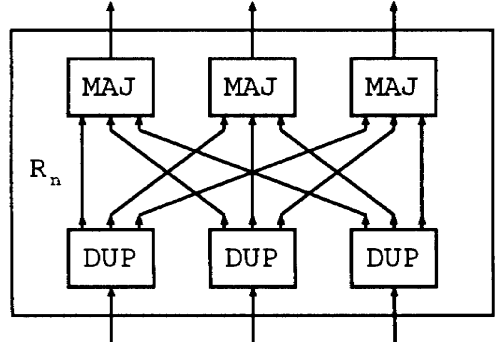

a)

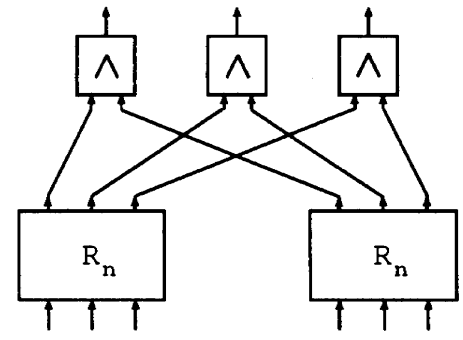

b)

Рис. 2. Классические схемы, устойчивые к ошибкам: а) схема $R_{n}$, реализующая исправляющее преобразование для кода $\operatorname{REP}(n)$; b) схема, реализующая операцию $\wedge$ в коде $\operatorname{REP}(n)$. (В данном случае $n=3$ )

Допустим, что по меншшей мере $n-1$ входных битов имеют значение $a$. С вероятностью $1-O\left(\delta^{2}\right)$ при работе схемы происходит не более одной ошибки. Если ошибка возникает при копировании какого-нибудь входного бита, то каждый из блоков МАЈ получит не более 2 неправильных входов. Следовательно, все выходы будут иметь значение $a$. Если же ошибка возникает при вычислении функции МАЈ, то на выходе будет не более одного неправильного бита.

Чтобы представить преобразование $\wedge$ в коде $\operatorname{REP}(n, 1)(n \geqslant 5)$, достаточно выполнить операцию $\wedge$ с каждой парой кодовых битов, предварительно исправив возможные ошибки на входе (см. рис. $2 \mathrm{~b}$ ). Если случится новая ошибка (в одной из подсхем $R_{n}$ или в одном из элементов $\wedge$ ), то будет испорчено не более одного выходного бита.

Элементы $\neg$ представляется аналогично, элемент 0 - тривиально.

Основная идея. Этот пример наводит на мысль, что можно последовательно повьшать точность вычислений, многократно подставляя код $\operatorname{REP}(5,1)$ в себя. Точность $O\left(\delta^{2^{r}}\right)$ обеспечивается некоторым кодом типа $\left(5^{r}, 1\right)$. (Такие коды назьваются каскадными.) Заметим, что размер получающихся схем растет как $a^{r}(a=$ const), т.е. как некоторая степень логарифма точности. Естественно, возникает желание обобщить эти рассуждения на квантовый случай. (Предполагается, что первый шаг - построение схем наподобие $R_{n}-$ уже сделан). Мы приступаем к реализации этого плана.

Теперь (наконец-то!) настало время сформулировать, в каком смысле схемы с точными элементами можно моделировать схемами с неточными элементами.

ОПРЕДЕЛЕНИЕ 10.4. Пусть $\mathscr{A}$ - некоторьй базис, элементы которого реализованы с фиксированной точностью $\delta$. Полиномиальной системой исправления ошибок называется совокупность следуюших объектов:

- последовательность кодов $\left(D_{k}: k=1,2, \ldots\right)$ типа $\left(n_{k}, 1\right)$ такая, что $n_{k}=$ $\operatorname{poly}(k)$;

- однородное полиномиальное семейство схем $\left(\Psi_{t k}: t \in \mathscr{A}, k=1,2, \ldots\right)$ в базисе $\mathscr{A}$; любое преобразование $T \in \operatorname{Trans}\left(\Psi_{t k}, \delta\right.$ ) (т.е. преобразование, реализуемое схемой $\Psi_{t k}$ с неточньми элементами) должно представлять преобразование $t$ в коде $D_{k}$ с точностью $\exp (-k)$;

- однородное полиномиальное семейство схем $\Upsilon_{k l}$ в базисе $\mathscr{A}$; схема $\Upsilon_{k l}$ (с неточньми элементами) должна представлять тождественное преобразование 
одного бита в кодах $D_{k}, D_{l}$ с точностью $\exp (-\min \{k, l\})$.

В случае комбинированных вычислений следует определить две последовательности кодов (для кодирования классических и квантовых битов, соответственно). Пока дело не дойдет до конкретных примеров, мы будем считать все биты квантовыми.

Оказьвается, что для построения полиномиальной системы исправления ошибок достаточно реализовать базисные элементы $t \in \mathscr{A}$ схемами $\Sigma_{t}$, устойчивыми к двум ошибкам ${ }^{14}$ (см. теорему 10.7 ниже). Грубо говоря, устойчивость означает, что схема $\Sigma_{t}$ продолжсает работать (т.е. представлять преобразование $t$ в определенных кодах), если произвольным образом изменить в ней любье два элемента. Однако это наивное определение не позволяет реализовать каскадную конструкцию, поскольку оно не допускает замены элементов схемы $\Sigma_{t}$ на их представления в некотором коде. "Правильное” определение устойчивости гораздо сложнее.

Введем несколько обозначений. Пусть $\Sigma$ - схема с множеством элементов $\Theta$. Для каждого $q \in \Theta$ определено множество выходов $\operatorname{Shade}(\Sigma, q)$, зависяших от элемента $q$. Если $A \subseteq \Theta$, то $\operatorname{Shade}(\Sigma, A)=\bigcup_{q \in A} \operatorname{Shade}(\Sigma, q)$.

Пусть $\Delta=\{1, \ldots, n\}, \Gamma \subseteq 2^{\Delta}$. По определению $2 \Gamma=\{A \cup B: A, B \in \Gamma\}$.

Cтупенчатой реализачией допустимого преобразования $P$ в кодах $C_{0}, C_{l}$ называется совокупность схем $\Sigma_{1}, \ldots, \Sigma_{l}$, допустимых преобразований $P_{1}, \ldots, P_{l}$ и кодов $C_{0}, C_{1}, \ldots, C_{l}$ такая, что:

(a) $T_{i}=\operatorname{Trans}\left(\Sigma_{i}\right)$ представляет $P_{i}$ в кодах $C_{i-1}, C_{i}$;

(б) $P_{l} \cdots P_{1}=P$.

Множество входов, выходов и элементов схемы $\Sigma_{i}$ обозначим через $\Delta_{i-1}, \Delta_{i}$ и $\Theta_{i}$, соответственно.

ОПРЕДЕЛЕНИЕ 10.5. Пусть $k \geqslant 0$ - целое число, $\Gamma \subseteq 2^{\Delta_{0}}, \Gamma^{\prime} \subseteq 2^{\Delta_{l}}$. Ступенчатая реализация назьвается $\left(k, \Gamma, \Gamma^{\prime}\right)$-устойчивой, если для любого $B_{0} \in \Gamma$ и $A=\bigcup_{i} A_{i}$ $\left(A_{i} \subseteq \Theta_{i}\right)$ такого, что $|A| \leqslant k$, найдутся $B_{1}, \ldots, B_{l}\left(B_{i} \subseteq \Delta_{i}\right)$ такие, что

1) код $C_{i}$ исправляет ошибки из $\mathscr{E}\left(B_{i}\right)(i=0, \ldots, l)$;

2 ) преобразование $T_{i}$ представляет $P_{i}$ в кодах $\operatorname{Der}\left(C_{i-1}, \mathscr{E}\left(B_{i-1}\right)\right), \operatorname{Der}\left(C_{i}, \mathscr{E}\left(B_{i}\right)\right)$;

3) $\operatorname{Shade}\left(\Sigma_{i}, A_{i}\right) \subseteq B_{i}$;

4) $B_{l} \in \Gamma^{\prime}$.

Для краткости ступенчатой реализащией иногда назьвается схема $\Sigma=\Sigma_{l} \circ \cdots \circ \Sigma_{1}$ (композиция схем $\left.\Sigma_{i}\right)$. Если $P \in \mathbf{T}\left(\mathscr{B}^{\otimes s}, \mathscr{B}^{\otimes m}\right)$ и используется побитное кодирование, то под $\left(k, \Gamma, \Gamma^{\prime}\right)$-устойчивой реализацией в коде $C$ подразумевается $\left(k, \Gamma^{s},\left(\Gamma^{\prime}\right)^{m}\right)$-устойчивая реализация в кодах $C^{s}, C^{m}$.

ПримеР 10.6. Схему $R_{n}$ (см. рис. $2 \mathrm{a}$ ) можно рассматривать как ступенчатую реализацию преобразования $I_{\mathbf{B}}$. Здесь $P_{1}=P_{2}=I_{\mathbf{B}}, C_{0}=C_{2}=\operatorname{REP}(n), C_{1}=\operatorname{REP}\left(n^{2}\right)$. Первая ступень $\left(\Sigma_{1}\right)$ состоит из подсхем DUP, вторая $\left(\Sigma_{2}\right)$ - из подсхем МАЈ. При $n \geqslant 5$ эта реализация является $(1, \Gamma, \Gamma)$-устойчивой, где $\Gamma=\{A \subseteq\{1, \ldots, n\}$ : $|A| \leqslant 1\}$. Более интересные примеры содержатся в разделе 11.

\footnotetext{
${ }^{14} \mathrm{~B}$ классическом случае хватило бы устойчивости к одной ошибке. Эта разница отражает различие между предложением 10.2 и теоремой 10.3 (b). Это и есть то самое тонкое место, где не годятся рассуждения "на физическом уровне строгости".
} 
Tеорема 10.7. Пусть $C$ - код типа $(n, 1)$, допускающий кодирование и декодирование схемами в базисе $\mathscr{A} ; \Gamma \subseteq 2^{\{1, \ldots, n\}}$. Предположим, что каждое преобразование $t \in \mathscr{A}$ обладает $(2,2 \Gamma, \Gamma)$-устойчивой ступенчатой реализацией $\Phi_{t}$ в коде $C$ в базисе $\mathscr{A}$. Кроме того, пусть $\operatorname{Trans}\left(\Phi_{I}\right)$ представляет тождественное преобразование $I=I_{\mathscr{B}}$ в сильном смысле. Тогда при достаточно малом $\delta>0$ в базисе $\mathscr{A}$ существует полиномиальная система исправления ошибок.

ЗАмЕчАниЕ. Если преобразование $t$ унитарное, то условие $(2,2 \Gamma, \Gamma)$-устойчивости можно заменить на $(2, \Gamma, \Gamma)$-устойчивости. Для классических схем достаточно потребовать $(1, \Gamma, \Gamma)$-устойчивость.

Таким образом, проблема возмущений свелась к поиску конечного объекта с определенньми свойствами.

\section{2. Доказательства.}

ДоКАЗАТЕЛЬСТво теОремы 10.3. а) Пусть $\widetilde{T}$ представляет $P$ с точностью $O(\varepsilon)$, т.е. имеет место коммутативная, с точностью $O(\varepsilon)$, диаграмма

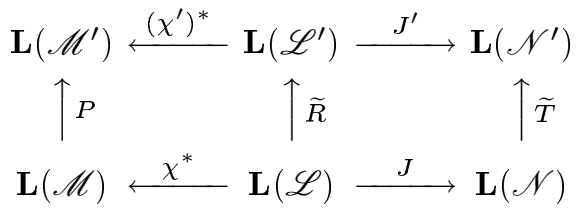

Положим $R=\widetilde{R}+H^{\prime}\left(P \chi^{*}-\left(\chi^{\prime}\right)^{*} \widetilde{R}\right), T=\widetilde{T}+\left(J^{\prime} R-\widetilde{T} J\right) J^{*}$, где $H^{\prime} \in \mathbf{T}\left(\mathscr{M}^{\prime}, \mathscr{L}^{\prime}\right)-$ произвольное кодируюшее преобразование для кода $C^{\prime}$, т.е. $\left(\chi^{\prime}\right)^{*} H^{\prime}=I_{\mathscr{M}^{\prime}}$.

Обратно, пусть имеет место коммутативная диаграмма $(61)$ и $\|T-\widetilde{T}\|_{\diamond} \leqslant O(\varepsilon)$. Положим

$$
\widetilde{R}=S\left(E^{-1 / 2} \cdot E^{-1 / 2}\right), \text { где } S=\left(J^{\prime}\right)^{*} \widetilde{T} J, \quad E=S^{*} 1_{\mathscr{L}^{\prime}} .
$$

Очевидно, что $\widetilde{R}$ - допустимое преобразование, причем $\|R-\widetilde{R}\|_{\diamond} \leqslant O(\varepsilon)$. Диаграмма (62) коммутативна с точностью $O(\varepsilon)$.

b) Для простоты будем считать, что все рассматриваемые пространства унитарные. В этом случае $\mathscr{L}^{\prime}=\mathscr{M}^{\prime} \otimes \mathscr{G}$ и $\left(\chi^{\prime}\right)^{*}=\operatorname{Tr} \mathscr{G}$. Кроме того, $J=A \cdot A^{\dagger}, J^{\prime}=B \cdot B^{\dagger}$, где $A: \mathscr{L} \rightarrow \mathscr{N}$ и $B: \mathscr{L}^{\prime} \rightarrow \mathscr{N}^{\prime}-$ естественные вложения.

Из приближенной коммутативности диаграммы (62) вытекают неравенства

$$
\left\|\left(\chi^{\prime}\right)^{*}\left(J^{\prime}\right)^{*} \widetilde{T} J-P \chi^{*}\right\|_{\diamond} \leqslant O(\varepsilon), \quad\left\|\left(B B^{\dagger} \otimes 1_{\mathscr{K}}\right) X A-X A\right\| \leqslant O(\varepsilon),
$$

где $X: \mathscr{N} \rightarrow \mathscr{N}^{\prime} \otimes \mathscr{K}$-каноническоепредставление преобразования $\widetilde{T}$. Найдем такое унитарное вложение $Y: \mathscr{L} \rightarrow \mathscr{N}^{\prime} \otimes \mathscr{K}$, что

$$
\left(\chi^{\prime}\right)^{*}\left(J^{\prime}\right)^{*} \operatorname{Tr}_{\mathscr{K}}\left(Y \cdot Y^{\dagger}\right)=P \chi^{*}, \quad\|X A-Y\| \leqslant O\left(\varepsilon^{1 / 2}\right) .
$$

Пусть $V, W: \mathscr{L} \rightarrow \mathscr{M}^{\prime} \otimes \mathscr{F}$ - канонические представления преобразований $\left(\chi^{\prime}\right)^{*}\left(J^{\prime}\right) * \widetilde{T} J$ и $P \chi^{*}$. Заметим, что оператор $\left(B^{\dagger} \otimes 1_{\mathscr{K}}\right) X A$ тоже является представлением преобразования $\left(\chi^{\prime}\right)^{*}\left(J^{\prime}\right) * \widetilde{T} J$. Из леммы 3.10 следует, что $\|W-V\| \leqslant O\left(\varepsilon^{1 / 2}\right)$. Согласно лемме 3.5 , сушествует унитарное вложение $U: \mathscr{F} \rightarrow \mathscr{G} \otimes \mathscr{K}$ такое, что $\left(B^{\dagger} \otimes 1_{\mathscr{K}}\right) X A=\left(1_{\mathscr{M}^{\prime}} \otimes U\right) V$. Теперь положим $Y=\left(B \otimes 1_{\mathscr{K}}\right)\left(1_{\mathscr{M}^{\prime}} \otimes U\right) W$. 
Для завершения доказательства осталось продолжить отображение $Y$ с подпространства $\mathscr{L} \subseteq \mathscr{N}$ на все пространство $\mathscr{N}$. Точнее говоря, нужно найти унитарное вложение $Z: \mathscr{N} \rightarrow \mathscr{N}^{\prime} \otimes \mathscr{K}$ такое, что $Y=Z A$ и $\|X-Z\| \leqslant O\left(\varepsilon^{1 / 2}\right)$. Тогда можно будет положить $T=\operatorname{Tr}_{\mathscr{K}}\left(Z \cdot Z^{\dagger}\right)$.

Пусть $\mathscr{L}_{\perp} \subseteq \mathscr{N}$ - ортогональное дополнение к $\mathscr{L}$, а $A_{\perp}: \mathscr{L}_{\perp} \rightarrow \mathscr{N}$ - соответствующее вложение. Положим $Z=Y A^{\dagger}+Y_{\perp} A_{\perp}^{\dagger}$, где

$$
Y_{\perp}=E\left(E^{\dagger} E\right)^{-1 / 2}, \quad E=\left(1-Y Y^{\dagger}\right) X A_{\perp}: \mathscr{L}_{\perp} \rightarrow \mathscr{N}^{\prime} \otimes \mathscr{K} .
$$

Очевидно, что $Z$ удовлетворяет требуемым свойствам.

Вариант теоремы с "представлением в сильном смысле" доказьвается аналогично.

Доказательству теоремы 10.7 мы предпошлем несколько лемм. Введем обозначение: $\mathscr{E}(\Gamma)=\sum_{A \in \Gamma} \mathscr{E}(A)$, где $\Gamma \subseteq 2^{\{1, \ldots, n\}}$.

Лемма 10.8. Пусть $T$ представляет $P$ в кодах $\operatorname{Der}(C, \mathscr{E}(B)), C^{\prime}$ для каждого $B \in 2 \Gamma$. Тогда $T$ представляет $P$ в кодах $\operatorname{Der}(C, \mathscr{E}(\Gamma)), C^{\prime} .($ (В классическом случае, а также если преобразование Р унитарное, $2 \Gamma$ можнн заменить на $Г$.)

ДоказАтельство. Пусть $C=(\mathscr{L}, \chi), C^{\prime}=\left(\mathscr{L}^{\prime}, \chi^{\prime}\right), \mathscr{L}_{B}=\mathscr{E}(B) \mathscr{L}, \mathscr{L}_{\Gamma}=$ $\mathscr{E}(\Gamma) \mathscr{L}$

Обший случай: $\mathscr{L}_{\Gamma}=\sum_{B \in \Gamma} \mathscr{L}_{B}$, поэтому $\mathbf{L}\left(\mathscr{L}_{\Gamma}\right) \subseteq \sum_{B \in 2 \Gamma} \mathbf{L}\left(\mathscr{L}_{B}\right)$. Дальше очевидно.

В классическом случае $\mathbf{L}\left(\mathscr{L}_{\Gamma}\right) \subseteq \sum_{B \in \Gamma} \mathbf{L}\left(\mathscr{L}_{B}\right)$.

Если преобразование $P$ унитарное, то можно считать, что $P=I_{\mathscr{M}}$. Пусть $T=$ $\sum_{s} X_{s} \cdot X_{s}^{\dagger}$, а $\mathscr{E}_{T}-$ пространство, порожденное операторами $X_{s}$. Для каждого $B \in \Gamma$ код $C_{B}=\operatorname{Der}(C, \mathscr{E}(B))$ исправляет ошибки из $\mathscr{E}_{T}$, а код $C^{\prime}$ грубее, чем $\operatorname{Der}\left(C_{B}, \mathscr{E}_{T}\right)=$ $\operatorname{Der}\left(C, \mathscr{E}_{T} \mathscr{E}(B)\right)$. Следовательно, гомоморфизм $\chi^{\prime}$ удовлетворяет условию 2) теоремы 8.5 , если положить $\overline{\mathscr{H}}=\mathscr{E}_{T} \mathscr{E}(B)$. Теперь можно взять сумму по $B \in \Gamma$ и обратить предыдушие рассуждения.

ЛЕмма 10.9. Пусть $\Sigma$ и $\widetilde{\Sigma}-$ схемы с одинаковой комбинаторной структурой, отличающиеся на множестве әлементов А. Предположим, что преобразование $T=\operatorname{Trans}(\Sigma)$ представляет $P$ в кодах $C, C^{\prime}$, причем код $C^{\prime}$ исправляет ошибки из $\mathscr{E}=\mathscr{E}(\operatorname{Shade}(\Sigma, A))$. Тогда $\widetilde{T}=\operatorname{Trans}(\widetilde{\Sigma})$ представляет $P$ в кодах $C$, $\operatorname{Der}\left(C^{\prime}, \mathscr{E}\right)$

ДокаЗательство. Пусть $P \in \mathbf{T}\left(\mathscr{M}, \mathscr{M}^{\prime}\right), T, \widetilde{T} \in \mathbf{T}\left(\mathscr{N}, \mathscr{N}^{\prime}\right), C=(\mathscr{L}, \chi)$, $C^{\prime}=\left(\mathscr{L}^{\prime}, \chi^{\prime}\right), \mathscr{F}$ - пространство состояний кубитов из множества $\operatorname{Shade}(\Sigma, A)$, а $\mathscr{K}$ - пространство состояний остальных выходов схемы $\Sigma$. Тогда $\operatorname{Der}\left(C^{\prime}, \mathscr{E}\right)=$ $(\mathscr{G} \otimes \mathscr{F}, \mu \otimes 1 \mathscr{F})$, где $(\mathscr{G}, \mu)$ - некоторый код типа $(\mathscr{M}, \mathscr{K})$. Очевидно, что $\operatorname{Tr}_{\mathscr{F}} \widetilde{T}=\operatorname{Tr}_{\mathscr{F}} T . \quad$ Если $\rho \in \mathbf{D}(\mathscr{L})$, то $T \rho \in \mathbf{D}\left(\mathscr{L}^{\prime}\right) \subseteq \mathbf{D}(\mathscr{G} \otimes \mathscr{F})$, поэтому $\operatorname{Tr}_{\mathscr{F}} \widetilde{T} \rho=\operatorname{Tr}_{\mathscr{F}} T \rho \in \mathbf{D}(\mathscr{G})$. Следовательно, $\widetilde{T} \rho \in \mathbf{D}(\mathscr{G} \otimes \mathscr{F})$ (здесь используется положительность преобразования $\widetilde{T})$. Очевидно, что $\mu^{*} \operatorname{Tr} \mathscr{F} \widetilde{T}=P \chi^{*}$.

Следуюшая лемма (как, впрочем, и предыдушие) верна для схем с обобщенными битами: пространство состояний каждого бита - произвольное комбинированное пространство. В данном случае важно, что получающаяся оценка не зависит от размерностей этих пространств. 
ЛЕмма 10.10. Пусть $\Sigma=\Sigma_{l} \circ \cdots \circ \Sigma_{1}$ есть $(k, 2 \Gamma, \Gamma)$-устойчивая ступенчатая реализачия преобразования $P$ в кодах $C, C^{\prime}$. Тогда любое преобразование $\widetilde{T} \in$ $\operatorname{Trans}(\Sigma, \delta)$ представляет $P$ в кодах $\operatorname{Der}(C, \mathscr{E}(\Gamma)), \operatorname{Der}\left(C^{\prime}, \mathscr{E}\left(\Gamma^{\prime}\right)\right)$ с точностью $b \delta^{k+1}$. Число b зависит только от размера схемь $\Sigma$. (В классическом случае, а также если преобразование $P$ унитарное, $2 \Gamma$ можнно заменить на Г.)

ДокАЗАТЕльство. Каждому элементу $q \in \Theta$ схемы $\Sigma$ сопоставим произвольное допустимое преобразование $\widetilde{T}_{q}$ такое, что $\left\|\widetilde{T}_{q}-T_{q}\right\|_{\diamond} \leqslant \delta$. Введем обозначение $T(A)=$ $\operatorname{Trans}(\Sigma(A))$, где $A \subseteq \Theta$, а схема $\Sigma(A)$ получена из $\Sigma$ заменой $T_{q} \mapsto \widetilde{T}_{q}$ для всех $q \in A$. Тогда $\widetilde{T}=T(\Theta)$.

Пусть $|A| \leqslant k$. Из определения устойчивой реализации и леммы 10.9 следует,что $T(A)$ представляет $P$ в кодах $\operatorname{Der}\left(C, \mathscr{E}\left(B_{0}\right)\right), \operatorname{Der}\left(C^{\prime}, \mathscr{E}\left(\Gamma^{\prime}\right)\right)$ для каждого $B_{0} \in 2 \Gamma$. Таким образом, $T(A)$ представляет $P$ в кодах $\operatorname{Der}(C, \mathscr{E}(\Gamma)), \operatorname{Der}\left(C^{\prime}, \mathscr{E}\left(\Gamma^{\prime}\right)\right)$ (см. лемму 10.8). Теперь воспользуемся тождеством

$$
\widetilde{T}=T(\Theta)=\sum_{E \subseteq \Theta} W(E), \quad \text { где } W(E)=\sum_{A \subseteq E}(-1)^{|E|-|A|} T(A) .
$$

Очевидно, что $\|W(E)\|_{\diamond} \leqslant \delta^{|E|}$. Положим $T=\sum_{|E| \leqslant k} W(E)$ и применим теоремy 10.3a).

Теперь введем важную конструкцию - подстановку одного кода в другой. Пусть $C=(\mathscr{L}, \chi)$ - код типа $(n, 1)$, а $D=(\mathscr{G}, \mu)$ - произвольньй код типа $(\mathscr{N}, \mathscr{B})$. Определим код $C \triangleleft D$ типа $(\mathscr{N} \otimes n, \mathscr{B})$ следующим образом: один (квантовый) бит представляется $n$ битами с помощью кода $C$, а затем каждњй из этих битов кодируется обобщенным битом (с пространством состояний $\mathscr{N}$ ) при помоши кода $D$. На формальном языке, $C \triangleleft D=(\mathscr{K}, \nu)$, где $\mathbf{L}(\mathscr{K})=\mu^{\otimes n} \mathbf{L}(\mathscr{L}), \nu=\mu^{\otimes n} \chi$.

Еще одно обозначение: $C \triangleleft_{\Gamma} D=\operatorname{Der}(C \triangleleft D, \mathscr{E}(\Gamma))$, где $\Gamma \subseteq 2^{\{1, \ldots, n\}}$. Подчеркнем, что код $C \triangleleft_{\Gamma} D$ грубее, чем код $\operatorname{Der}(C, \mathscr{E}(\Gamma)) \triangleleft D . \quad$ Если преобразование $T \in \mathbf{T}(\mathscr{N})$ представляет $I_{\mathscr{B}}$ в коде $D$ в сильном смысле, то $T^{\otimes n}$ представляет $I_{\mathscr{B}}$ в кодах $C \triangleleft_{\Gamma} D$, $\operatorname{Der}(C, \mathscr{E}(\Gamma)) \triangleleft D$ (также в сильном смысле).

ДОКАЗАТЕЛЬСТВО ТЕОРЕМЫ 10.7. Пусть $D_{0}$ - тождественньЙ код, $D_{r+1}=$ $C \triangleleft_{r} D_{r}(r=0,1, \ldots)$. (Коды $D_{r}$ назьваются каскадными.) Построим в базисе $\mathscr{A}$ схемы $\Psi_{t r}(t \in \mathscr{A})$ со следующим свойством: любое преобразование $\widetilde{T} \in \operatorname{Trans}\left(\Psi_{t r}, \delta\right)$ представляет $t$ в коде $D_{r}$ с точностью $\varepsilon_{r}=c \delta^{(3 / 2)^{r}}$, где $c=$ const. Kроме того, потребуем, чтобы любое $\widetilde{T} \in \operatorname{Trans}\left(\Psi_{I r}, \delta\right)$ представляло $I$ в коде $D$ в сильном смысле (с той же точностью).

Выберем число $c$ достаточно большим. Схема $\Psi_{t 0}$ будет состоять из одного элемента $t$. Индукция по $r:$ предположим, что все схемы $\Psi_{u r}: u \in \mathscr{A}$ уже построены. Схема $\Psi_{t, r+1}$ строится на основе ступенчатой реализации $\Phi_{t}=\Sigma=\Sigma_{l} \circ \cdots \circ \Sigma_{1}$ преобразования $t$. А именно, $\Psi_{t, r+1}=\Psi$ (если $\left.t \neq I\right)$, либо $\Psi_{t, r+1}=\Psi \circ \Psi \circ \Psi($ если $t=I)$, где

$$
\Psi=\Sigma^{\prime} \triangleleft \Psi_{* r}, \quad \Sigma^{\prime}=\Sigma_{l} \circ I^{s_{l-1}} \circ \cdots \circ \Sigma_{1} \circ I^{s_{0}} .
$$

Здесь $s_{i-1}=\left|\Delta_{i-1}\right|-$ число входов схемы $\Sigma_{i}$. Символ $\triangleleft \Psi_{* r}$ обозначает операцию замены всех элементов схемы (в данном случае, $\Sigma^{\prime}$ ) по правилу $u \mapsto \Psi_{u r}$. Покажем, что любое преобразование $\widetilde{T} \in \operatorname{Trans}(\Psi, \delta)$ представляет $T$ в коде $D_{r+1}$ с точностью $\varepsilon_{r+1}$. 
Преобразование $\widetilde{T}$ можно описать следуюшим образом. Заменим каждый элемент $q \in \Theta^{\prime}$ схемы $\Sigma^{\prime}$ (точнее говоря, соответствующее преобразование $u_{q} \in \mathscr{A}$ ) на произвольное преобразование $\widetilde{T}_{q} \in \operatorname{Trans}\left(\Psi_{u_{q}, r}, \delta\right)$. Если обозначить получившуюся схему через $\widetilde{\Omega}$, то $\widetilde{T}=\operatorname{Trans}(\widetilde{\Omega})$. (Схема $\widetilde{\Omega}$ работает с обобщенными битами, каждый из которых состоит из $n^{r}$ обычных битов.) Для каждого $q \in \Theta^{\prime}$ существует допустимое преобразование $T_{q}$, представляющее $u_{q}$ в коде $D_{r}$, такое, что $\left\|T_{q}-\widetilde{T}_{q}\right\|_{\diamond} \leqslant O\left(\varepsilon_{r}^{1 / 2}\right)$. (Применяя теорему $10.3 \mathrm{~b}$ ), мы учли, что $\operatorname{dim} \mathscr{M}^{\prime}=2^{m} \leqslant$ const, где $m$ - число выходов элемента $q$.) Если $u_{q}=I$, то $T_{q}$ представляет $I$ в сильном смысле. Заменяя $\widetilde{T}_{q}$ на $T_{q}$, мы получаем новую схему $\Omega$. Она является $(2,2 \Gamma, \Gamma)$-устойчивой ступенчатой реализацией преобразования $t$ в коде $C \triangleleft D_{r}$. Теперь остается воспользоваться леммой 10.10.

Если $t=I$, то $\operatorname{Trans}(\Omega)$ представляет $I$ в коде $D_{r+1}$ в сильном смысле. Следовательно, $\widetilde{T} \in \operatorname{Trans}(\widetilde{\Omega})$ представляет $I$ в коде $D_{r+1}$ в сильном смысле с точностью $O\left(\varepsilon_{r}^{1 / 2}\right)$. Замена $\Psi$ на $\Psi \circ \Psi \circ \Psi$ повьшает эту точность до $\varepsilon_{r+1}=O\left(\varepsilon_{r}^{3 / 2}\right)$.

Итак, первые два условия из определения 10.4 выполнены (с точностью до замены $k$ на $r \sim \log k)$. Что касается последнего условия, положим $\Upsilon_{r, r+1}=\Sigma \rightarrow \triangleleft \Psi_{* r}$, $\Upsilon_{r+1, r}=\Sigma_{\leftarrow} \triangleleft \Psi_{* r}$, где $\Sigma_{\rightarrow}$ и $\Sigma_{\leftarrow}-$ кодирующая и декодирующая схемы для кода $C$. Произвольная схема $\Upsilon_{k l}$ строится как композиция нескольких схем $\Upsilon_{r, r+1}$ или $\Upsilon_{r+1, r}$.

\section{1. Исправление ошибок: конкретные процедуры}

Теперь мы в состоянии доказать основной результат.

ТЕОРема 11.1. При достаточно малом $\delta>0$ в симплекто-классическом базисе $\mathscr{S} C$ и в полном базисе $\mathscr{Q} C$ существуют полиномиальнье системы исправления ошибок.

Симплекто-классический вариант этой теоремы был доказан автором независимо, тогда как при реализации элемента Тоффоли используется идея из работы Shor'a [43] .

Доказательство вполне конструктивно, однако степень полинома в полиномиальной системе получается довольно большой; мы даже не станем ее вьписывать. Теорема 11.1 демонстрирует принципиальную возможность построения надежных квантовых схем из ненадежных (неточных) элементов. Над практически пригодными процедурами исправления ошибок еще предстоит работать.

11.1. Симплекто-классический случай. В предыдущем разделе было показано, как исправлять ошибки при классических вычислениях. Теперь мы будем предполагать, что все классические элементы базиса $\mathscr{S} C$ - точные. (Иначе пришлось бы включить исправление классических ошибок в каждую из описьваемых ниже процедур.) Доказательство теоремы 11.1 основано на теореме 10.7. Чтобы не погрязнуть в деталях, мы будем апеллировать к интуитивному представлению об устойчивости схемы, а не к формальному определению 10.5. Лишь в одном случае будут описаны промежуточные коды $C_{1}, \ldots, C_{l-1}$ и разбиение схемы $\Sigma$ на ступени $\Sigma_{1}, \ldots, \Sigma_{l}$, как этого требует определение ступенчатой реализации.

Пусть $1 \ll s^{\prime} \ll s \ll k$, т.е. числа $s^{\prime}, s / s^{\prime}$ и $k / s$ достаточно велики. (Проанализировав все доказательство, можно приписать параметрам $s, s^{\prime}$ и $k$ конкретные значения.) В качестве основного кода $C$ (см. формулировку теоремы 10.7$)$ выбирается 
код $\operatorname{TOR}(k)$. Как и всякий симплектический код, он допускает кодирование и декодирование схемами полиномиального размера в базисе $\mathscr{S}$. Из двух информационных кубитов кода $\operatorname{TOR}(k)$ будет использоваться лишш один, например, соответствуюший информационным операторам $Y_{x 1}$ и $Y_{z 1}$.

Положим $\Gamma=\{B \subseteq \Delta:|B| \leqslant s\}, \Gamma^{\prime}=\left\{B \subseteq \Delta:|B| \leqslant s^{\prime}\right\}$, где $\Delta=\left\{1, \ldots, 2 k^{2}\right\}-$ множество кодовых кубитов. Тождественное преобразование $I_{\mathscr{B}}$ будет реализовано $\left(2,2 \Gamma, \Gamma^{\prime}\right)$-устойчивой схемой $\Sigma$. Все остальные элементы базиса будут реализованы $\left(2, \Gamma^{\prime}, \Gamma\right)$-устойчивьм образом. Чтобы сделать их $(2,2 \Gamma, \Gamma)$-устойчивыми, нужно предварительно уменьшить число ошибок на входе с помошью схемы $\Sigma$ (ср. с рис. $2 \mathrm{~b})$.

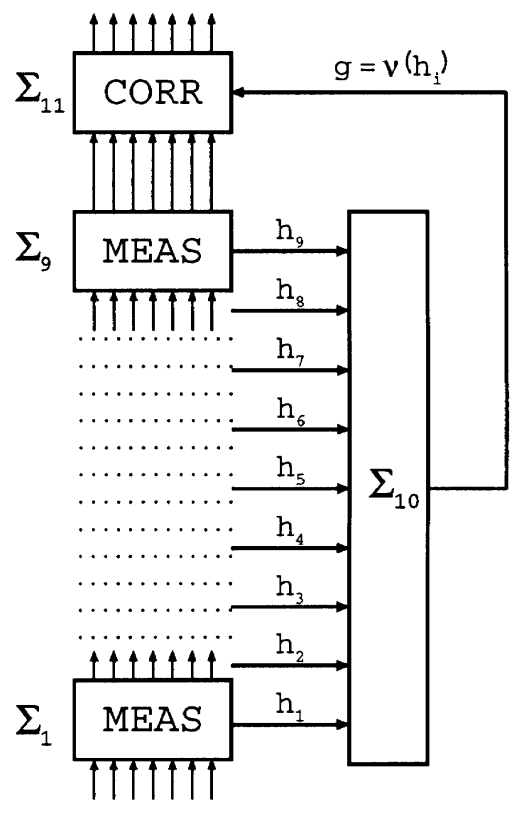

Рис. 3. (2, $\left.2 \Gamma, \Gamma^{\prime}\right)$-устойчивая ступенчатая реализация преобразования $I_{\mathscr{B}}$ в коде $\operatorname{TOR}(k)$

$\left(2,2 \Gamma, \Gamma^{\prime}\right)$-устойчивая ступенчатая реализация преобразования $I_{\mathscr{B}} \cdot(\mathrm{Cм}$. рис. 3.) Рассмотрим схему $\Sigma=\Sigma_{11} \circ \cdots \circ \Sigma_{1}$. Первые 9 ступеней $\left(\Sigma_{1}, \ldots, \Sigma_{9}\right)$ - это схемы MEAS (см. раздел 9.3), измеряюшие синдромы. Каждый из измеренных синдромов $h_{i}(i=1, \ldots, 9)$ записывается в отдельный регистр. Классическая схема $\Sigma_{10}$ находит такое $i$, что $h_{i}=h_{i+1}=h_{i+2}$ (существование такого $i$ будет доказано ниже), и вычисляет соответствуюшую ошибку $g=\nu\left(h_{i}\right)$. Схема $\Sigma_{11}=$ CORR исправляет эту ошибку.

$\left(2,2 \Gamma, \Gamma^{\prime}\right)$-устойчивость вытекает из следуюших соображений. Допустим, что при работе схемы $\Sigma$ случилось две ошибки. Всегда найдется такое $i$, что подсхемы $\Sigma_{i}$, $\Sigma_{i+1}, \Sigma_{i+2}$ сработали правильно. Следовательно, $h_{i}=h_{i+1}=h_{i+2}$. Обратно, пусть $h_{i}=h_{i+1}=h_{i+2}$ (такое $i$ может не быть единственным). Очевидно, что хотя бы одна из подсхем $\Sigma_{i}, \Sigma_{i+1}, \Sigma_{i+2}$ сработала без ошибок (поскольку ошибок всего две). Таким образом, $h_{i}$ является правильным синдромом, соответствуюшим $j$-му измерению $(i \leqslant j \leqslant i+2)$. Последующие измерения могут не только дать неправильные 
результаты, но и испортить некоторые из кодовых кубитов. Однако (это ключевое место во всей конструкции!) глубина схемы $\Sigma_{9} \circ \cdots \circ \Sigma_{1}$ ограничена, поскольку коды $\operatorname{TOR}(k)$ являются кодами с локальньми проверками. Следовательно, каждая из двух возможных ошибок может испортить лишь $O(1)$ кубитов. Таким образом, $2 s$ старых (сушествовавших до применения процедуры $\Sigma$ ) ошибок в кодовых кубитах будут исправлены, а новых возникнет всего $O(1) \leqslant s^{\prime}$. (Замечание: схема $\Sigma_{11}=$ CORR также имеет глубину $O(1)$, а классическая схема $\Sigma_{10}$ предполагается абсолютно надежной.)

Приведенные рассуждения можно сделать строгими, если предъявить промежуточные коды $C_{1}, \ldots, C_{10}$ из определения ступенчатой реализации. Пусть $n=2 k^{2}$ - число кодовых кубитов кода $C$. Определим комбинированное пространство $\mathscr{N}=$ $\mathscr{B} \otimes n \times G^{n}$ и пространство ошибок

$$
\mathscr{E}=(\sigma(g) \otimes|g\rangle: g \in E(n, 2 s)) \subseteq \mathbf{L}\left(\mathscr{B}^{\otimes n}\right) \otimes \bar{G}^{n}=\mathbf{L}\left(\mathscr{B}^{\otimes n}, \overline{\mathscr{N}}\right) .
$$

(Множество $E(n, 2 s)$ определено по формуле (52).) Положим $C_{10}=D=\operatorname{Der}(C, \mathscr{E})$. Иначе говоря, код $D$ получается из кода $C$ путем внесения произвольной ошибки $\sigma(g) \in$ $\mathbf{L}\left(\mathscr{B}^{\otimes n}\right)$ с носителем $\operatorname{Supp}(g) \in 2 \Gamma$, причем $g$ одновременно записывается в дополнительный классический регистр. Код $C_{i}(i=1, \ldots, 9)$ получается из кода $D$, если заменить содержимое $g$ классического регистра на $i$ копий синдрома $\mu(g)$. Используя эти коды, можно придать точньй смысл словам "правильньй синдромом, соответствующий $j$-му измерению” (см. предыдуший абзац). Построение множеств $B_{1}, \ldots, B_{11}$ из определения 10.5 остается читателю в качестве упражнения. Заметим также, что $\operatorname{Trans}(\Sigma)$ представляет $I_{\mathscr{B}}$ в коде $C$ в сильном смысле.

Соглашения. В дальнейшем под устойчивостью понимается $\left(2, \Gamma^{\prime}, \Gamma\right)$-устойчивость. Под реализацией унитарного оператора $U$ понимается реализация преобразования $U \cdot U^{\dagger}$.

Устойчивая реализация оператора $\widehat{\oplus}$. Пусть $A^{\prime}, A^{\prime \prime}$ - два множества кодовых кубитов, каждое из которых представляет свой информационньй кубит. Применим оператор (Ф) к каждой паре кодовых кубитов, один из которых принадлежит $A^{\prime}$, а другой - $A^{\prime \prime}$. При этом проверочные операторы преобразуются друг через друга, а информационные операторы $Y_{\alpha 1}^{\prime}=Y_{\alpha 1}\left[A^{\prime}\right], Y_{\alpha 1}^{\prime \prime}=Y_{\alpha 1}\left[A^{\prime \prime}\right]$ преобразуются по правилу

$$
Y_{x 1}^{\prime} \mapsto Y_{x 1}^{\prime} Y_{x 1}^{\prime \prime}, \quad Y_{x 1}^{\prime \prime} \mapsto Y_{x 1}^{\prime \prime}, \quad Y_{z 1}^{\prime} \mapsto Y_{z 1}^{\prime}, \quad Y_{z 1}^{\prime \prime} \mapsto Y_{z 1}^{\prime} Y_{z 1}^{\prime \prime}
$$

что и требуется. (Это общее свойство квазилинейных кодов.) Устойчивость этой схемы очевидна.

Устойчивая реализация оператора $S$. Применим оператор $S$ к каждому кодовому кубиту. Затем переставим кодовые кубиты, отразив квадратную сетку (см. рис. 1) относительно диагонали со сдвигом на $\frac{1}{2}$ периода. (При этом вершины перейдут в грани, грани в вершины, а ребра в ребра.) Информационные операторы $Y_{x 1}$ и $Y_{z 1}$ поменяются местами, что и требуется.

Устойчивая реализация преобразования $Q_{\mathbf{B}}$. Пусть $A_{1}$ - входной регистр, $A_{2}, \ldots, A_{5}$ - вспомогательные регистры. Занесем в них состояние $|0\rangle$, представленное в коде $C$. Скопируем основной регистр во вспомогательные, применяя операторы $\widehat{(\oplus)}\left[A_{1}, A_{i}\right](i=2,3,4,5)$. (В действительности, нужно использовать не сам оператор 
$\widehat{(\oplus), ~ а ~ о п и с а н н у ю ~ в ы ш е ~ у с т о и ̆ ч и в у ю ~ р е а л и з а ц и ю ~ э т о г о ~ о п е р а т о р а ~ в ~ к о д е ~} C$.) Применим к каждому из регистров $A_{1}, \ldots, A_{5}$ декодируюшее преобразование, а затем преобра-

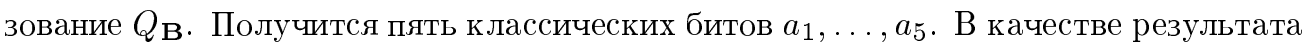
возьмем $\mathrm{MAJ}\left(a_{1}, \ldots, a_{5}\right)$. Легко видеть, что вся процедура устойчива к двум ошибкам.

Устойчивая реализация состояния $|0\rangle$. Представим состояние $|0\rangle$ в коде $C$. Сделаем копию (с помощью устойчивой реализации оператора (ิ)$)$. Применим к этой копии устойчивую реализацию преобразования $Q_{\mathbf{B}}$. Если получился 0 , то все в порядке, если единица - придется повторить все сначала. Если снова получилась единища - повторить в третий раз. (Этого всегда достаточно, так как мы предполагаем, что больше двух ошибок произойти не может.)

Устойчивая реализация оператора $K$. Решим сначала вспомогательную задачу: реализуем (устойчивым образом) состояния $\left|\eta_{ \pm}\right\rangle=2^{-1 / 2}(|0\rangle \pm \mathrm{i}|1\rangle)$. Эти состояния являются собственными для оператора $\sigma_{y}=-\mathrm{i} \sigma_{z} \sigma_{x}$. Задача будет решена, если мы сможем измерить собственные значения оператора $\sigma_{z} \sigma_{x}$, равные \pm i. Иными словами, требуется найти устойчивую реализацию преобразования

$$
P: \mathbf{L}(\mathscr{B}) \rightarrow \mathbf{L}(\mathscr{B}) \times \mathbf{B}: \quad \rho \mapsto\left(\Pi_{+} \rho \Pi_{+}, 0\right)+\left(\Pi_{-} \rho \Pi_{-}, 1\right)
$$

где $\Pi_{ \pm}-$проекторы на подпространства $\left(\left|\eta_{ \pm}\right\rangle\right)$.

Используя описанные выше процедуры, можно построить устойчивую реализацию оператора $W=\Lambda\left(\sigma_{z} \sigma_{x}\right)$

$$
W[1,2]=S[2] \widehat{\oplus}[1,2] S[2] \widehat{\oplus}[1,2] .
$$

Представим преобразование $P$ в виде (cp. с (31))

$$
P[1,2 ; 1]=R[2]\left(W \cdot W^{\dagger}\right)[2,1](S \cdot S)[2](|0\rangle\langle 0|)[2],
$$

где $R=Q_{\mathbf{B}}(S \cdot S)\left(K^{\dagger} \cdot K\right)$. Мы не располагаем пока устойчивой реализацией преобразования $R$. Однако, если преобразование $R$ реализовано неустойчивым образом, ошибка при его выполнении испортит лишь классический бит 2 , но не квантовьй бит 1 (представленньй в коде $C$ ). Итак, нам следует повторить измерение пять раз и взять функцию МАЈ от пяти полученных результатов.

Теперь заметим, что $W\left|\xi, \eta_{+}\right\rangle=K|\xi\rangle \otimes\left|\eta_{+}\right\rangle$(для любого $|\xi\rangle \in \mathscr{B}$ ). Таким образом, если во втором кубите приготовить состояние $\left|\eta_{+}\right\rangle$, то оператор $W$ действует на первьй кубит так же, как оператор $K$.

Элементы с классическим управлением. Мы уже построили устойчивые реализации $\Phi_{U}$ для всех базисных симплектических операторов $U \in S$. Чтобы реализовать преобразование $\Lambda_{c}(U)$, нужно использовать управляюший бит во всех элементах схемы $\Phi_{U}$. При этом мы выходим за пределы базиса, поскольку некоторые из элементов схемы будут иметь два управляющих бита (один из которых уже присутствовал в схеме $\left.\Phi_{U}\right)$. Это положение можно исправить, воспользовавшись тождестBOM

$$
\Lambda_{c}\left(\Lambda_{c}(U)\right)[1,2, A]=\Lambda_{c}(U)[3, A] \wedge[3 ; 1,2] .
$$


11.2. Случай полного базиса. По существу, нам осталось построить устойчивую реализацию элемента Тоффоли $\widehat{\wedge}_{\oplus}$. Как и в случае оператора $K$, задача решается в два этапа. Сначала строится состояние

$$
|\psi\rangle=\frac{1}{2}(|000\rangle+|010\rangle+|100\rangle+|111\rangle) .
$$

Описьваемая конструкция принадлежит Shor'y [43].

Устойчивая реализация состояния $|\psi\rangle$ в коде $C$. Состояния $\left|\psi_{+}\right\rangle=|\psi\rangle$ и $\left|\psi_{-}\right\rangle=\sigma_{x}[3]|\psi\rangle$ являются собственными для оператора $U:|a, b, c\rangle \mapsto(-1)^{a b+c}|a, b, c\rangle$. Для решения задачи достаточно измерить собственное значение этого оператора устойчивым образом. В самом деле, пусть $P \in \mathbf{T}\left(\mathscr{B}^{\otimes 3}, \mathscr{B}^{\otimes 3} \times \mathbf{B}\right)$ - измеряющее преобразование. Тогда

$$
P(|\xi, \xi, \xi\rangle\langle\xi, \xi, \xi|)=\frac{1}{2}\left(\left|\psi_{+}\right\rangle\left\langle\psi_{+}\right|, 0\right)+\frac{1}{2}\left(\left|\psi_{-}\right\rangle\left\langle\psi_{-}\right|, 1\right),
$$

где $|\xi\rangle=S|0\rangle$. Таким образом, если применить преобразование $P$ к состоянию $|\xi, \xi, \xi\rangle$, то с вероятностью $\frac{1}{2}$ получится $\left|\psi_{+}\right\rangle$(и мы достоверно будем знать об этом).

Легко видеть, что преобразование $U \cdot U^{\dagger}$ является симплектическим. Обозначим через $\Phi$ его устойчивую реализацию в коде $C$. Каждый из элементов $T_{q}$ схемы $\Phi$ заменим на $\Lambda\left(T_{q}\right)$, используя отдельный управляющий кубит. Полученную схему обозначим через $\Phi^{\prime}$. Преобразование $T^{\prime}=\operatorname{Trans}\left(\Phi^{\prime}\right)$ представляет $\Lambda(U) \cdot \Lambda(U)^{\dagger}$ в коде $D \times C^{3}$, где $D$ - код вида $|a\rangle \mapsto|a, \ldots, a\rangle$. (С одного управляющего кубита оператора $\Lambda(U)$ делается столько копий, сколько элементов в схеме $\Phi$.) Заметим, однако, что код $D$ не зашишает от ошибок вида $\sigma_{z}[j]$.

Измерение собственного значения оператора $U$ (представленного в коде $C$ ) производится с помошњю преобразования

$$
Q_{\mathbf{B}}[0] \quad(S \cdot S)[0] \quad R_{\leftarrow}\left[0 ; A_{0}\right] \quad T^{\prime}\left[A_{0}, A_{1}, A_{2}, A_{3}\right] \quad(|\xi\rangle\langle\xi|)\left[A_{0}\right] .
$$

Здесь $R_{\leftarrow}$ - декодирующее преобразование для кода $D$, а $(|\xi\rangle\langle\xi|)\left[A_{0}\right]-$ это состояние $|\xi\rangle=S|0\rangle$, представленное в коде $D$ и записанное в регистр $A_{0}$. Его можно приготовить, например, таким образом: взять состояние $|\xi\rangle$ в коде $C$, размножить с помошю оператора $\widehat{\oplus}$ (получится $|\xi\rangle$ в коде $D \triangleleft C$ ), а затем декодировать каждую из полученных "копий". Схема (64) почти устойчива: две ошибки могут испортить лишь классический бит 0. Таким образом, следует повторить измерение пять раз и взять функцию МАJ.

В дальнейшем вместо состояния $|\psi\rangle$ мы будем использовать состояние

$$
|\zeta\rangle=S[3]|\psi\rangle=2^{-3 / 2} \sum_{a, b, c}(-1)^{a b c}|a, b, c\rangle .
$$

Заметим также, что $\widehat{\wedge}_{\oplus}=S[3] W S[3]$, где $W:|a, b, c\rangle \mapsto(-1)^{a b c}|a, b, c\rangle$.

Устойчивая реализация преобразования $W \cdot W^{\dagger}$. Рассмотрим унитарное вложение $V: \mathscr{B} \otimes 3 \rightarrow \mathscr{B} \otimes 6$

$$
V[1,2,3,4,5,6 ; 1,2,3]=\widehat{\oplus}[1,4] \widehat{\oplus}[2,5] \widehat{\oplus}[3,6](|\zeta\rangle[4,5,6]) .
$$


Применим преобразование $V \cdot V^{\dagger}$ к произвольному состоянию $\rho \in \mathbf{D}\left(\mathscr{B}{ }^{\otimes 3}\right)$ и измерим значения трех последних битов (с номерами $4,5,6)$. Эта процедура описьвается преобразованием $T \in \mathbf{T}\left(\mathscr{B}^{\otimes 3}, \mathscr{B}^{\otimes 3} \times \mathbf{B}^{3}\right)$ :

$$
T: \rho \mapsto \frac{1}{8} \sum_{\alpha, \beta, \gamma}\left(W_{\alpha \beta \gamma} \rho W_{\alpha \beta \gamma}^{\dagger}, \alpha, \beta, \gamma\right),
$$

где

$$
W_{\alpha \beta \gamma}:|a, b, c\rangle \mapsto(-1)^{(a+\alpha)(b+\beta)(c+\gamma)}|a, b, c\rangle .
$$

Теперь, чтобы получить нужное нам состояние $W \rho W^{\dagger}$, остается применить один из операторов $Y_{\alpha \beta \gamma}=W W_{\alpha \beta \gamma}^{-1}$ (в зависимости от результата измерения $\left.(\alpha, \beta, \gamma)\right)$. Легко проверить, что преобразования $Y_{\alpha \beta \gamma} \cdot Y_{\alpha \beta \gamma}^{\dagger}$ симплектичны, поэтому их можно реализовать устойчивым образом.

Автор признателен М. Вялому за многочисленные обсуждения и замечание о связи торических кодов с задачей о паросочетании наименьшего веса. Другое ценное замечание, сделанное С.П. Новиковым, позволило более компактно изложить теорию симплектических кодов. Автор благодарит также С. Тарасова за интересную дискуссию.

Работа вьполнена при поддержке Российского фонда фундаментальных исследований (грант № 96-01-01113).

\section{СПИСОК ЛИТЕРАТУРЫ}

[1] Манин Ю. И. Вычислимое и невычислимое. М.: Советское радио, 1980.

[2] Benioff P. Quantum mechanical Hamiltonian models of Turing machines // J. Statist. Phys. 1982. V. 29. № 3. P. 515-546.

[3] Peres A. Reversible logic and quantum computers // Phys. Rev. Ser. A. 1985. V. 32. № 6. P. 3266-3276.

[4] Feynman R.P. Quantum mechanical computers // Optics News. February 1985. V. 11. P. 11.

[5] Deutsch D. Quantum theory, the Church-Turing principle and the universal quantum computer // Proc. Roy. Soc. London. Ser. A. 1985. V. 400. № 1818. P. 97-117.

[6] Deutsch D. Quantum computational networks // Proc. Roy. Soc. London. Ser. A. 1989. V. 425. № 1868. P. 73-90.

[7] Yao A. C.-C. Quantum circuit complexity // Proceedings of the 34th Annual Symposium on the Foundations of Computer Science. Los Alamitos, CA: IEEE Comput. Soc. Press, 1993. P. $352-361$.

[8] Гэри М., Джонсон Д. Вычислительные машины и труднорешаемые задачи. М.: Мир, 1982.

[9] Turchette Q.A., Hood C. J., Lange W., Mabushi H., Kimble H. J. Measurement of conditional phase shifts for quantum logic // Phys. Rev. Lett. 1995. V. 75. № 25. P. 4710-4713.

[10] Monroe C., Meekhof D. M., King B. E., Itano W. M., Wineland D. J. Demonstration of a fundamental quantum logic gate // Phys. Rev. Lett. 1995 . V. 75 . № 25 . P. 4714-4717.

[11] Shor P. W. Algorithms for quantum computation: discretelog and factoring // Proceedings of the 35th Annual Symposium on the Foundations of Computer Science. Los Alamitos, CA: IEEE Computer Society Press, 1994. P. 124.

[12] Диффи У., Хэлманн М. Э. Защищенность и имитостойкость. Введение в криптографиюю // ТИИЭР. 1979. Т. 67. №3. 
[13] Simon D. On the power of quantum computation // Proceedings of the 35th Annual Symposium on the Foundations of Computer Science. Los Alamitos, CA: IEEE Computer Society Press, 1994. P. 116.

[14] Grigoriev D. Testing shift equivalence of polynomials using quantum machines // Preprint, 1995.

[15] Kitaev A. Yu. Quantum measurements and the Abelian stabilizer problem // LANL e-print quant-ph/9511026, http://xxx.lanl.gov.

[16] Bernstein E., Vazirani U. Quantum complexity theory // Proceedings of the 25th Annual ACM Symposium on Theory of Computing. New York: ACM Press, 1993. P. 11-20.

[17] Мак-Вильямс Ф. Дж., Слоэн Н. Дж. А. Теория кодов, исправляющих ошибки. М.: Связь, 1979.

[18] Shor P.W. Scheme for reducing decoherence in quantum computer memory // Phys. Rev. Ser. A. 1995. V. 52. № 4. P. 2493-2496.

[19] Calderbank A.R., Shor P.W. Good quantum error-correcting codes exist // LANL e-print quant-ph/9512032, http://xxx.lanl.gov (to appear in Phys. Rev. Ser. A).

[20] Steane A. M. Multiple-particle interference and quantum error correction // Proc. Roy. Soc. London. Ser. A. 1996. V. 1954. P. 2551-2577; // LANL e-print quant-ph/9601029, http://xxx.lanl.gov.

[21] Laflamme R., Miquel C., Paz J. P., Zurek W. H. Perfect quantum error correction code // LANL e-print quant-ph/9602019, http://xxx.lanl.gov.

[22] Plenio B. B., Vedral V., Knight P. L. Optimal realistic quantum error correcting code // LANL e-print quant-ph/9603022, http://xxx.lanl.gov.

[23] Vaidman L., Goldenberg L., Wiesner S. Error prevention scheme with four particles // LANL e-print quant-ph/9603031, http://xxx.lanl.gov.

[24] Shor P. W., Smolin J. A. Quantum error-correcting codes need not completely reveal the error syndrome // LANL e-print quant-ph/9604006, http://xxx.lanl.gov.

[25] Bennett C.H., DiVincenzo D.P., Smolin J.A., Wootters W. K. Mixed state entanglement and quantum error-correcting codes // LANL e-print quant-ph/9604024, http://xxx.lanl.gov.

[26] Braunstein S. L. Perfect quantum error correction coding in 26 laser pulses // LANL e-print quant-ph/9604036, http://xxx.lanl.gov.

[27] Gottesman D. A class of quantum error-correcting codes saturating the quantum Hamming bound // LANL e-print quant-ph/9604038, http://xxx.lanl.gov.

[28] Gottesman D. Stabilizer codes and quantum error correction // LANL e-print quant-ph/ $9705052 \mathrm{http} / /$ xxx.lanl.gov.

[29] Calderbank A.R., Rains E. M., Shor P. W., Sloane N. J. A. Quantum error correction and orthogonal geometry // LANL e-print quant-ph/9605005, http://xxx.lanl.gov.

[30] Rains E. M., Hardin R.H., Shor P. W., Sloane N. J. A. A nonadditive quantum code // LANL e-print quant-ph/9703002, http://xxx.lanl.gov.

[31] Холево А.С. Некоторые оценки для количества информации, передаваемого квантовым каналом связи // Проблемы передачи информации. 1973. Т. 9. №3. С. 3-11.

[32] Schumacher B. Sending quantum entanglement through noisy channels // LANL e-print quant-ph/9604023, http://xxx.lanl.gov.

[33] Schumacher B., Nielsen M. A. Quantum data processing and error correction // LANL e-print quant-ph/9604022, http://xxx.lanl.gov.

[34] Lloyd S. The capacity of the noisy quantum channel // LANL e-print quant-ph/9604015, http://xxx.lanl.gov.

[35] Ekert A., Macchiavello C. Error correction in quantum communication // LANL e-print quant-ph/9602022, http://xxx.lanl.gov.

[36] Knill E., Laflamme R. A theory of quantum error-correcting codes // LANL e-print quant-ph/9604034, http://xxx.lanl.gov.

[37] Shor P. W., Laflamme R. Quantum analog of the MacWilliams identities in classical coding theory // LANL e-print quant-ph/9610040, http://xxx.lanl.gov.

[38] Rains E. M. Quantum weight enumerators // LANL e-print quant-ph/9612015, http:// xxx.lanl.gov. 
[39] Bennett C. H., Brassard G., Crépeau C., Jozza R., Peres A., Wootters W. K. // Phys. Rev. Lett. 1993. V. 70. P. 1895.

[40] Bennett C.H., Brassard G., Popescu S., Schumacher B., Smolin J.A., Wootters W. K. Purification of noisy entanglement and faithful teleportation via noisy channels // Phys. Rev. Lett. 1996. V. 76. № 5. P. 722-725.

[41] Bennett C.H., DiVincenzo D.P., Smolin J.A., Wootters W. K. Mixed state entanglement and quantum error correction // Phys. Rev. Ser. A. 1996. V. 54 . № 5 . P. 3824-3851; // LANL e-print quant-ph/9604024, http://xxx.lanl.gov.

[42] Kitaev A. Yu. Quantum error correction with imperfect gates // Quantum Communication, Computing and Measurement / ed. O. Hirota, A.S. Holevo, C. M. Caves. New York: Plenum Press, 1997.

[43] Shor P.W. Fault-tolerant quantum computation // LANL e-print quant-ph/9605011, http://xxx.lanl.gov.

[44] Knill E., Laflamme R. Concatenated quantum codes // LANL e-print quant-ph/9608012, http://xxx.lanl.gov.

[45] Knill E., Laflamme R., Zurek W. Accuracy threshold for quantum computation // LANL e-print quant-ph/9610011, http://xxx.lanl.gov.

[46] Aharonov D., Ben-Or M. Fault tolerant quantum computation with constant error // LANL e-print quant-ph/9611025, http://xxx.lanl.gov.

[47] Gottesman D. A theory of fault-tolerant quantum computation // LANL e-print quant-ph/9702029, http://xxx.lanl.gov.

[48] Kitaev A. Yu. Fault-tolerant quantum computation by anyons // LANL e-print quant-ph/9707021, http://xxx.lanl.gov.

[49] Miller G. L. Riemann's hypothesis and tests for primarity // J. Comput. System Sci. 1976. V. 13. P. $300-317$.

[50] Lecerf Y. Machines de Turing reversibles. Recursive insolubilité en $n \in N$ de l'equation $u=\theta^{n}$ où $\theta$ est un "isomorphisme de codes" // C. R. Acad. Sci. Paris. 1963. V. 257. P. 2597-2600.

[51] Bennett C. H. Logical reversibility of computation // IBM J. Res. Develop. 1973. V. 17. P. 525-532.

[52] Landauer R. Irreversibility and heat generation in the computing process // IBM J. Res. Develop. 1961. V. 5. P. 183.

[53] Barenco A., Bennett C.H., Cleve R., DiVincenzo D.P., Margolus N., Shor P., Sleator T., Smolin J., Weinfurter H. Elementary gates for quantum computation // quant-ph/9503016.

[54] Solovay Robert. Частное сообщение.

[55] Пападимитриу Х., Стайглитц К. Комбинаторная оптимизация: алгоритмы и сложность. М.: Мир, 1985.

Институт теоретической физики

Поступила в редакцию

им. Л. Д. Ландау

28.12 .1996

E-mail: kitaev@itp.ac.ru 